\title{
Front Matter: Volume 10646
}

, "Front Matter: Volume 10646," Proc. SPIE 10646, Signal Processing, Sensor/Information Fusion, and Target Recognition XXVII, 1064601 (12 June 2018); doi: $10.1117 / 12.2500434$

SPIE. Event: SPIE Defense + Security, 2018, Orlando, FL, United States 


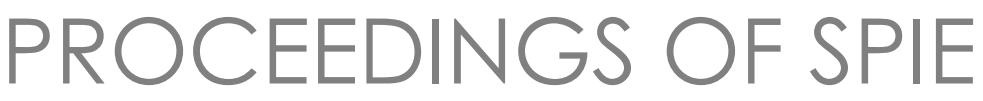

\title{
Signal Processing, Sensor/Information Fusion, and Target Recognition XXVII
}

\author{
Ivan Kadar \\ Editor
}

16-19 April 2018

Orlando, Florida, United States

Sponsored and Published by

SPIE 
The papers in this volume were part of the technical conference cited on the cover and title page. Papers were selected and subject to review by the editors and conference program committee. Some conference presentations may not be available for publication. Additional papers and presentation recordings may be available online in the SPIE Digital Library at SPIEDigitalLibrary.org.

The papers reflect the work and thoughts of the authors and are published herein as submitted. The publisher is not responsible for the validity of the information or for any outcomes resulting from reliance thereon.

Please use the following format to cite material from these proceedings:

Author(s), "Title of Paper," in Signal Processing, Sensor/Information Fusion, and Target Recognition XXVII, edited by Ivan Kadar, Proceedings of SPIE Vol. 10646 (SPIE, Bellingham, WA, 2018) Seven-digit Article CID Number.

ISSN: 0277-786X

ISSN: 1996-756X (electronic)

ISBN: 9781510618039

ISBN: 9781510618046 (electronic)

Published by

SPIE

P.O. Box 10, Bellingham, Washington 98227-0010 USA

Telephone +1 3606763290 (Pacific Time) · Fax +1360647 1445

SPIE.org

Copyright $@ 2018$, Society of Photo-Optical Instrumentation Engineers.

Copying of material in this book for internal or personal use, or for the internal or personal use of specific clients, beyond the fair use provisions granted by the U.S. Copyright Law is authorized by SPIE subject to payment of copying fees. The Transactional Reporting Service base fee for this volume is $\$ 18.00$ per article (or portion thereof), which should be paid directly to the Copyright Clearance Center (CCC), 222 Rosewood Drive, Danvers, MA 01923. Payment may also be made electronically through CCC Online at copyright.com. Other copying for republication, resale, advertising or promotion, or any form of systematic or multiple reproduction of any material in this book is prohibited except with permission in writing from the publisher. The CCC fee code is 0277 $786 \mathrm{X} / 18 / \$ 18.00$.

Printed in the United States of America.

Publication of record for individual papers is online in the SPIE Digital Library.

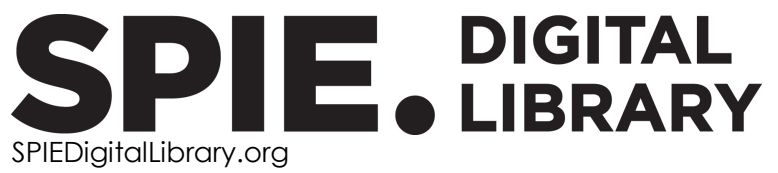

Paper Numbering: Proceedings of SPIE follow an e-First publication model. A unique citation identifier (CID) number is assigned to each article at the time of publication. Utilization of CIDs allows articles to be fully citable as soon as they are published online, and connects the same identifier to all online and print versions of the publication. SPIE uses a seven-digit CID article numbering system structured as follows:

- The first five digits correspond to the SPIE volume number.

- The last two digits indicate publication order within the volume using a Base 36 numbering system employing both numerals and letters. These two-number sets start with 00, 01, 02, 03, 04, $05,06,07,08,09,0 A, 0 B \ldots$. OZ, followed by 10-1Z, 20-2Z, etc. The CID Number appears on each page of the manuscript. 


\title{
Contents
}

\author{
vii $\quad$ Authors \\ ix Conference Committee \\ xiii Introduction to the Invited Panel Discussion \\ xv Invited Panel Slides
}

SESSION 1 MULTISENSOR FUSION, MULTITARGET TRACKING, AND RESOURCE MANAGEMENT I

1064602 Track stitching and approximate track association on a pairwise-likelihood graph [10646-1]

1064603 From labels to tracks: it's complicated [10646-2]

1064604 An introduction to the generalized labeled multi-Bernoulli filter through Matlab code [10646-3]

1064605 On-orbit calibration of satellite based imaging sensors [10646-5]

1064606 The data-driven 8 -generalized labeled multi-Bernoulli tracker for automatic birth initialization [10646-4]

SESSION 2 MULTISENSOR FUSION, MULTITARGET TRACKING, AND RESOURCE MANAGEMENT II

1064607 Localization of a point target from an optical sensor's focal plane array [10646-6]

1064609 Trajectory estimation and impact point prediction of a ballistic object from a single fixed passive sensor [10646-8]

10646 OA A study of particle filtering approaches for the kidnapped robot problem [10646-9]

10646 OB Evaluation of optimizations of Murty's M-best assignment [10646-10]

SESSION $3 \quad$ INFORMATION FUSION METHODOLOGIES AND APPLICATIONS I

10646 OC A generalized labeled multi-Bernoulli filter for correlated multitarget systems [10646-11]

10646 OD A clutter-agnostic generalized labeled multi-Bernoulli filter [10646-12] 
10646 OE A fast labeled multi-Bernoulli filter for superpositional sensors [10646-13]

10646 OF Large constellation tracking using a labeled multi-Bernoulli filter [10646-14]

SESSION 4 INFORMATION FUSION METHODOLOGIES AND APPLICATIONS II

10646 OG Detection system fusion based on the predictive value curve and its variations [10646-15]

$10646 \mathrm{OH} \quad$ Improving ATR system performance through sequences of classification tasks [10646-16]

SESSION 5 INFORMATION FUSION METHODOLOGIES AND APPLICATIONS III

10646 OK pystemlib: towards an open-source tracking, state estimation, and mapping toolbox in Python [10646-19]

$10646 \mathrm{OL} \quad$ Analysis of noise impact on distributed average consensus [10646-20]

SESSION 6 INFORMATION FUSION METHODOLOGIES AND APPLICATIONS IV

10646 OM A framework for adaptive MaxEnt modeling within distributed sensors and decision fusion for robust target detection/recognition [10646-21]

10646 ON A comparison between robust information theoretic estimator and asymptotic maximum likelihood estimator for misspecified model [10646-22]

1064600 Optimizing collaborative computations for scalable distributed inference in large graphs [10646-23]

10646 OP Enabling self-configuration of fusion networks via scalable opportunistic sensor calibration [10646-24]

$106460 Q \quad$ Multiscale graph-based framework for efficient multi-sensor integration and event detection [10646-25]

SESSION 7 INFORMATION FUSION METHODOLOGIES AND APPLICATIONS V

10646 OS An adaptive sensing approach for the detection of small UAV: first investigation of static sensor network and moving sensor platform [10646-27]

10646 OU MARINE-EO bridging innovative downstream earth observation and Copernicus enabled services for integrated maritime environment, surveillance, and security [10646-29]

10646 OV A novel architecture for behavior/event detection in security and safety management systems [10646-30]

iv 
10646 OW A new FSII-CFAR detector based on fuzzy membership degree [10646-31]

$106460 Z$ Low-cost multi-camera module and processor array for the ultra-fast framerate recognition, location, and characterization of explosive events [10646-34]

1064610 Super-resolution of remote sensing images using edge-directed radial basis functions [10646-35]

1064611 Compressive sensing using the log-barrier algorithm with complex-valued measurements [10646-36]

\section{SESSION 9 SIGNAL AND IMAGE PROCESSING, AND INFORMATION FUSION APPLICATIONS II}

1064612 Stabilization and registration of full-motion video data using deep convolutional neural networks [10646-37]

1064613 iSight: computer vision based system to assist low vision [10646-38]

1064614 A real-time object detection framework for aerial imagery using deep neural networks and synthetic training images [10646-39]

$1064615 \quad$ Mobile crowd-sensing for access point localization [10646-40]

1064616 Going deeper with CNN in malicious crowd event classification [10646-41]

SESSION 10 SIGNAL AND IMAGE PROCESSING, AND INFORMATION FUSION APPLICATIONS III

10646 1A Scanning LiDAR for airfield damage assessment [10646-47]

\section{SESSION 11 SIGNAL AND DATA PROCESSING FOR SMALL TARGETS}

10646 IF Robust spectral classification [10646-50]

$106461 \mathrm{H}$ Separation of small targets in multi-wavelength mixtures based on statistical independence [10646-52]

$106461 \mathrm{~K} \quad$ Error statistics of bias-naïve filtering in the presence of bias [10646-55]

$10646 \mathrm{lL} \quad$ An analytic solution to ellipsoid intersections for multistatic radar [10646-56] 
$106461 \mathrm{M} \quad$ Multilevel probabilistic target identification methodology utilizing multiple heterogeneous sensors providing various levels of target characteristics [10646-57]

\section{POSTER SESSION}

10646 IP Attitude control system for a balloon based telescope [10646-60]

10646 IR Blind modulation detection via staged GLRT [10646-62]

10646 IS CRLB for estimation of 3D sensor biases in spherical coordinates [10646-63] 


\section{Authors}

Numbers in the index correspond to the last two digits of the seven-digit citation identifier (CID) article numbering system used in Proceedings of SPIE. The first five digits reflect the volume number. Base 36 numbering is employed for the last two digits and indicates the order of articles within the volume. Numbers start with 00, 01, 02, 03, 04, 05, 06, 07, 08, 09, OA, OB...0Z, followed by 10-12, 20-2Z, etc.

\author{
Altman, Emilie, OK \\ Anderson, Evan, $1 \mathrm{~K}$ \\ Angotta, Bill, 1A \\ Astyakopoulos, Alkis, OU \\ Bacher, E., OS \\ Bailey, Colleen P., IR \\ Balaji, Bhashyam, OK \\ Balasingam, Balakumar, 07 \\ Bar-Shalom, Yaakov, 05, 07, 09, 0B, is \\ Bayer, Michael A., 12 \\ Belfadel, Djedjiga, 05, is \\ Ben-Dov, R., 09 \\ Borel-Donohue, Christoph, 14 \\ Bothos, John, OV \\ Carniglia, Peter, OK \\ Chance, Zachary, $1 \mathrm{~K}$ \\ Chandran, Krishnan, 13 \\ Chen, Lingji, 02, 03, 04 \\ Colonna-Romano, John, 00 \\ Copsey, Keith, OP \\ Couwenhoven, Doug W., 12 \\ Cox, Kevin, $\mathrm{OZ}$ \\ da Silva, Felipe B., 11 \\ DeMars, Kyle J., 06 \\ Diltz, Robert, $1 \mathrm{~A}$ \\ Dou, Wenbo, OB \\ Dunham, Joel, $1 \mathrm{M}$ \\ Emge, Darren K., $1 \mathrm{H}$ \\ English, Woody, $1 \mathrm{~A}$ \\ Eum, Sungmin, 16 \\ Fowler, Stuart, 1A \\ Gatsak, Tatiana, OK \\ Giovanneschi, F., OS \\ Gómez Miguel, Beatriz, OU \\ Grewe, Lynne, 13 \\ Hammer, M., OS \\ Hengy, S., OS \\ Hommes, A., OS \\ Hurley, Jeffery D., 1M \\ Johannes, W., OS \\ Johnson, Clint, $1 \mathrm{M}$ \\ Jones, Brandon A., OF \\ Kadar, Ivan, OM \\ Kashyap, Archana, 13 \\ Kay, Steven M., ON, IF \\ Kong, Yingying, OW \\ Kontoes, Haris, OU \\ Kowalski, Michael, is \\ Kwon, Heesung, 14, 16
}

Kyriazanos, Dimitris M., OU, OV

Langford, Darrell, 1A

Laurenzis, M., OS

Lee, Hyungtae, 14, 16

LeGrand, Keith A., 06

Leirens, Sylvain, 15

Le Moigne, Jaqueline, 10

Leung, Henry, OL, OW

Levchuk, Georgiy, 00

Li, Boyuan, OL

Lu, Qin, 07, OB

Lykousis, Vasilios, OU

Mahler, Ronald, OC, OD, OE

Maraviglia, Carlos, $\mathrm{OZ}$

Mareboyana, Manohar, 10

Markellou, Marina, OU

Martins, Ana, OU

McArdle, Sean M., OF

Milgrom, B., 09

Miosso, Cristiano J., 11

Mohler, David, OA

Mowakeaa, Rami, $1 \mathrm{H}$

Mulgrew, Bernard, OP

Narayanan, Priya, 14

Nehmetallah, George, 1P

Oxley, Mark E., OG, OH

Pados, Dimitris A., IR

Page, Scott, OP

Park, Sungjoo, $O Z$

Pattipati, Krishna, OB

Pereira da Silva, Alex, 15

Poyet, J.-M., OS

Prasad, Lakshman, $\mathrm{OQ}$

Rao, Raghuveer, 14

Rassy, O., OS

Ravago, Nicholas, OF

Relyea, Stephen, $1 \mathrm{~K}$

Rinehart, Stephen A., IP

Rizogiannis, Constantinos, OU, OV

Robinson, Brian, $1 \mathrm{~A}$

Rumbley, Sarah E., 02

Schertzer, S., OS

Schubert Kabban, Christine M., OG, $\mathrm{OH}$

Seneviratne, Chatura, OL

Shah, Akhil K., OF

Shahshahani, Allen, 13

Shahshahani, Jake, 13

Shapero, Samuel A., IL

Simmons, Jimmy, $1 M$ 
Taylor, Clark N., OA

Thanos, Konstantinos Georgios, OV

Thomas, Paul, OP

Thomopoulos, Stelios C. A., OU, OV

Trypitsidis, Anestis, OU

Tsouni, Alexia, OU

Tucker, Andrew W., IF

Üney, Murat, OP

Varkitzi, loanna, OU

Vieira, Fábio AL., OU

Vila Hernandez de Lorenzo, Jordi, IP

Visina, Radu, OB

von Borries, Ricardo, 11

Walters, Josh, 1A

Walvoord, Derek J., 12

Willett, Peter, 07, 09, 0B, $1 \mathrm{~S}$

Yang, Kaipei, 09

Yoedt, Cedric, $0 Z$

Zalonis, Andreas, OV

Zhang, Shu, OW

Zhou, Xin, ON 


\section{Conference Committee}

Symposium Chair

Arthur A. Morrish, Raytheon Space and Airborne Systems

(United States)

Symposium Co-chair

Ruth L. Moser, Air Force Research Laboratory (United States)

Conference Chairs

Ivan Kadar, Interlink Systems Sciences, Inc. (United States)

Conference Co-chairs

Bhashyam Balaji, Defence Research and Development Canada (Canada)

Erik P. Blasch, Air Force Research Laboratory (United States)

Lynne L. Grewe, California State University, East Bay (United States)

Thia Kirubarajan, McMaster University (Canada)

Ronald P. S. Mahler, Random Sets, LLC (United States)

Conference Program Committee

Mark G. Alford, Air Force Research Laboratory (United States)

William D. Blair, Georgia Tech Research Institute (United States)

Mark J. Carlotto, General Dynamics Advanced Information Systems

(United States)

Alex L. Chan, U.S. Army Research Laboratory (United States)

Kuo-Chu Chang, George Mason University (United States)

Chee-Yee Chong, Independent Consultant (United States)

Marvin N. Cohen, Georgia Tech Research Institute (United States)

Frederick E. Daum, Raytheon Company (United States)

Jean Dezert, Office National d'Etudes et de Recherches

Aérospatiales (France)

Mohammad Farooq, AA Scientific Consultants Inc. (Canada)

Laurie H. Fenstermacher, Air Force Research Laboratory

(United States)

Charles W. Glover, Oak Ridge National Laboratory (United States)

I. R. Goodman, Consultant (United States)

Michael L. Hinman, Independent Consultant (United States)

Jon S. Jones, Air Force Research Laboratory (United States)

Georgiy M. Levchuk, Aptima, Inc. (United States) 
Martin E. Liggins II, Independent Consultant (United States)

James Llinas, University at Buffalo (United States)

Raj P. Malhotra, Air Force Research Laboratory (United States)

Alastair D. McAulay, Lehigh University (United States)

Raman K. Mehra, Scientific Systems Company, Inc. (United States)

Harley R. Myler, Lamar University (United States)

David Nicholson, BAE Systems (United Kingdom)

Les Novak, Scientific Systems Company, Inc. (United States)

John J. Salerno Jr., Harris Corporation (United States)

Robert W. Schutz, Consultant (United States)

Andrew G. Tescher, AGT Associates (United States)

Stelios C. A. Thomopoulos, National Center for Scientific Research

Demokritos (Greece)

Wiley E. Thompson, New Mexico State University (United States)

Shanchieh Jay Yang, Rochester Institute of Technology (United States)

\section{Session Chairs}

1 Multisensor Fusion, Multitarget Tracking, and Resource Management I

Ivan Kadar, Interlink Systems Sciences, Inc. (United States)

2 Multisensor Fusion, Multitarget Tracking, and Resource Management II

Ivan Kadar, Interlink Systems Sciences, Inc. (United States)

3 Information Fusion Methodologies and Applications I

Ronald P. S. Mahler, Random Sets LLC (United States)

4 Information Fusion Methodologies and Applications II Ivan Kadar, Interlink Systems Sciences, Inc. (United States) Chee-Yee Chong, Independent Consultant (United States)

5 Information Fusion Methodologies and Applications III Chee-Yee Chong, Independent Consultant (United States) Ivan Kadar, Interlink Systems Sciences, Inc. (United States)

6 Information Fusion Methodologies and Applications IV Chee-Yee Chong, Independent Consultant (United States) Ivan Kadar, Interlink Systems Sciences, Inc. (United States)

7 Information Fusion Methodologies and Applications $V$ Erik Blasch, Air Force Research Laboratory (United States) Ivan Kadar, Interlink Systems Sciences, Inc. (United States) Chee-Yee Chong, Independent Consultant (United States) 
8 Signal and Image Processing, and Information Fusion Applications I Lynne L. Grewe, California State University, East Bay (United States) Alex L. Chan, U.S. Army Research Laboratory (United States)

9 Signal and Image Processing, and Information Fusion Applications II Lynne L. Grewe, California State University, East Bay (United States) Alex L. Chan, U.S. Army Research Laboratory (United States)

10 Signal and Image Processing, and Information Fusion Applications III Alex L. Chan, U.S. Army Research Laboratory (United States) Lynne L. Grewe, California State University, East Bay (United States)

11 Signal and Data Processing for Small Targets

Philip D. West, Georgia Tech Research Institute (United States)

Darren K. Emge, U.S. Army Edgewood Chemical Biological Center (United States) 
Proc. of SPIE Vol. 10646 1064601-12 Downloaded From: https://www.spiedigitallibrary.org/conference-proceedings-of-spie on 26 Apr 2023
Terms of Use: https://www.spiedigitallibrary.org/terms-of-use 


\section{Introduction to the Invited Panel Discussion}

\section{Deep Learning in Al and Information Fusion}

In the early days of artificial intelligence (Al) starting, say in the 1970s and 1980s, the predominant reasoning methods were logical and symbolic, using, e.g., Lisp/Prolog languages. Later in the 1980s, Al tools were used such as Knowledge Environment Engineering (KEE) and Automated Reasoning Tool (ART) expert systems, and early heuristic reasoning methods. Also, the concept and mathematical representation of "context" logic was defined. The concept and apps of both "knowledge based" and "context" are currently used in several apps in information fusion (IF) along with several methods to apply and learn contextual information.

In the early 1980's, Al was viewed as the solution to information fusion problems. In fact, many contributors to the first distributed sensor networks program were Al researchers. However, inadequate computing and $\mathrm{Al}$ approaches such as expert systems and heuristic uncertainty reasoning could not address the challenges of information fusion. Thus, important advances in information fusion, and in particular, multi-target tracking, were made with little contribution from Al.

During the long Al winter, researchers addressed the deficiencies of early Al, developing rigorous representation and reasoning techniques for uncertainty, and machine learning approaches. Recently, data science was established as a popular area to exploit the large volumes of data (a.k.a. Big Data) collected by physical sensors and online activities using machine learning and other analytic tools.

Artificial intelligence and data science pose both challenges and opportunities to IF. They are challenges because they appear to address the same problems as information fusion, but with more powerful techniques, thus siphoning away both research funding and research talent. However, these challenges can also be opportunities because Al and data science provide new research directions for information fusion. Examples include: IF with big data, hard and soft data fusion, learning about context, graph techniques for tracking and fusion, dynamic network analysis, apps to cyber and imagery processing.

The objective of this panel was to bring to the attention of the fusion community the importance of the application of deep learning in AI and IF, highlighting issues, illustrating approaches and addressing challenges. A number of invited experts discussed challenges in processing and research, and addressed these challenges with IF. The panelists illustrated parts of the abovementioned areas over different applications and association with IF. The panel highlighted impending issues and challenges using conceptual and real-world related examples associated with the applications of above.

Chee-Yee Chong Ivan Kadar 
Proc. of SPIE Vol. 10646 1064601-14 Downloaded From: https://www.spiedigitallibrary.org/conference-proceedings-of-spie on 26 Apr 2023
Terms of Use: https://www.spiedigitallibrary.org/terms-of-use 


\section{Invited Panel Discussion Deep Learning in Al and Information Fusion}

\section{Organizers}

Chee-Yee Chong, Independent Consultant

Ivan Kadar, Interlink Systems Sciences, Inc.

Erik Blasch, Air Force Research Lab

\section{Moderators}

Ivan Kadar, Interlink Systems Sciences, Inc.

Chee-Yee Chong, Independent Consultant

April 16, 2018

SPIE Conference 10646

"Signal Processing, Sensor Fusion and Target Recognition XXVII"

Orlando, ,FL 16-19 April 2018

\section{Invited Panel Discussion}

Panel Participants:

Dr. Erik Blasch, Air Force Research Lab., U.S.A.

Dr. Chee-Yee Chong, Independent Consultant, U.S.A.

Professor George Cybenko, Dartmouth College, NH, U.S.A

(unable to attend

Professor Lynne Grewe, California State Univ, East Bay, U.S.A

Dr. Ivan Kadar, Interlink Systems Sciences, Inc., U.S.A.

Dr. Uttam K. Majumber, Air Force Research Lab., U.S.A 


\section{Invited Panel Discussion Presentation Topics}

"Challenges of Using Deep Learning for Trusted Sensor Fusion"

Dr. Chee-Yee Chong, Independent Consultant

"Deep Learning and Computer Vision: Guidelines and Special Issues"

Professor Lynne Grewe and Garrett Stevenson, California State Univ, East Bay, CA

“ Deep Learning for Object Recognition from High Volume Radio Frequency Data"

Dr. Uttam K. Majumber, Air Force Research Lab.

"Retrospectives on the Application of AI \& Deep Learning in Information Fusion" (addendum to presentations)

Dr. Ivan Kadar, Interlink Systems Sciences, Inc. 


\section{Challenges of Using Deep Learning for Trusted Information Fusion}

Chee-Yee Chong

Independent Researcher

Los Altos, USA

cychong@ieee.org

Presented at Panel on Deep Learning in $\mathrm{Al}$ and Information Fusion

SPIE Signal Processing, Sensor/Information Fusion, and Target Recognition XXVII

Orlando, Florida

April 16, 2018

\section{Outline}

- History of Al for information fusion

- Deep learning benefits and issues

- Framework for trusted information fusion 


\section{Artificial Intelligence (AI) Is Everywhere}

- Al has recent spectacular successes defeating humans

- Deep Blue beat world champion in chess (1997)

- Watson won US Jeopardy quiz show (2011)

- AlphaGo beat world's top player (Ke Jie) in go (2017)

- Al is in everyday applications

- Smart phones - speech recognition, machine translation

- Homes - smart thermostat, robotic vacuum

- Cars - driver assistance

- In particular, Al/deep learning is used in sensor, data and information fusion, e.g.,

- BBC news, 4/13/2018: Chinese police have used facial recognition technology to locate and arrest a man who was among a crowd of 60,000 concert goers.

\section{Al Poses Challenges to Information Fusion}

- Al is viewed as the solution for all fusion problems

- Low-level processing and object recognition

- Video surveillance

- Activity detection and behavioral analysis

- Network and patterns of life analysis

- Al/deep learning is more visible due to

- Beating humans in many applications

- Widely available hardware, software, and training data for development

- Successful use in many systems

- Results

- Sponsors turn to Al to solve information fusion problems

- Al attracts more students, researchers, and funding

- Virtuous cycle (funding -> research -> success -> more funding) 


\section{Information Fusion is a Natural Application for Al}

- Humans solve information fusion problems all the time

- Low level perception - environment, objects

- High level understanding - situation, prediction

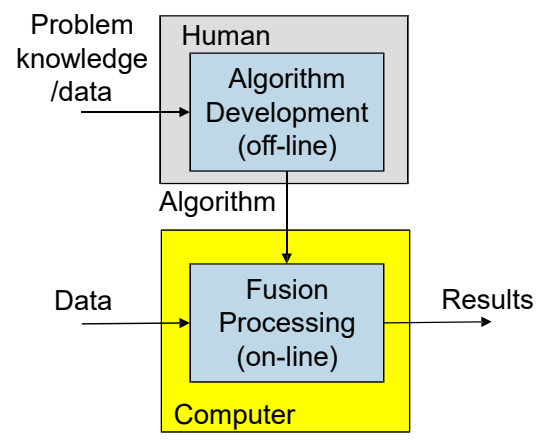

Traditional approach relies on humans to develop algorithm

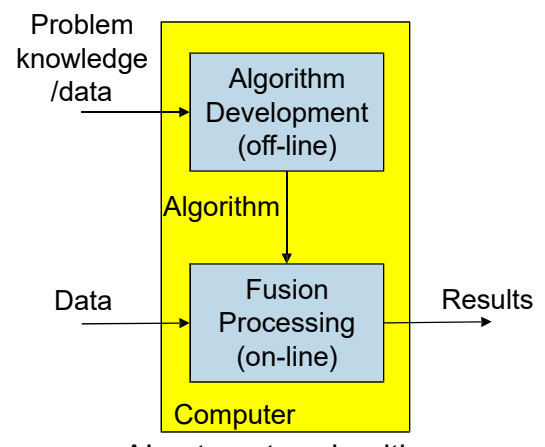

Al automates algorithm development

\section{Al Approaches for Information Fusion Are Evolving}

- Expert systems (mimic human experts)

- Medical diagnosis

- Signal understanding

- Probabilistic reasoning (with models)

- Object recognition

- Situation understanding

- Neural networks / deep learning

- Feature extraction

- Speech understanding

- Object recognition

- Video tracking

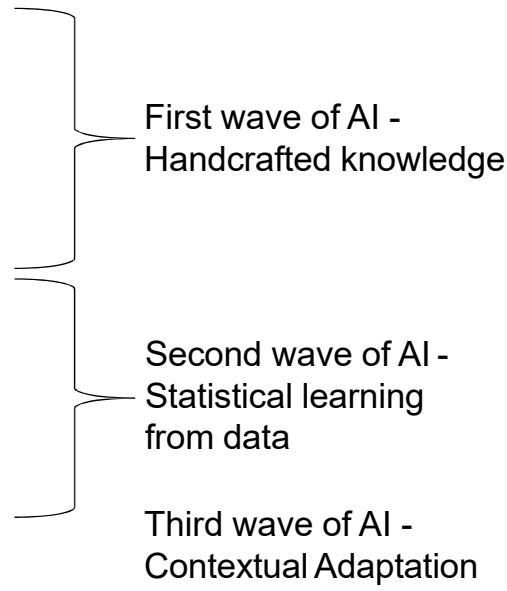

J. Launchbury, "A DARPA perspective on artificial intelligence," 2017 http://www.darpa.mil/attachments/AIFull.pdf 


\section{Expert Systems for Fusion - MYCIN ( 1975)}

- Medical diagnosis

- Inputs: test results

- Outputs: infectious disease

- Rule-based system

- Knowledge base of few hundred IF_THEN rules: IF symptom A THEN disease $B$

- Inference engine by backward chaining

- Certainty factors to represent uncertainty

- Heuristic combination of evidence

- Performs better than many doctors

- Stimulated research on uncertainty reasoning

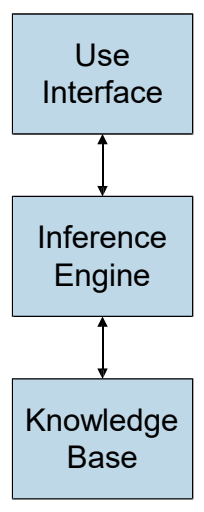

B. G. Buchanan and E. H. Shortlife, Rule-Based Expert Systems: The MYCIN

Experiments of the Stanford Heuristic Programming Project, Addison Wesley, 1984

\section{Expert Systems for Fusion - HASP/SIAP (1970's)}

- Signal understanding system $\quad \substack{\text { Situation } \\ \text { Board }}$

- Inputs: acoustic signals from hydrophones

- Outputs: detection, location and type of vessel

- Rule-based system

- Hierarchy of rules for signal to symbol transformation

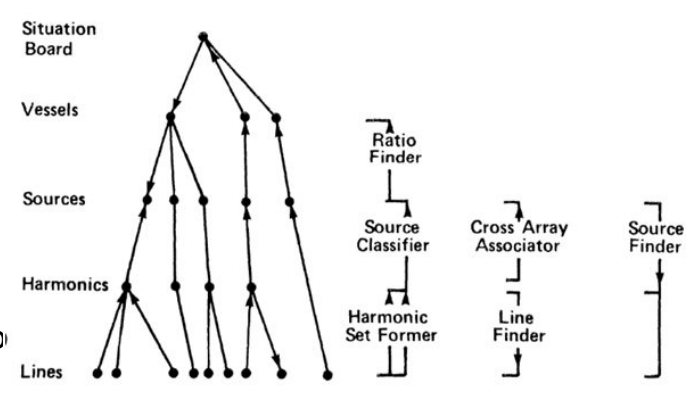

- Inference by knowledge sources responsible for different levels of processing

H. P. Nii, E. A. Feigenbaum, J. J. Anton, "Signal-to-symbol transformation: HASP/SIAP case study," Al Magazine, 1982

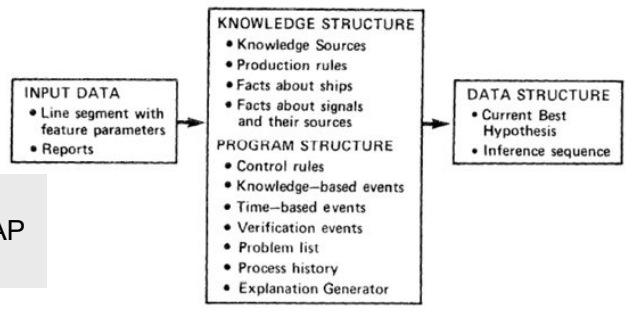




\section{Issues with Expert Systems for Information Fusion}

- Knowledge acquisition

- Finding experts that can articulate their reasoning; experts with good intuition are not suitable

- Extracting knowledge from experts

- Knowledge representation

- Consistency and completeness of rules

- Representation of uncertainty

- Inference engine

- Control of inference

- Reasoning with uncertainty

- Processing speed

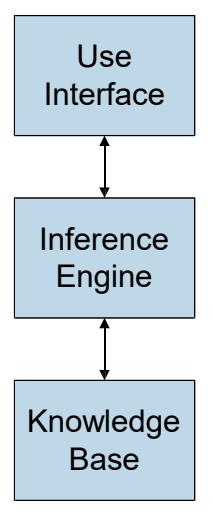

\section{Al in 1980's}

- 1981 - Japan started "Fifth Generation Computer" project to build intelligent computers

- United States responded with "Strategic Computing Initiative" with Al as main objective, including Autonomous Land Vehicle (ALV)

- ALV followed road in 1985 demo but vision system was very sensitive to

- Light and shadow - detect road edge at noon, but not with shadow at dusk

- Environmental change (like mud left along road by another vehicle)

- Booming Al industry (software, hardware) became a bust with Al winter in late 1980's

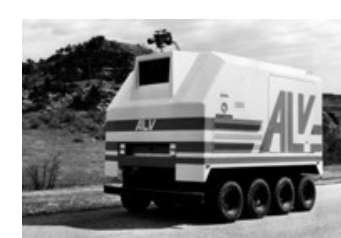

J. E. Franklin, C. L. Carmody, K. Kellter, T. S. Levitt, B. L. Buteau, "Expert system technology for the military: selected samples," IEEE Proceedings, Oct. 1988. 


\section{Neural Networks (1980's)}

- Motivated by physiology and function of neurons in brain

- Long history

- McCulloh, Pitts - 1940's

- Widrow - 1960's

- Hopfield, Rumelhart, Hinton 1980's

-Weights learned from training data

- Excellent for low level recognition task

- Implementation issues

- Black box approach cannot explain results

- Performance sensitive to training data
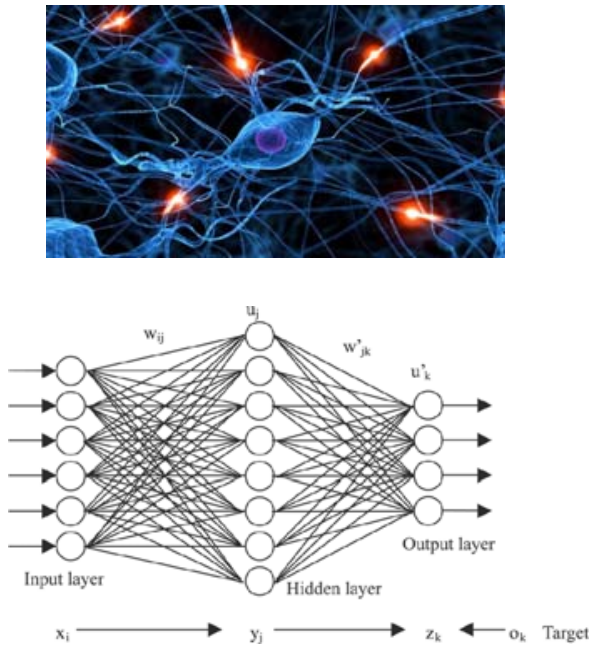

\section{Uncertainty Reasoning in Al}

- Information fusion has deal with uncertainty in evidence (input) and knowledge or data

- Drawback of neural network approach

- Recognized very early by expert system developers

- Uncertainty reasoning approaches

- Rule-based methods

- Probabilistic reasoning

- Evidence theory

- Dempster Shafer

- Dezert-Smarandache Theory (DSmT)

- Fuzzy sets

- Probabilistic reasoning became very popular in 1980's

J. Pearl, Probabilistic Reasoning in Intelligent Systems: Networks of Plausible Inference. Morgan Kaufmann, 1988. 


\section{Probabilistic Reasoning/Graphical Models}

- Probability model expressed graphically as networks

- Nodes are random variables

- Weights on edges represent conditional probabilities

- Inference computes conditional probabilities given evidence

- Node elimination

- Junction tree

- MCMC

- Very natural for researchers with background in estimation theory

- Considered Al because of separation into knowledge and automatic inference
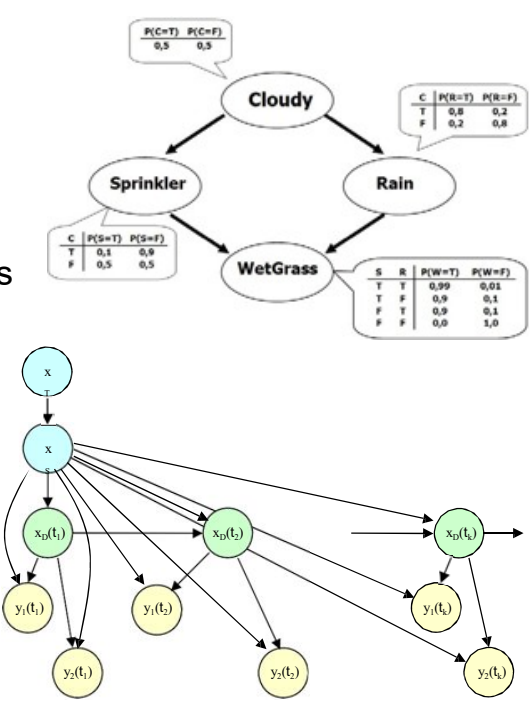

\section{Military Unit Detection from Synthetic Aperture Radar (SAR) Imagery}
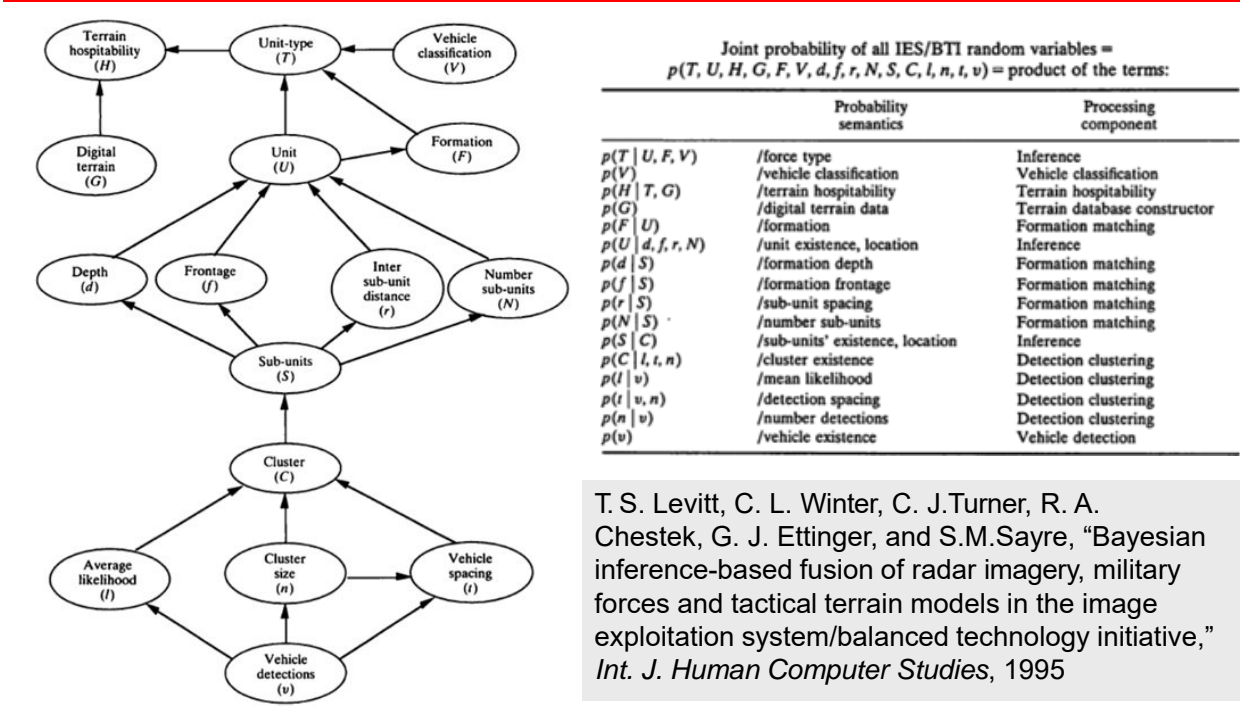

T. S. Levitt, C. L. Winter, C. J.Turner, R. A. Chestek, G. J. Ettinger, and S.M.Sayre, "Bayesian inference-based fusion of radar imagery, military forces and tactical terrain models in the image exploitation system/balanced technology initiative," Int. J. Human Computer Studies, 1995 


\section{Model-Based Object Recognition}

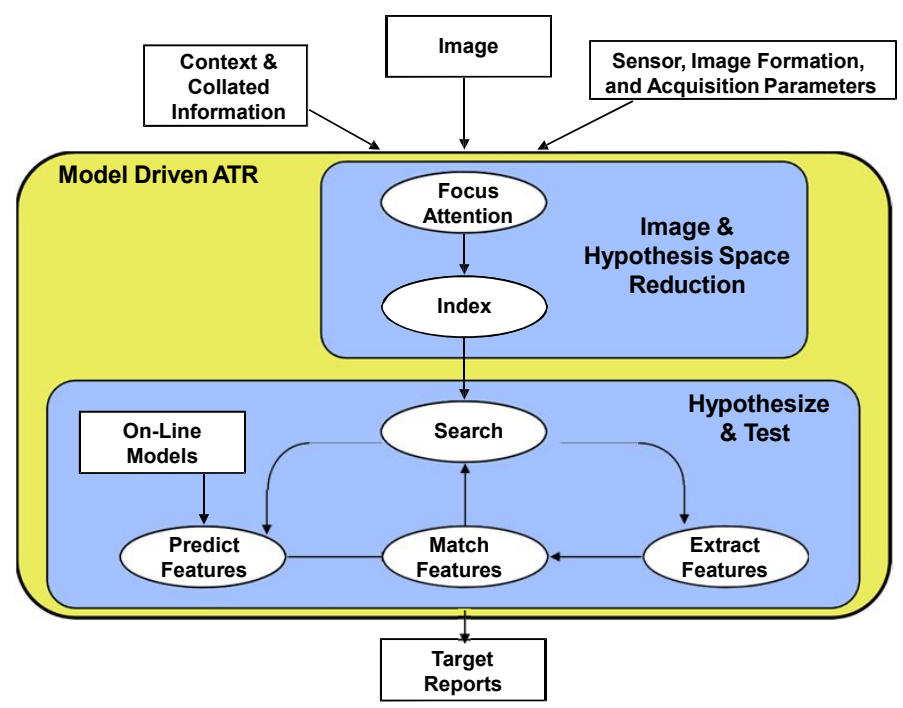

Moving and Stationary Target Acquisition and Recognition (MSTAR)

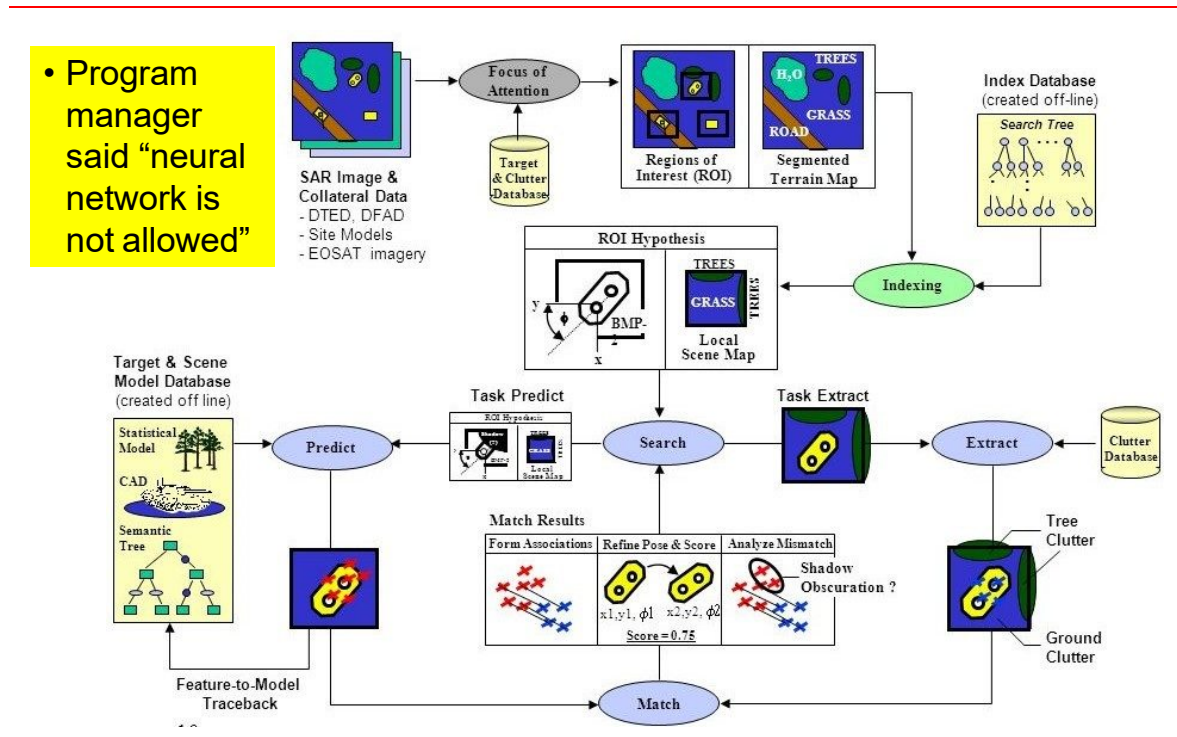




\section{Al for Information Fusion from 1990's to 2010}

- Probabilistic graphical models become main Al approach for fusion

- Rigorous treatment of uncertainty

- Model-based approach is explainable

- Many Inference techniques

- Models can be learned from data

- Can be extended to handle evidence theory, e.g., valuation networks

- Mathematic framework is similar to that of tracking

- Predict features from model

- Match extracted features with prediction (association problem)

- Update

- Meanwhile, neural networks are used for many low level functions where modeling is difficult and training is easy

- Then computers become more powerful and massive amounts of data are available

\section{Shift from Knowledge-Based Al to Learning-Based Al}

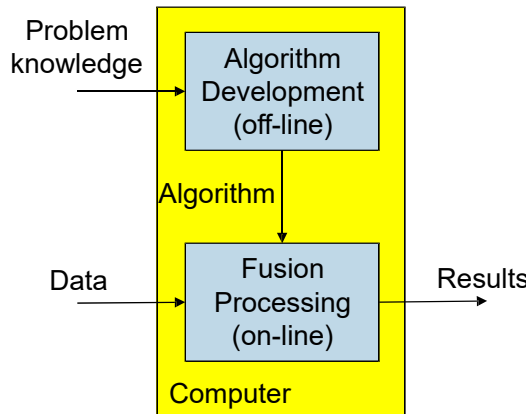

- Explicit problem knowledge

- Manual knowledge acquisition and representation

- Transparent fusion processing

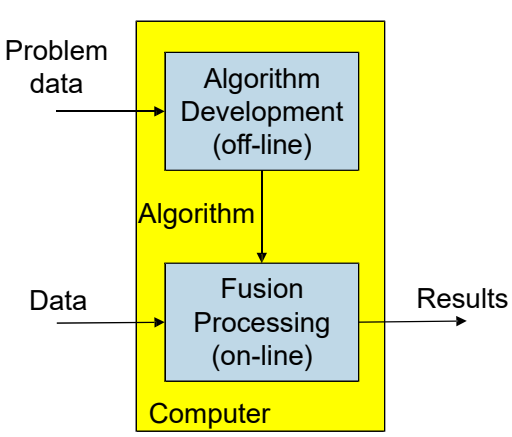

- Problem knowledge captured by data

- Training data acquisition without knowledge representation

- Black box fusion processing 


\section{Deep Learning}

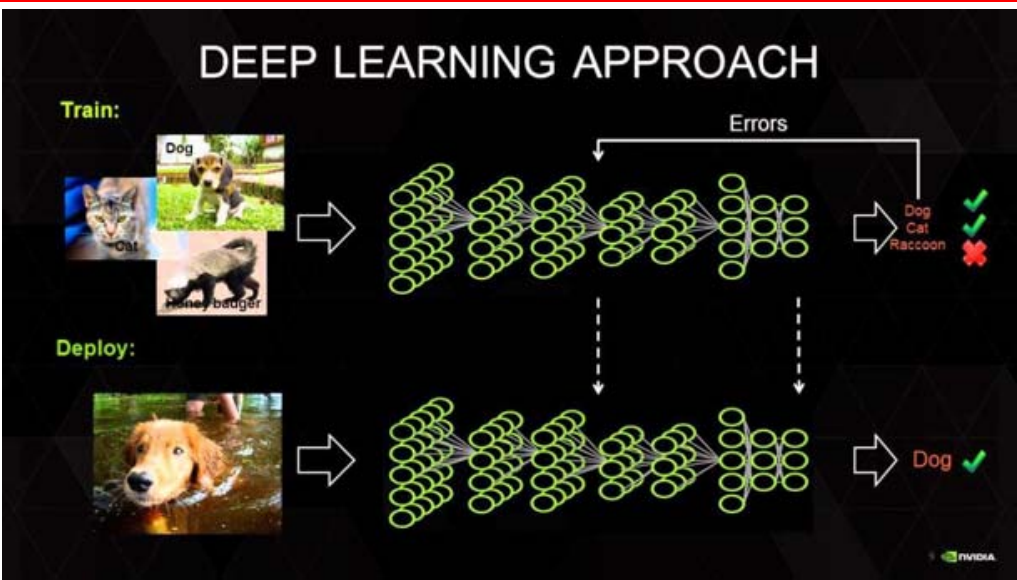

- Deep neural network uses multiple hidden layers between input and output layers to model complex nonlinear relationships

- Input layers can be images or audio signals instead of features

\section{What Makes Deep Learning Possible}

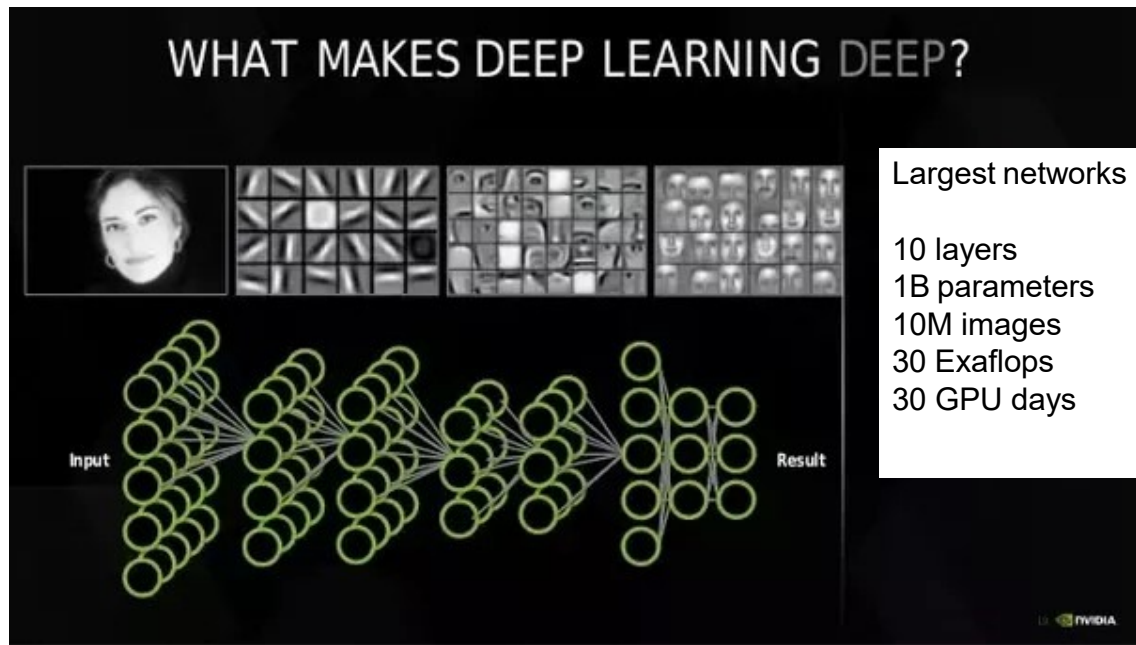

- Deep learning is possible due to advances in computing power and available data 


\section{Deep Learning is Drawing Attention Away From Traditional Information Fusion}

- Almost anyone can apply deep learning for his/her problems

- Open source software, e.g., TensorFlow, Theano

- Public domain data, e.g., ImageNet, Open Images Dataset

- Inexpensive powerful hardware, e.g., Nvidia, Intel, Google

- Deep learning has been successfully used in

- Video surveillance

- Object and threat detection

- Driverless vehicles

- Cyber security (where modeling is very difficult and data is plentiful)

\section{Challenges of Using Deep Learning for Trusted Information Fusion}

- Performance is only as good as data

- Large amounts of data are needed

- Training data for rare events are sparse

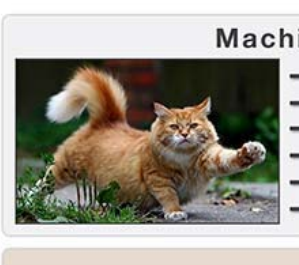

Machine Learning System

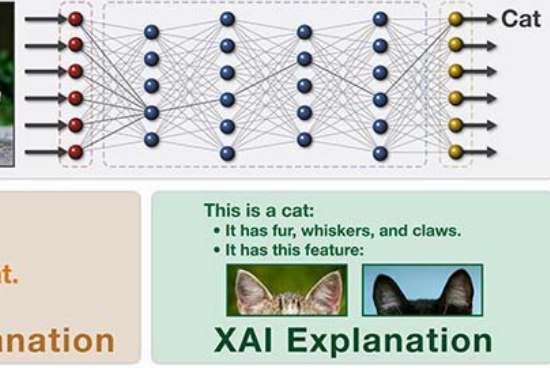

- Results are hard to explain

DARPA Explaniable AI (XAI) Problem

- Black box provides no visibility

- Research on explainable Al is still ongoing

- Thus few machine learning systems are used in critical missions or making life death decisions 


\section{DARPA Explainable Al Program Objective}

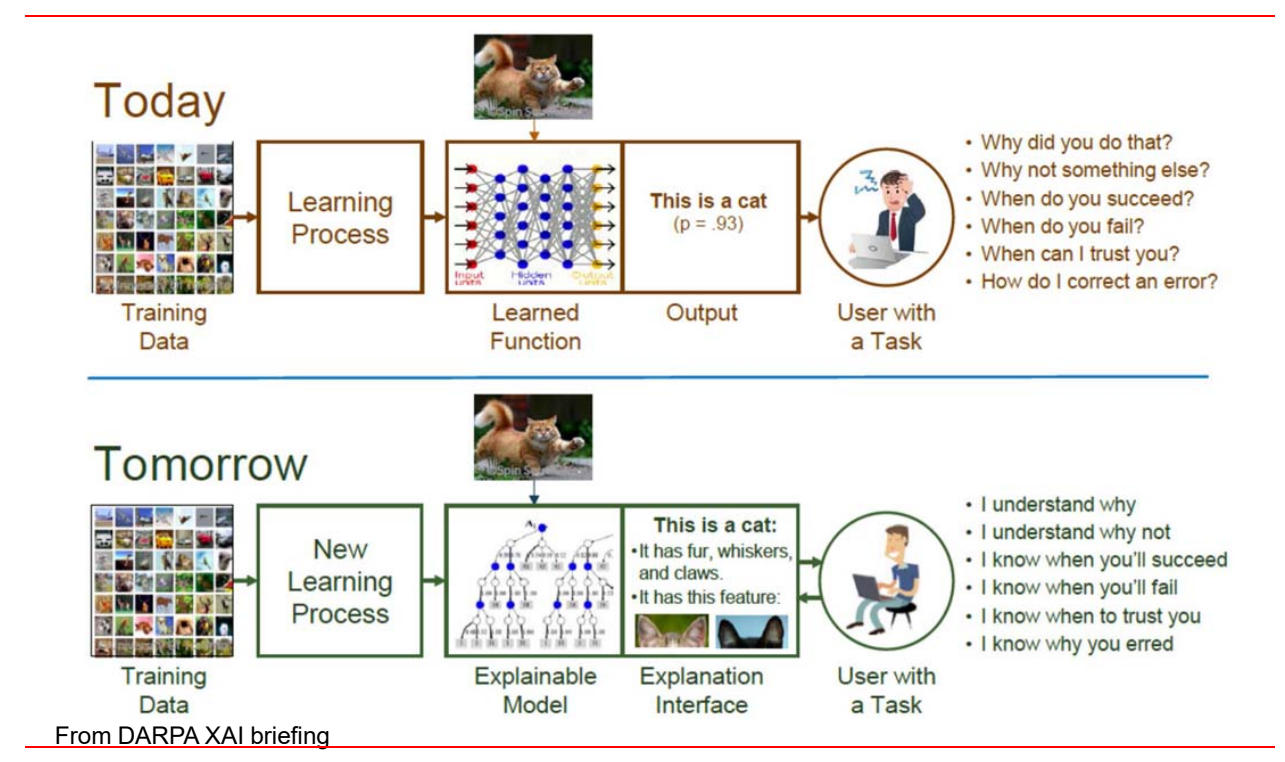

\section{Framework for Developing Trusted Fusion Systems}

- Integrated knowledge and learning approach*

- Inference based on problem knowledge

- Explicit knowledge representation

- Explainable results

- Model parameters from machine learning

- Learning when model knowledge is weak and data are available

- Testable parameter estimation

* ONR solicitation (N00014-18-R-SN05) on integration of domain knowledge and machine learning to address shortcomings of deep learning (large training sets, brittleness, explainability, rare and complex events, etc.) 


\section{Direct Learning of Sensor Input to Object Tracks}

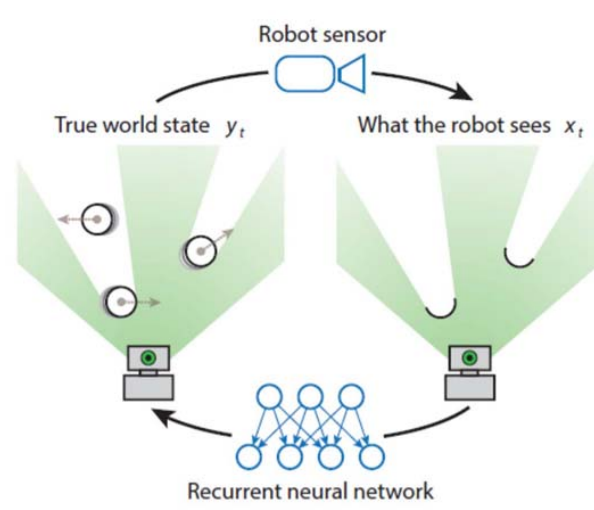

- Related to PHD

- Training and performance issues
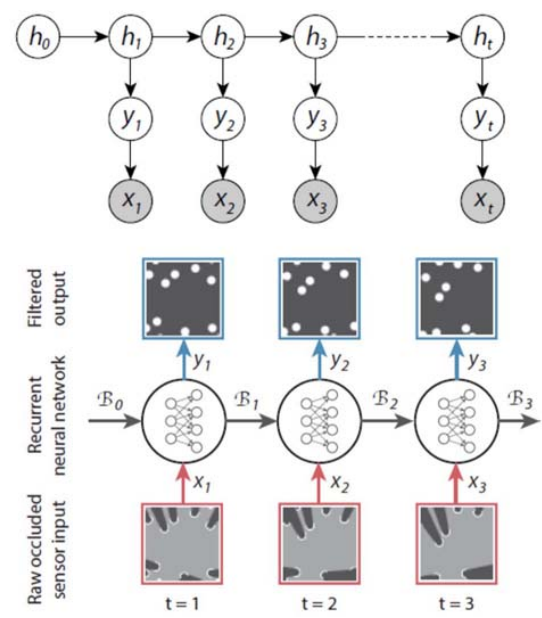

P. Ondruska and I. Posner, "Deep tracking: Seeing beyond seeing using recurrent neural networks," 30th AAAI Conference, 2016

\section{Association Graph for Tracking}

- Association graph provides efficient representation for possible associations

- Nodes: measurements or tracklets

- Edges: possible associations

- Paths: tracks

- Path cover: association hypothesis

- Track likelihood is sum of pairwise association likelihoods under Markov assumptions $P\left(y_{i+1} \mid y_{1}, \ldots ., y_{i}\right)=P\left(y_{i+1} \mid y_{i}\right)$

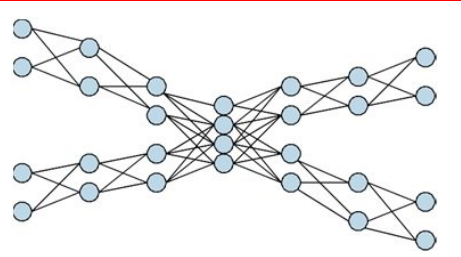

- Best association hypothesis can be computed in polynomial time as

- Bipartite matching

- Minimum cost network flow
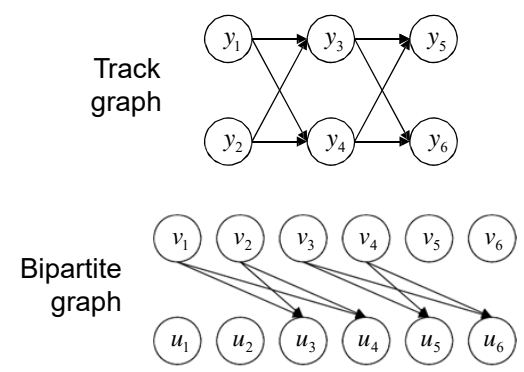

C. Y. Chong, "Graph approaches for data association," Fusion 2012. 


\section{Learning Association Costs in Association Graph}
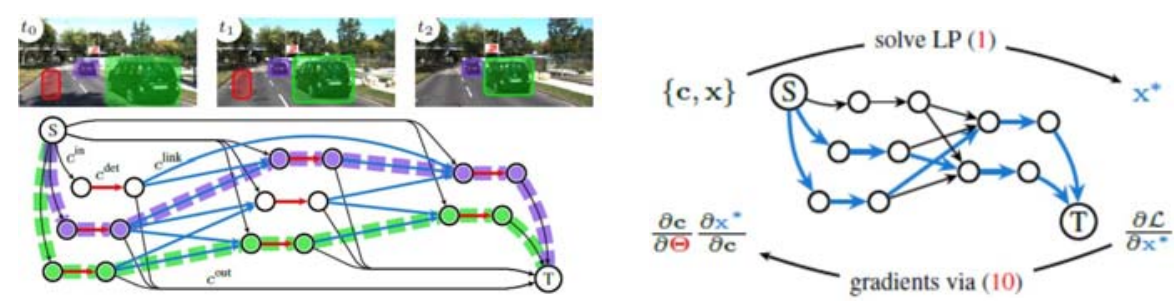

- Pairwise association costs can be computed

- Attributes of detections

- Measurement models

- Alternatively, they can be learned from data using back propagation

S. Schulter, P. Vernaza, W. Choi, and M. Chandraker, "Deep network flow for multi-object tracking," CVPR 2017.

\section{Integrated Knowledge/Learning for Multitarget Tracking}

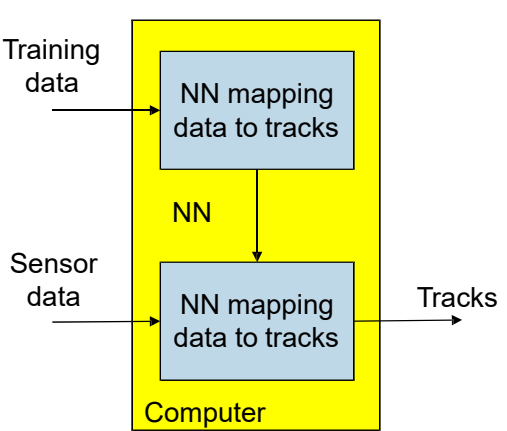

- Direct learning

- Questionable performance

- Hard to explain

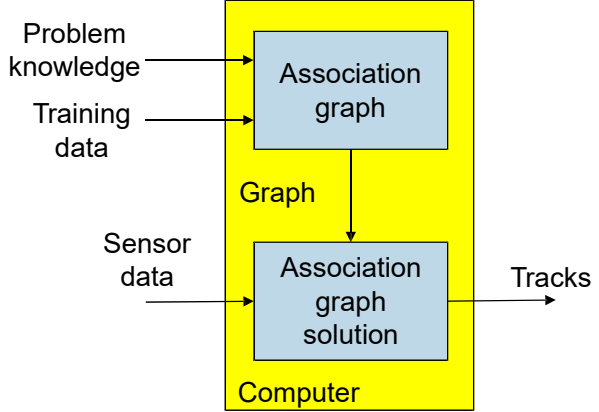

- Integrated knowledge/learning

- Higher performance

- Explainable 


\section{Conclusions}

- Information fusion researchers have love hate relationship with computers - Love to develop algorithms for computers

- Hate when computers become too smart to take over their job

- Users do not care about specific approach as long as it provides solution

- Al and deep learning still cannot solve all fusion problems, especially in trusted or mission critical systems

- Trusted fusion system should exploit

- Knowledge when it is available and can be represented for inference

- Machine learning when knowledge is weak and data are available 
"Deep Learning and Computer Vision: Guidelines and Special Issues "

Lynne Grewe ${ }^{a}$, and Garrett Stevenson ${ }^{a}$

aComputer Science, California State University East Bay,

25800 Carlos Bee Boulevard, Hayward, CA USA, 94542

Deep Learning in Al and Information Fusion Panel

\section{Computer Vision Today \& Deep Learning}

Computer Vision is becoming pervasive in today's society and has a presence in self-driving cars, cities of the future, drones, medicine and more. Central to its use and popularity is the combination of Deep Learning and Computer Vision to tackle the important tasks of object classification and localization.

Recent developments of Computer Vision with Deep Learning will be discussed with guidelines \& special issues 


\section{overview}

- Trends: Vision and Deep Learning architectures

- Running on mobile platforms/embedded devices

- Multi-Modal issues for Vision- a case study

- Temporal Networks - LSTM

- General Adversarial Networks

- Varying data size

- Transfer Learning

- Guidelines

- Resources -datasets, frameworks \& computation

- iLab (my) Deep Learning Research Projects

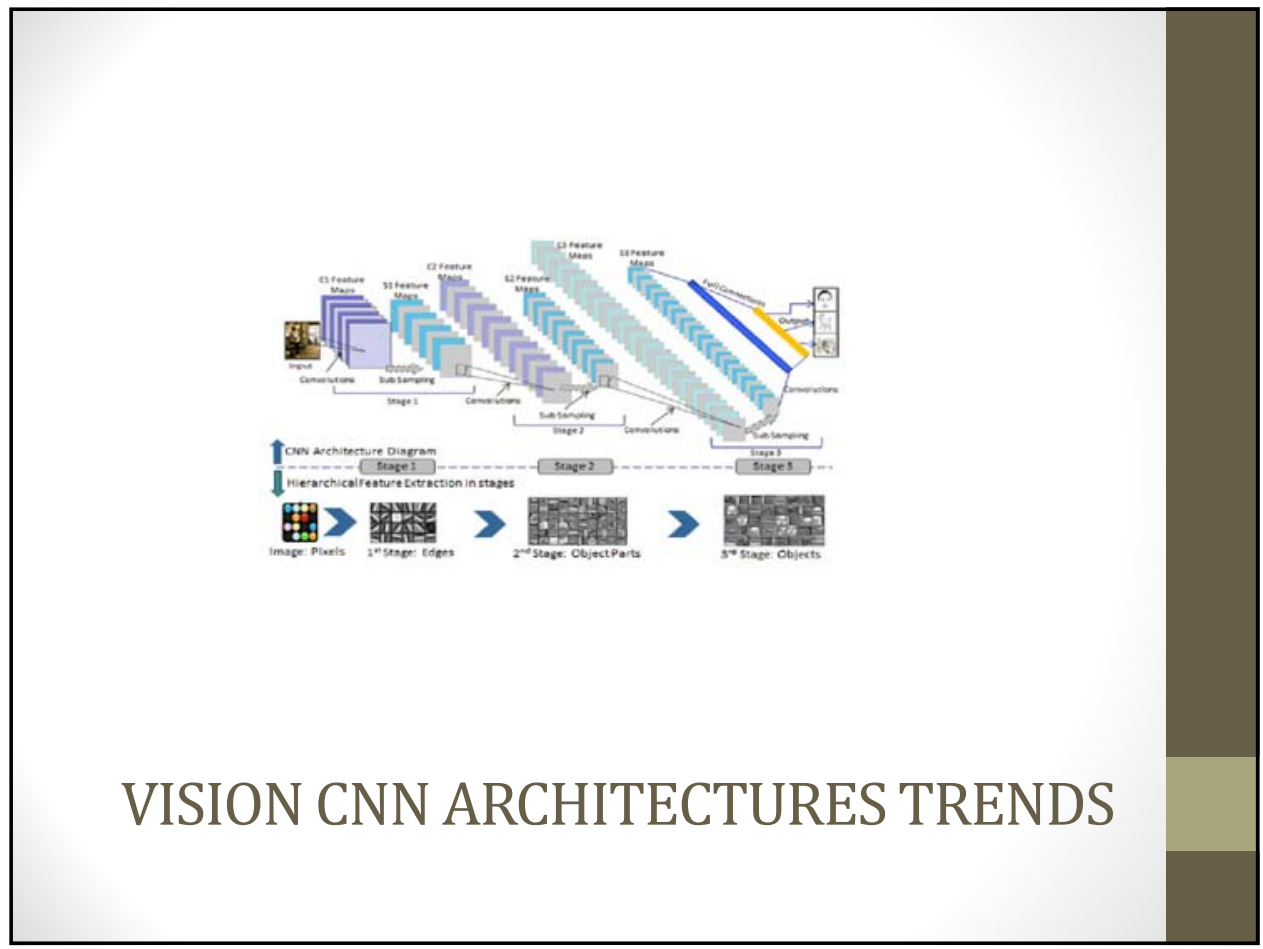




\section{CNN-basics}
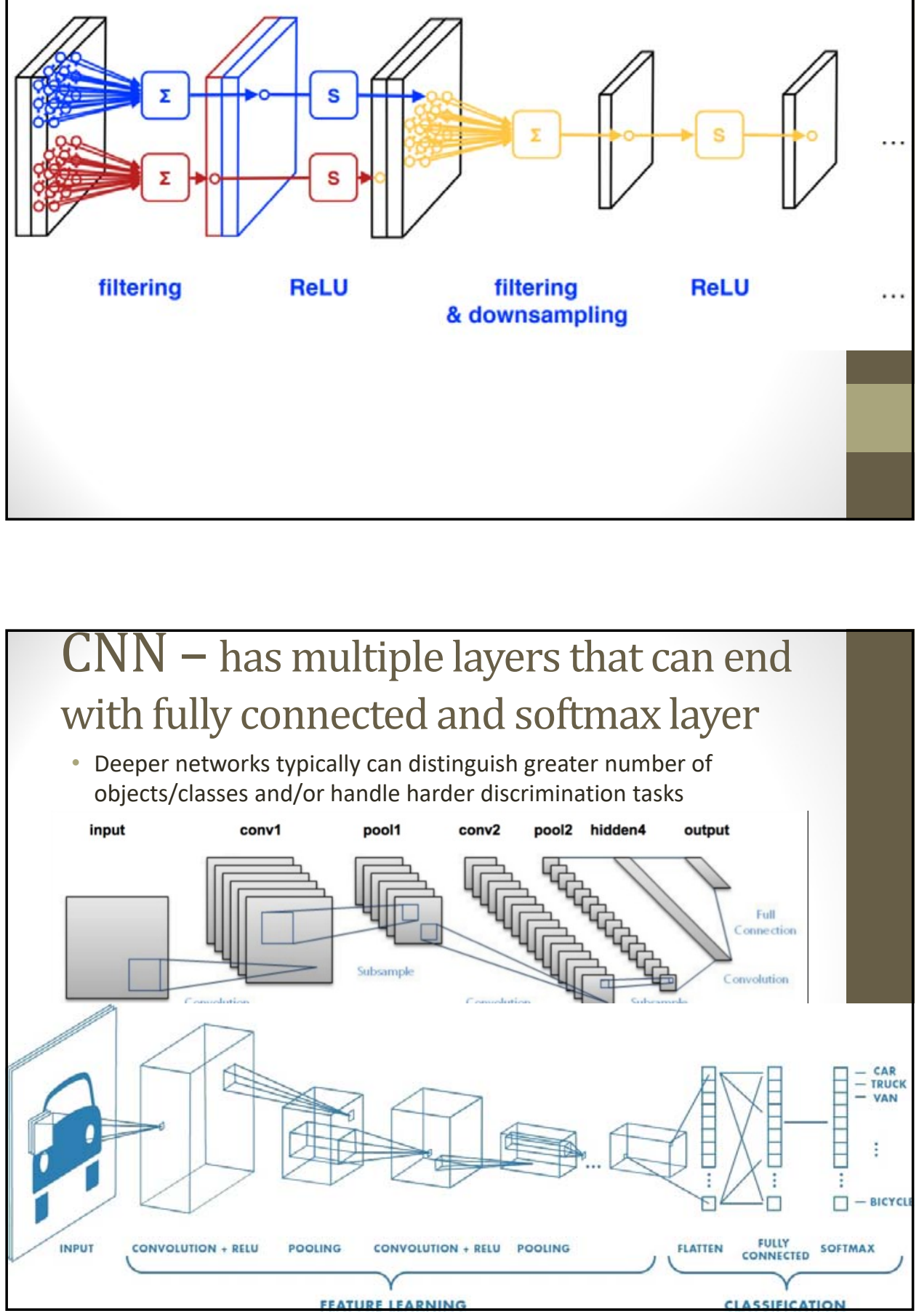

xxxiv 


\section{Shallow CNN for simpler problem of Digit recognition}

- 4 convolutional layers +1 fully connected layer

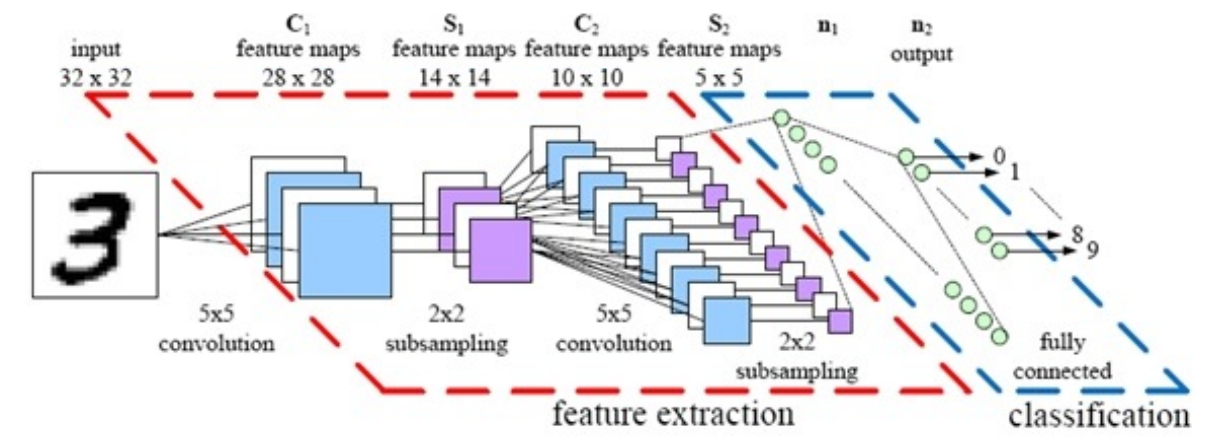

\section{Going Deeper ResNet 152 - has 152 layers !!!!!!!!}

AlexNet

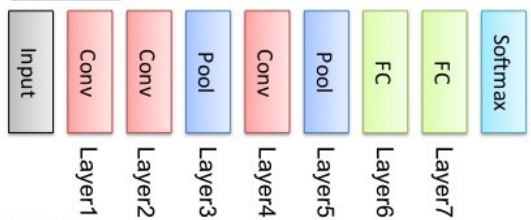

\begin{tabular}{|c|c|}
\hline Input & : Image input \\
\hline Conv & : Convolutional layer \\
\hline Pool & : Max-pooling layer \\
\hline $\mathrm{FC}$ & : Fully-connected laye \\
\hline Softma & : Softmax layer \\
\hline
\end{tabular}

VGGNet 


\title{
Problem -Deeper Network generally slower
}

\author{
- Resnet -152 is 5 times slower than Alexnet
}

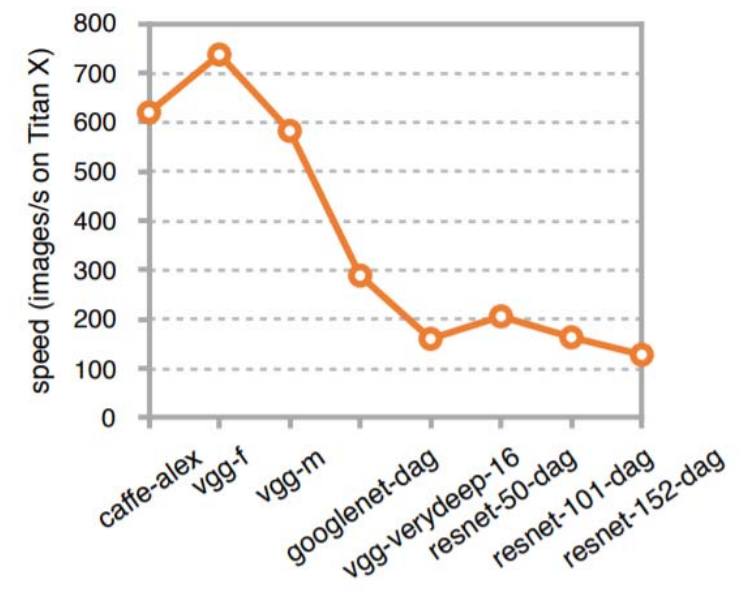

\section{No Fully Connected Layers}

There is ALL kinds of research going on in architectures for Deep Learning Networks sometimes you may have different architectures suited for different problems

- Fully Convolutional Networks for Semantic Segmentation (no fully connected layers)

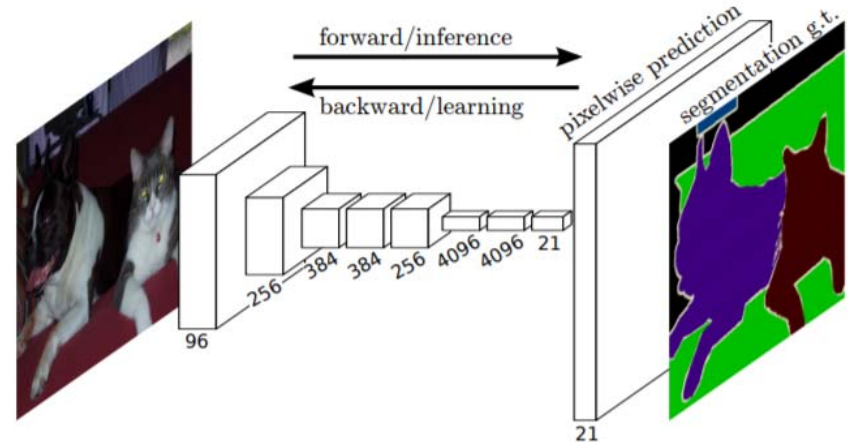

Figure 1. Fully convolutional networks can efficiently learn to make dense predictions for per-pixel tasks like semantic segmen- 


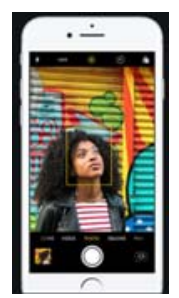

When want to run on device

\section{LOW COMPUTATION - MOBILE AND EMBEDDED DEVICES}

\section{MobileNet - uses Depthwise Separable} parameters to reduce number of parameters

- Convolution $=$ depthwise + pointwise

- perform a spatial convolution while keeping the channels separate and then follow with a depthwise convolution.

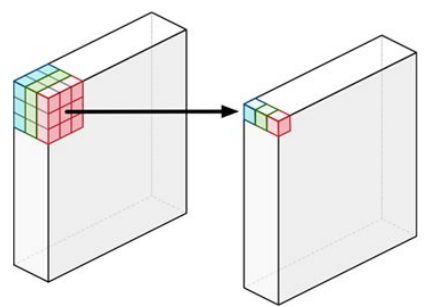

Depthwise

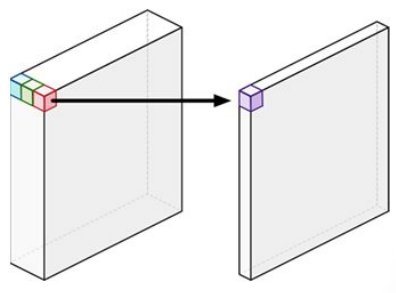

pointwise 


\section{MobileNet-less parameters}

- For a depthwise separable convolution on the same example, we traverse the 16 channels with $13 \times 3$ kernel each, giving us 16 feature maps (called DepthWise Convolution). Now, before merging anything, we traverse these 16 feature maps with 32 $1 \times 1$ convolutions each (called Pointwise convolution) and only then start to them add together. (this $1 \times 1$ is called a depthwise multiplier of 1 )

Comparison of number of parameters --> SEPARATED IS LESS!!!

- This results in $656(16 \times 3 \times 3+16 \times 32 \times 1 \times 1)$ parameters opposed to the $4608(16 \times 32 \times 3 \times 3)$ parameters from above.

SO its faster

\section{MobileNet}

- You can choose number of layers ...example here with 9

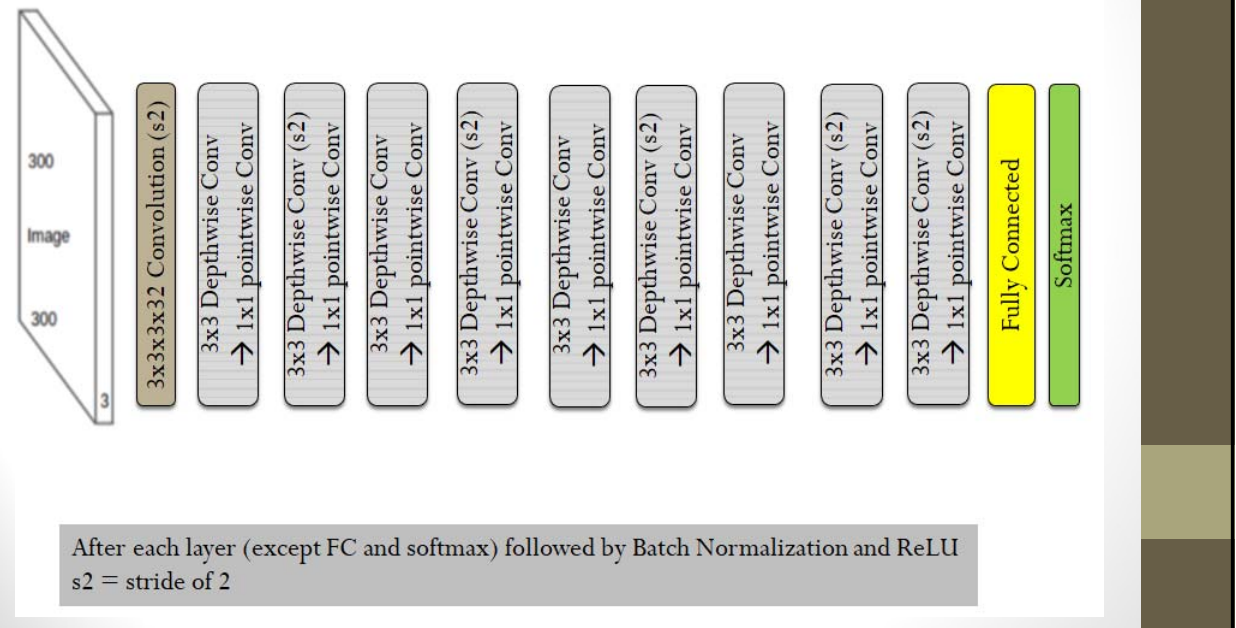

xxxviii 


\section{TensorFlow Lite}

- Framework = Target mobile and embedded devices

- enables on-device machine learning inference with low latency and a small binary size.

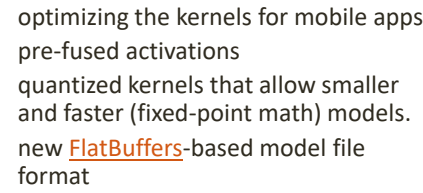

optimizing the kernels for mobile apps pre-fused activations

quantized kernels that allow smaller and faster (fixed-point math) models.

new FlatBuffers-based model file format

Near future will add SSD -localization And hopefully soon with NN API hardware acceleration

- smaller than 300KB when all supported operators are linked and less than 200KB when using only the operators needed for supporting InceptionV3 and Mobilenet.

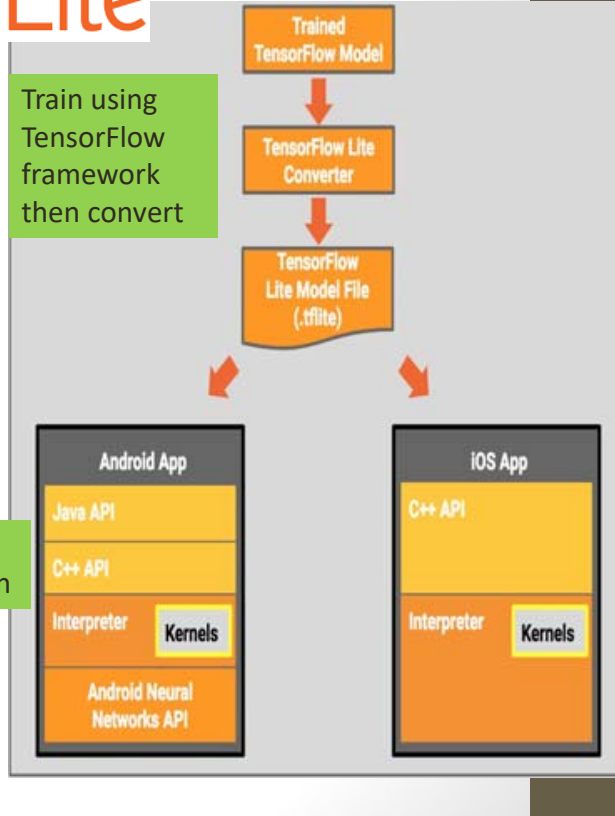

- Java and C++ API support

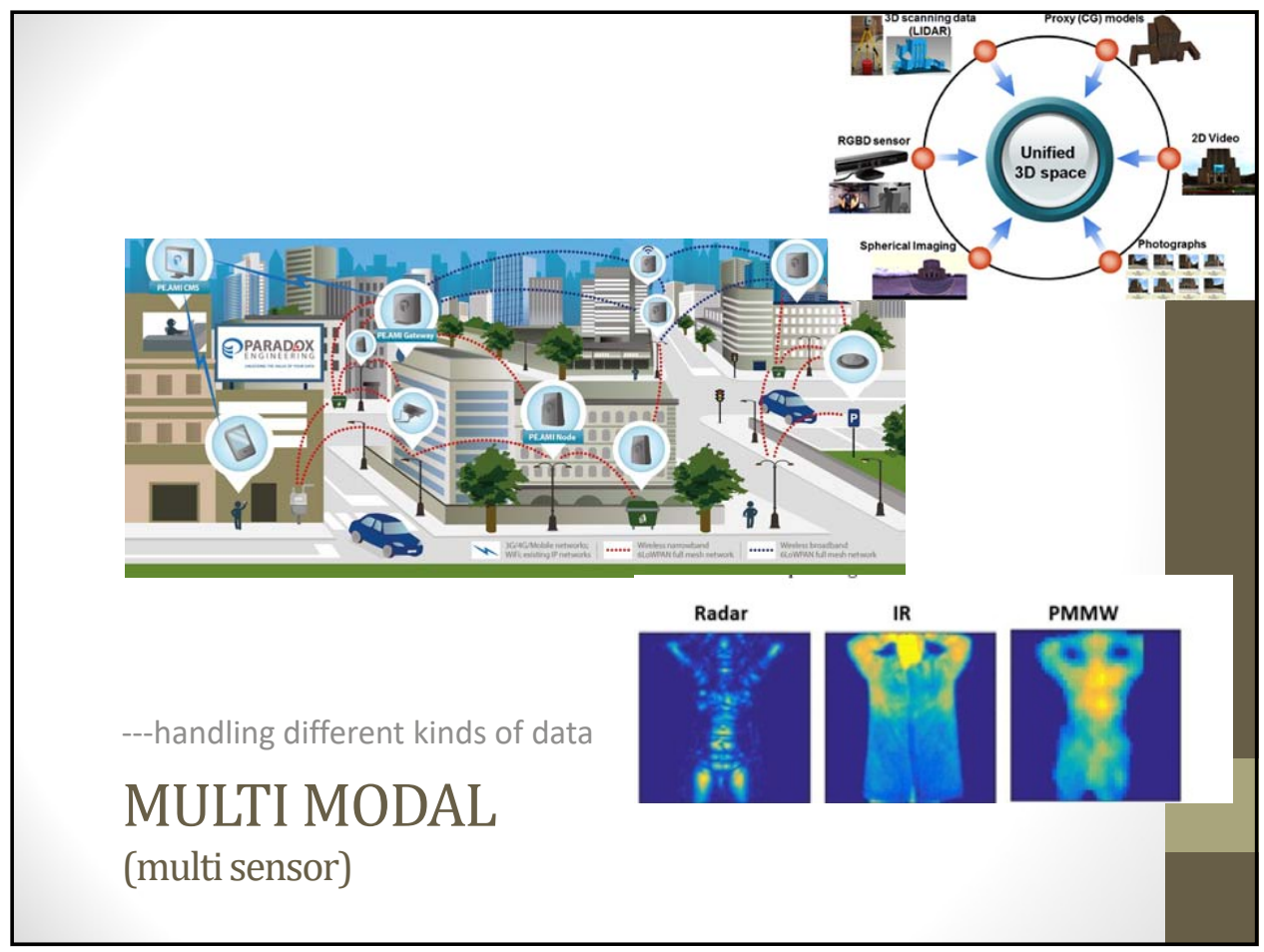




\section{Multi Modal (multi sensor)}

- Have multiple different data from different sensors.

- CASE 1: Data Aligns spatially

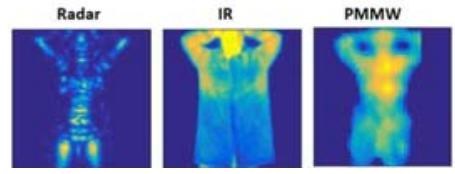

- CASE 2: Data does not align spatially
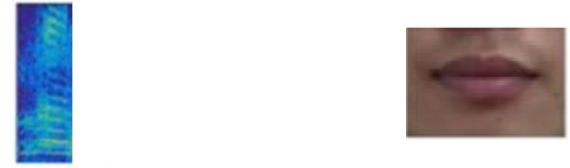

speech frames Cropped mouth images

\section{CASE 1: data "aligns" spatially}

\section{Questions:}

- Do you process both in the CNN

- Do you have multiple CNNs? How do you merge results?

- How will this effect Region Proposal (if doing localization)?

- How will this effect speed?

\section{Options:}
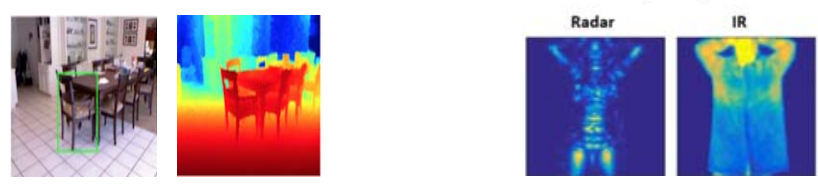

PMMW

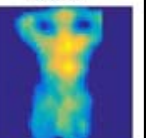

OPTION 1: Driven by one modality (e.g. 2D rgb processed first)

OPTION 2: Process in parallel with separate networks OPTION 3: Process together with 1 network 


\section{OPTION 1: single data driven $\rightarrow$ use other data later in process}

- From B. Chen, R. Sahdev, J. Tsotsos, "Integrating stereo vision with a CNN tracker for a person-following Robot", International Conference on Computer Vision Systems, 2017

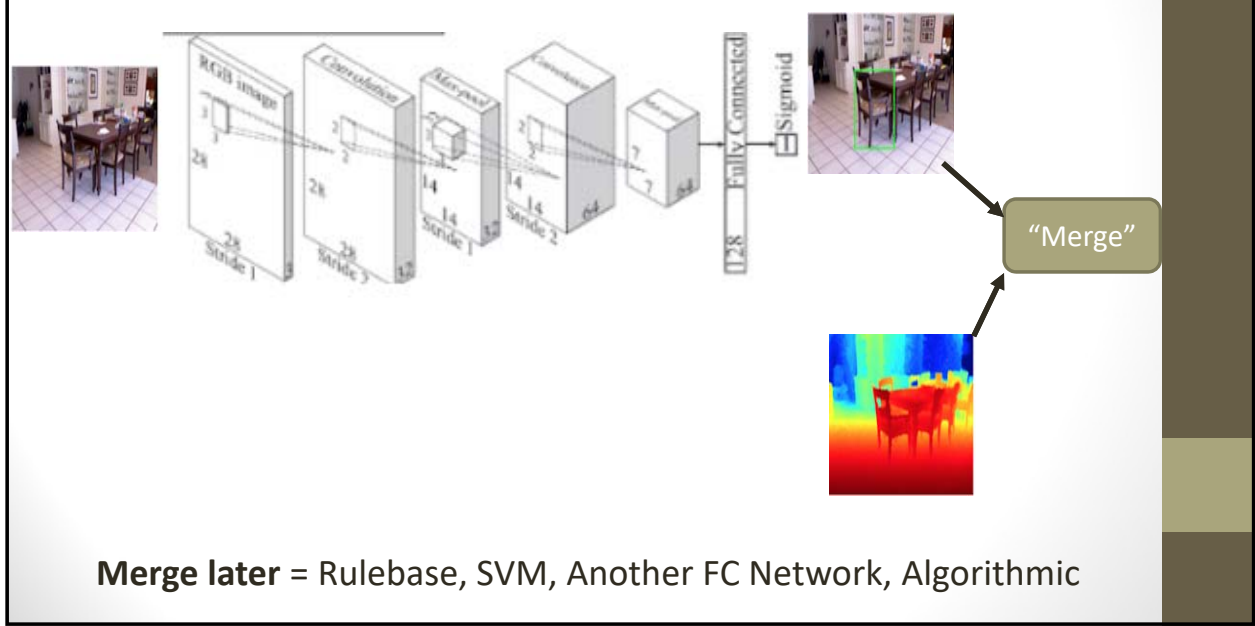

\section{OPTION 2: separate CNN run in parallel} for each sensor data

Must combine results of separate CNNs:

- Fully connected layer

- SVM

- Rule-based / algorithmic combination
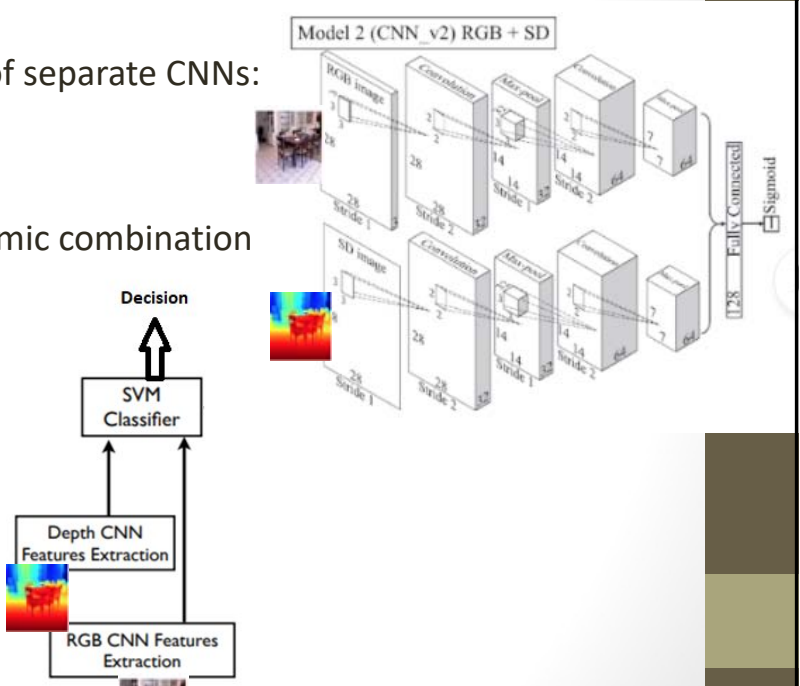

12en 

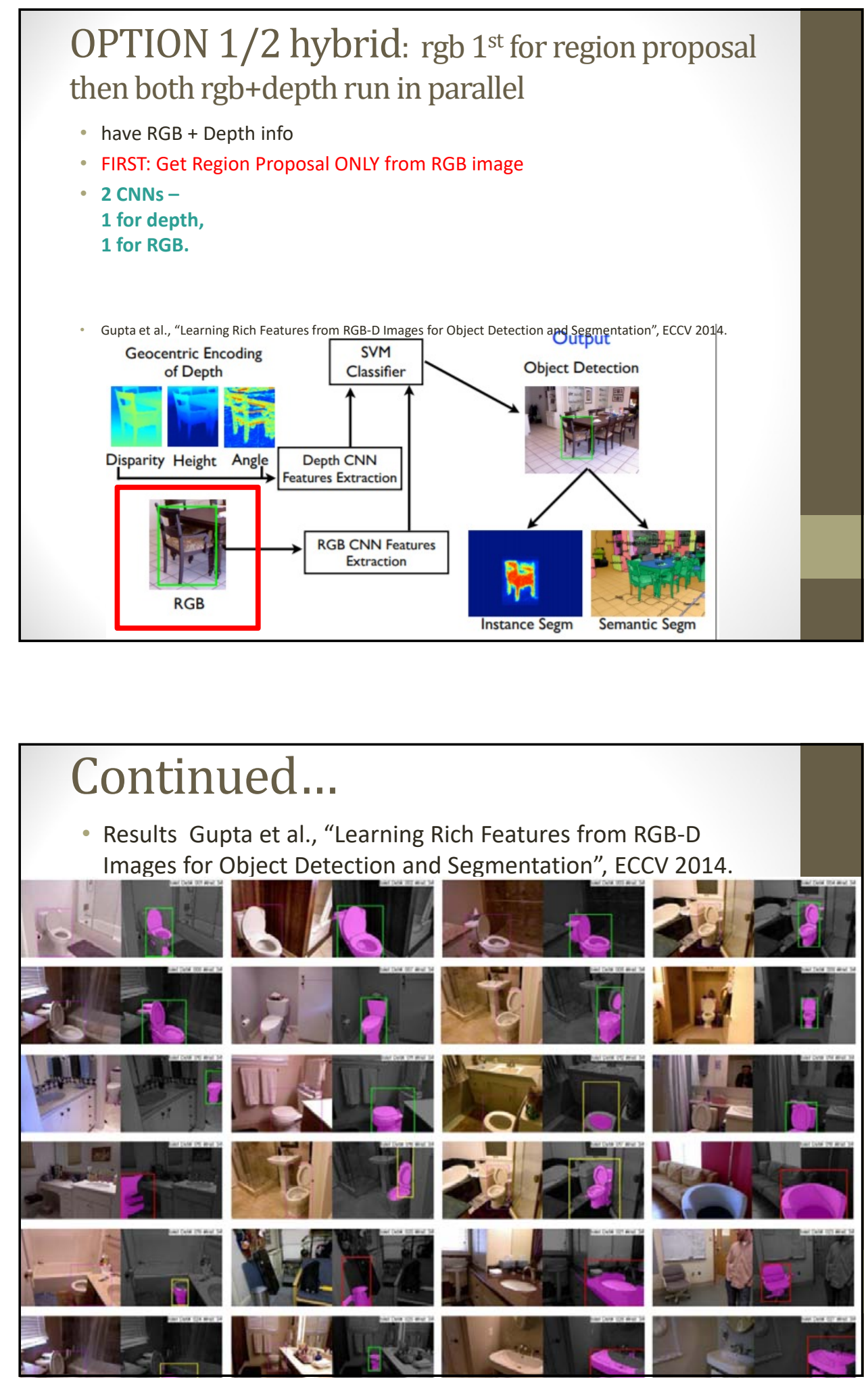


\section{Option 3: combine data into 1}

CNN

From B. Chen, R. Sahdev, J. Tsotsos, "Integrating stereo vision with a CNN tracker for a person-following Robot", International Conference on Computer Vision Systems, 2017

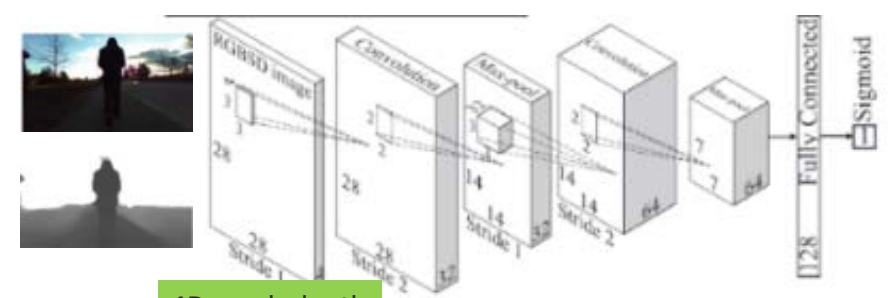

$4 \mathrm{D}=\mathrm{r}, \mathrm{g}, \mathrm{b}, \mathrm{depth}$

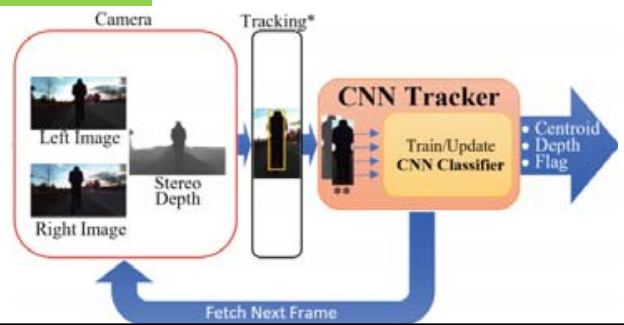

\section{Option 3: another example}

- Here is a NON visual (but, temporal data) sensor example

From L. Jing, T. Wang, M. Zhao, P. Wang, "An Adaptive Multi-Sensor Data fusion Method Based on Deep Convolutional Neural Networks for Fault Diagnosis of Planetary Gearbox", Sensors 2017

Multi-sensor $\rightarrow$ Data preprocessing $\rightarrow$ Data level fusion $\rightarrow \begin{gathered}\text { Deep convolutional neural networks } \\ \text { based feature learning and data fusion }\end{gathered} \rightarrow$ Diagnosis result

Accelerometer Divide signals into segments Combine four segments
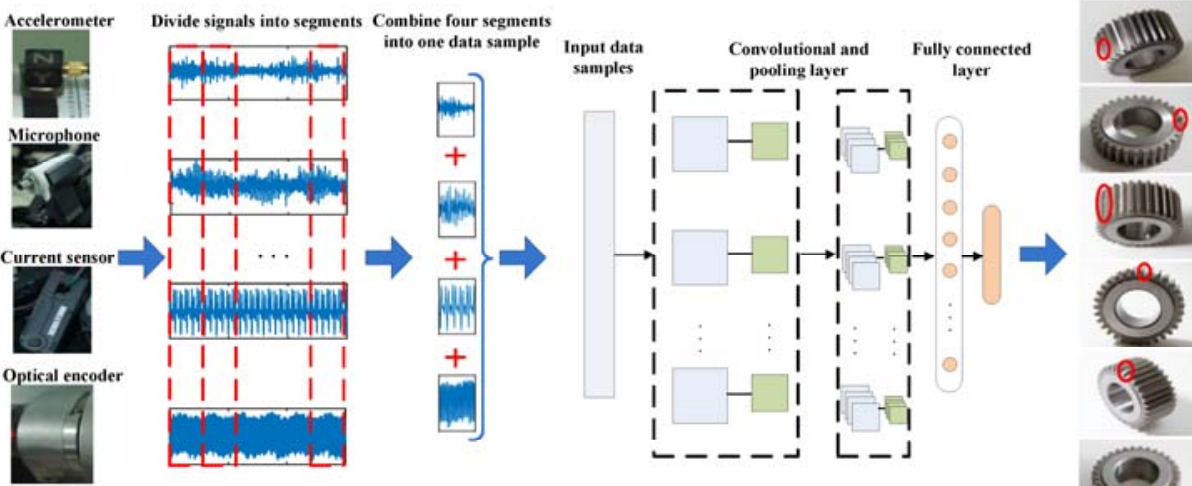


\section{WHICH option is best?}

From B. Chen, R. Sahdev, J. Tsotsos, "Integrating stereo vision with a CNN tracker for a personfollowing Robot", International Conference on Computer Vision Systems, 2017

- Compared options 1, 2, 3 -

for Person Detection \& Tracking with RGB + Depth

\#1= OPTION 3: 1 CNN for all data \#2= OPTION 2: 2 CNN in parallel \#3= OPTION 1: use only 1 sensor
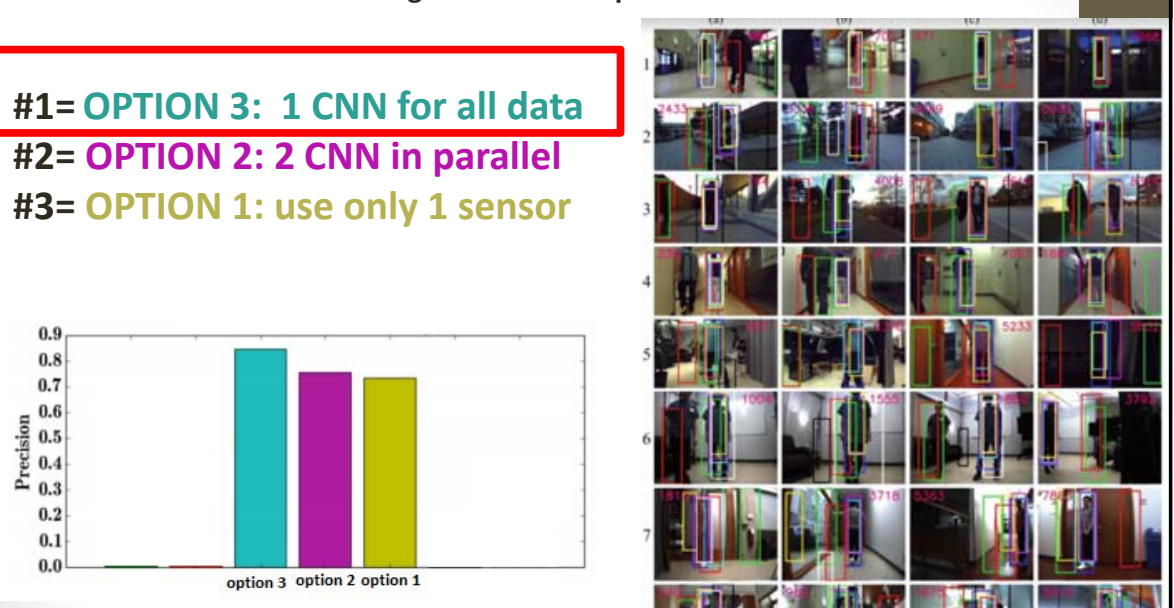

CASE 2: data does not "align" spatially

- Example speech (not spatial, temporal only) and vision

- Process in parallel with separate CNNs

$\rightarrow$ this works because there is temporal alignment

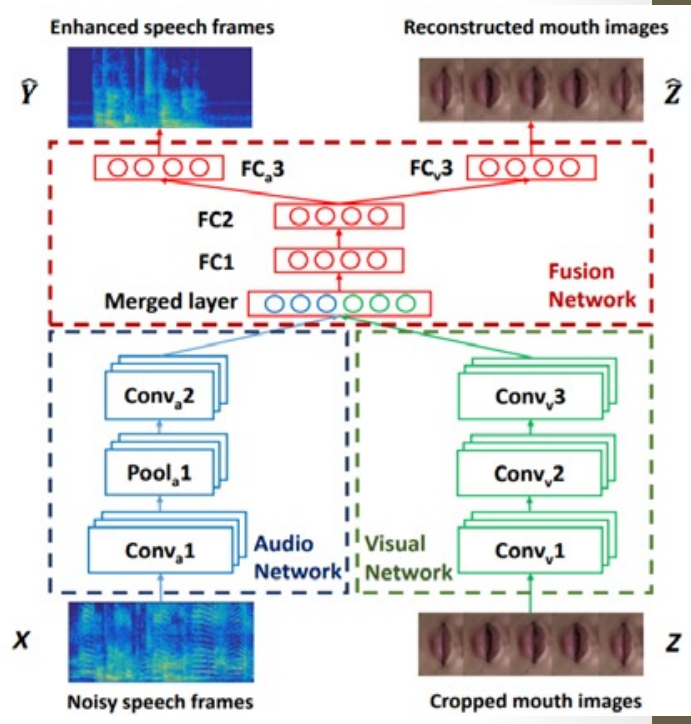




\section{4 - Two steam networks}

From K. Simonyan and A. Zisserman, "Two-Stream Convolutional Networks for Action Recognition in Videos", Neural Information Processing Systems Conference, 2014

- "Separate" Visual and temporal

- 2 CNNs: 1) for single RGB image 2) for optical flow between current and previous frame(s).

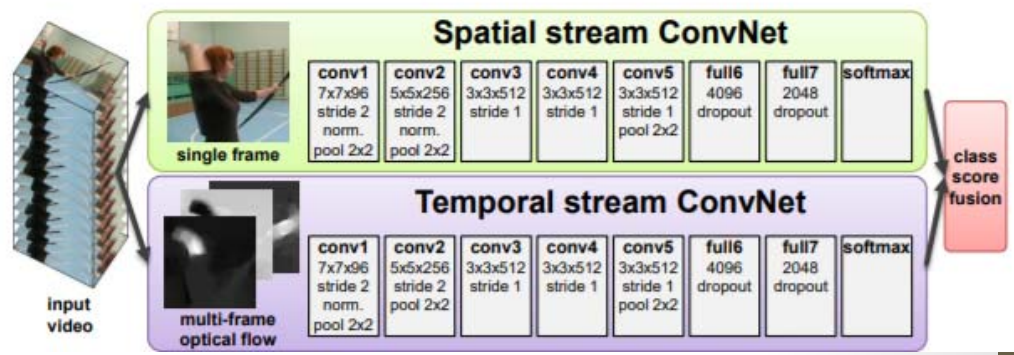




\section{$\mathrm{CNN} \rightarrow \mathrm{RNN}$ (Recurrent Neural Networks)}

- Used in Speech other naturally temporal data (no spatial components)

RNNs in comparison to a traditional CNN do not capture rich spatial information as well.

- One Idea = Combine RNN and CNN in Sequence

Example from

"Show and Tell Me" system

From O. Vinyals, A. Toshev, S. Bengio, D. Erhan,

"Show and Tell: A Neural Image Caption Generator", CVPR, 2015

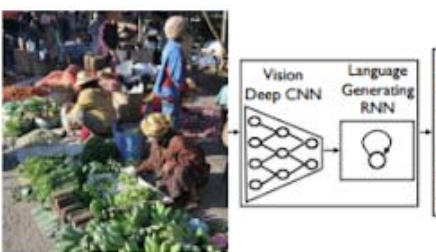

A group of people shopping at an outdoor market.

vegetables at the fruit stand.

Image Captioning

CNN for Vision process $\rightarrow$ RNN for language generation

\section{Problems with RNN}

- "Long Term" Issue = Difficult to backpropogate an error over a long-range temporal span becomes difficult.

- "Short-term" Issue = Basic RNN does not allow network to "forget" previous hidden states. 

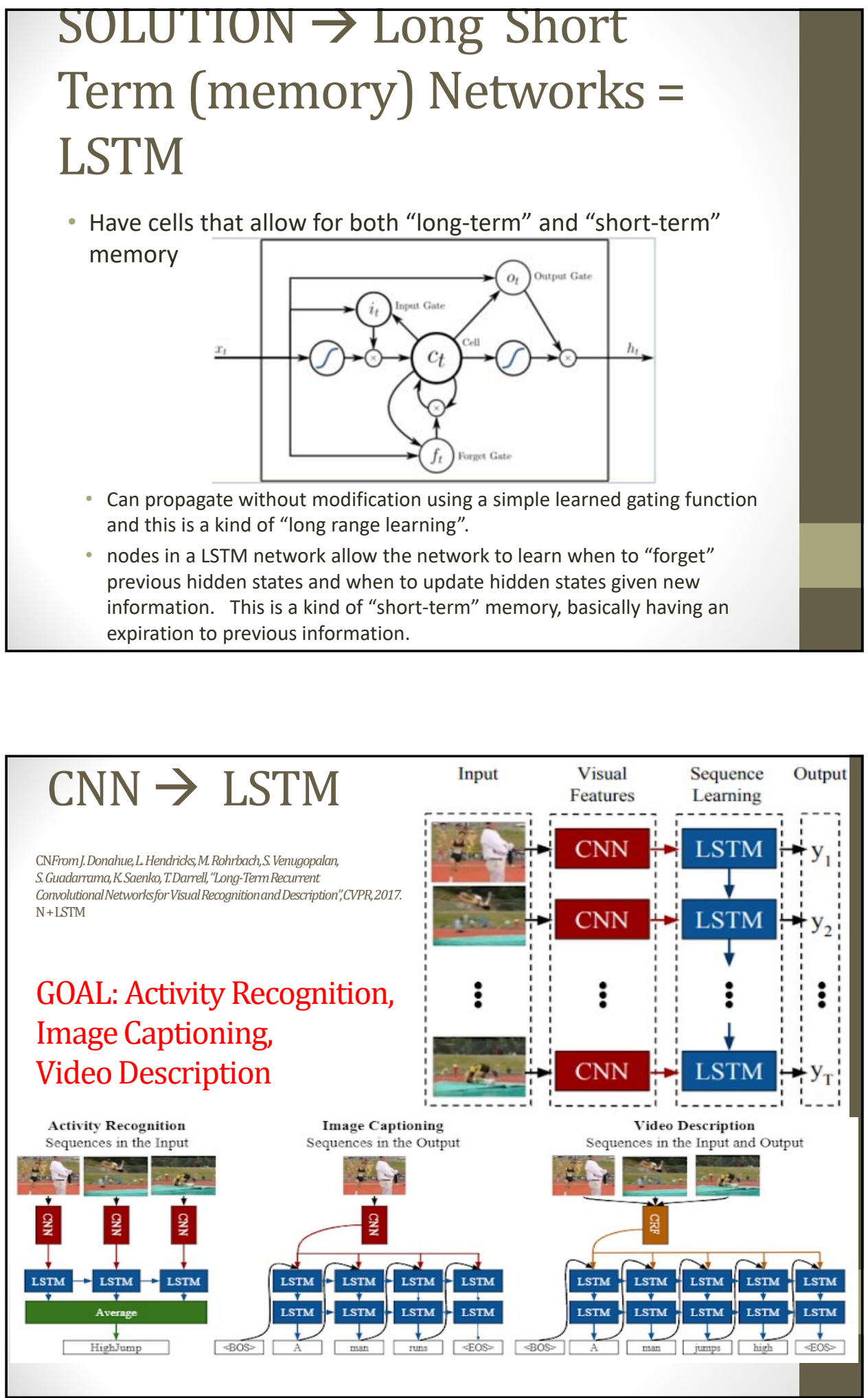

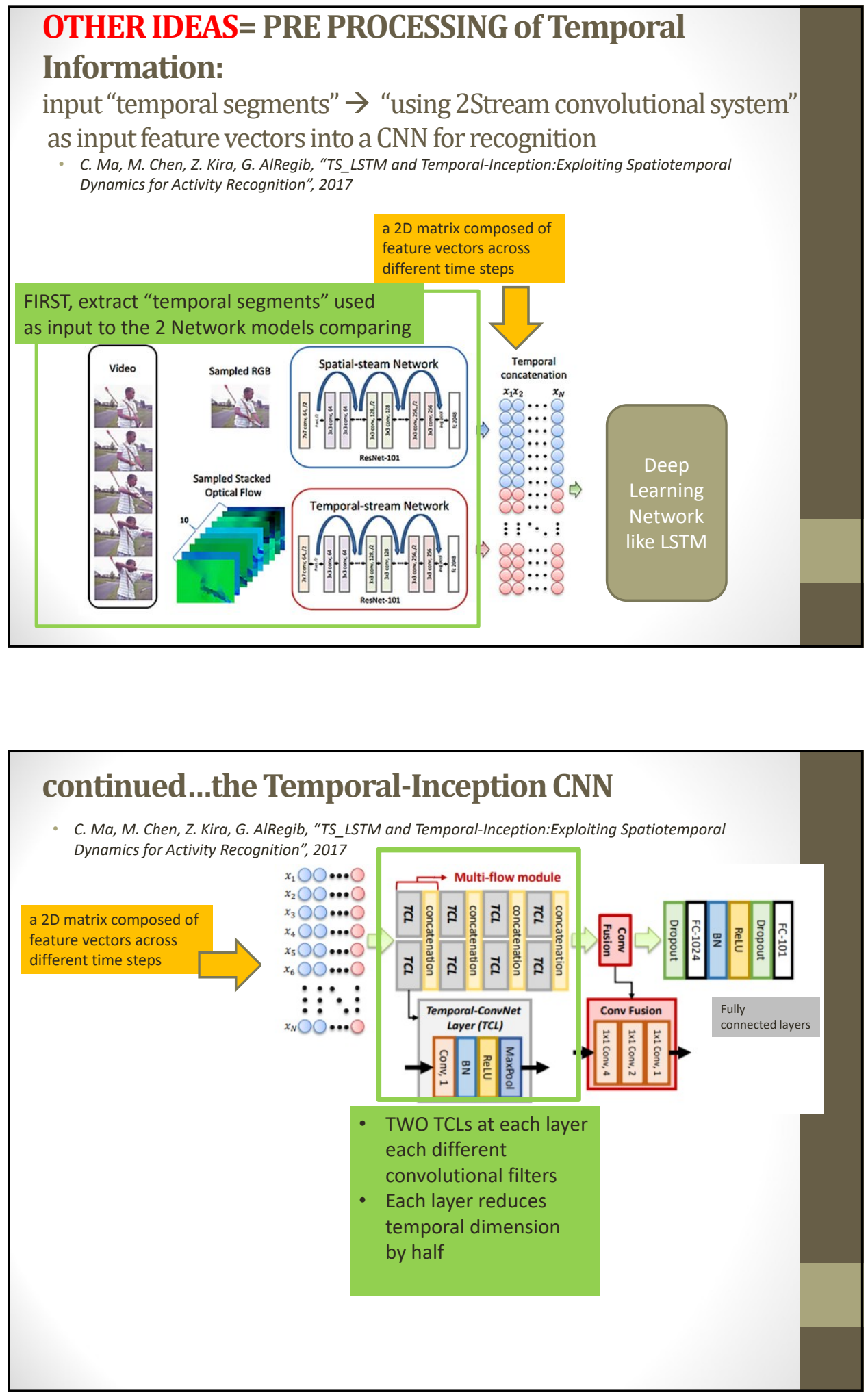
COMPARING: LSTM versus Temporal CNN(inception style)

- Compare Temporal Segment LSTM and Temporal ConvNet as described in C. Ma, M. Chen, Z. Kira, G. AlRegib, "IS_LSTM and TemporalInception:Exploiting Spatiotemporal Dynamics for Activity Recognition", 2017

- Performed almost at the SAME level 94.1 versus 93.9 on UCF101 dataset for
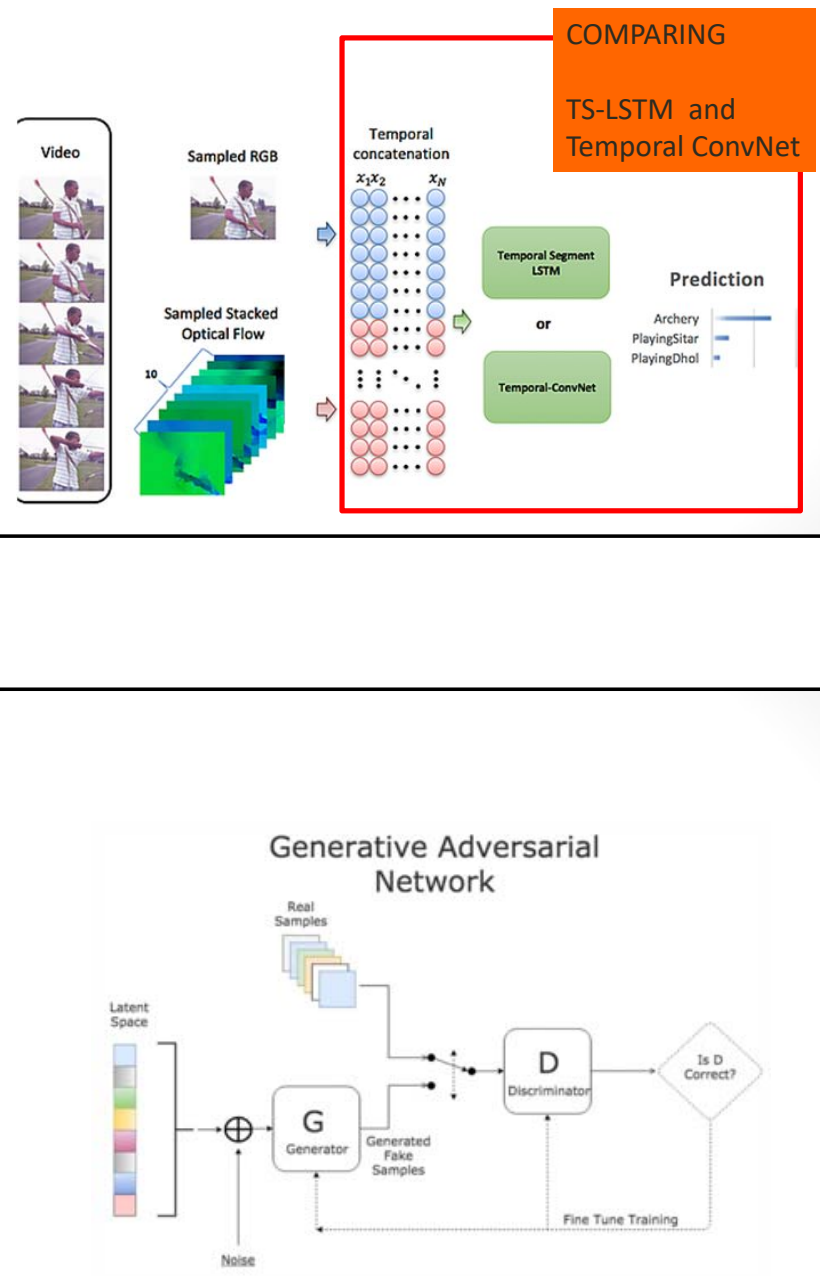

-- network to teach the network

GENERATIVE ADVERSARIAL NETWORKS 


\section{GAN: generative adversarial network}

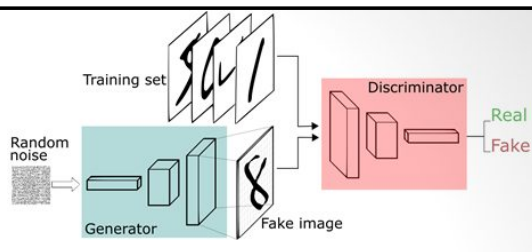

- 2Networks:

- $1^{\text {st }}$ network (“discriminator") $=$

takes the image as input and output is the determination of whether the input is a true representation or fake representation of some class.

- $2^{\text {nd }}$ network ("generator") is trained to generate input to train the first network. The "adversarial" component of this concept is the second network ("generator") tries to progressively create hard input to "fool" the first network.

= zero-sum or minimax two player game

By doing so, ultimately the first network will achieve better discriminating capabilities, meaning higher accuracy

\section{GAN -parking example training}

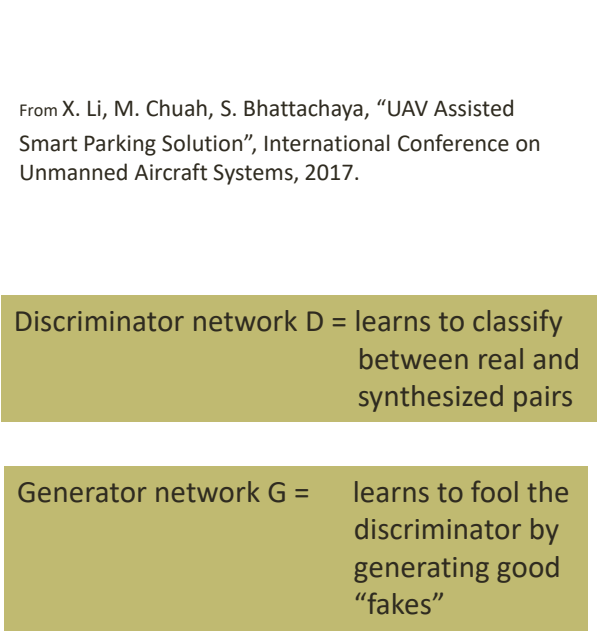

RESULT $\rightarrow$ use the Discriminator network D for empty parking space detection

\section{Negative examples}

Real or fake pair?
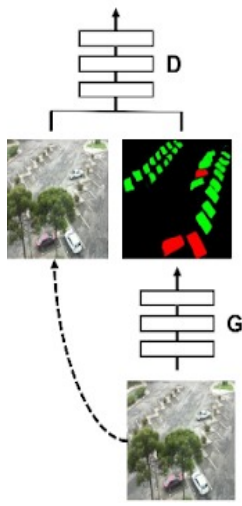

Positive examples

Real or fake pair?

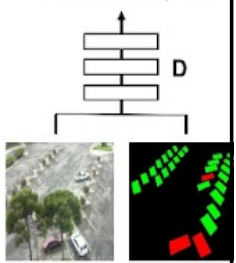

D: Discriminator

G: Generator

During the training process, these two networks are trained simultaneously, and the generator is learning to generate better synthesized pair while the discriminator is learning to telling the difference between the real and synthesized pairs 


\section{GAN -parking example training}

Generator Architecture = Encoder-Decoder Model

From X. Li, M. Chuah, S. Bhattachaya, "UAV Assisted

Smart Parking Solution", International Conference on

Unmanned Aircraft Systems, 2017.

Encoder-Decoder often used for information translation problems, image segmentation,

image synthesis

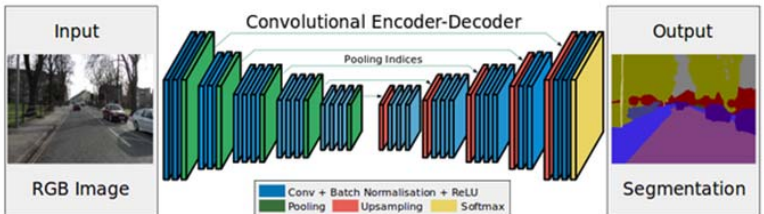

Generator network $\mathrm{G}=$ learns to fool the

Convolutional Encoder + Decoder discriminator by generating good "fakes"

\section{GAN- parking example results}

Cameras mounted aerially -to be
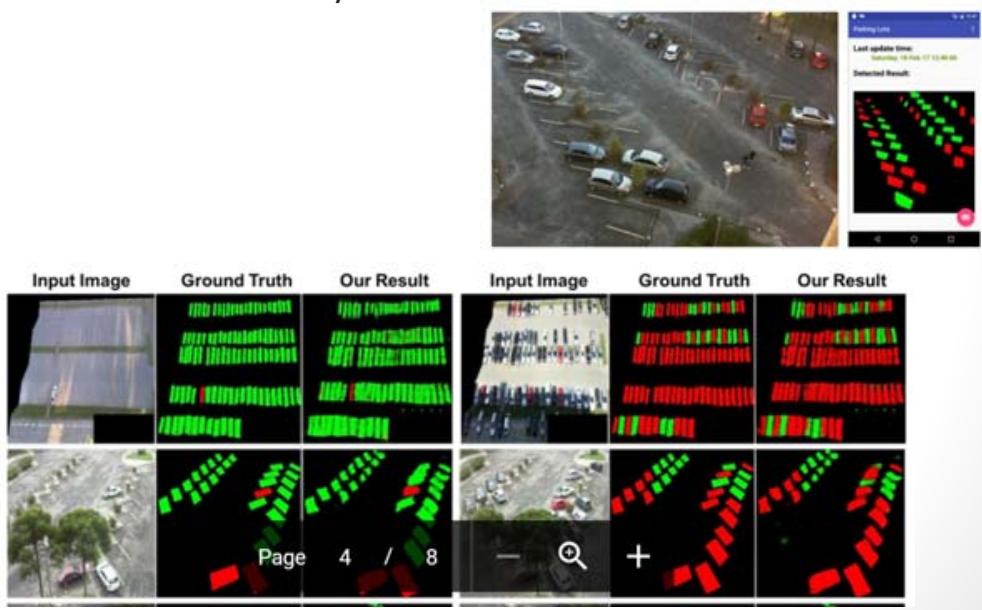


\section{GAN - applications}

APPLICATIONS:

1. may be more suited to future predictive problems such as future video frame predictions

2. discriminator network could be used as a "feature extractor" stage in a more complex CNN.

3. Generator network could be used for synthesis

4. GANs used to produce photorealistic images for modeling scenes, to reconstruct 3D models of objects from images and for texture synthesis [9-11]. They have also been used for various object detection techniques like detection of open spaces for parking [12].

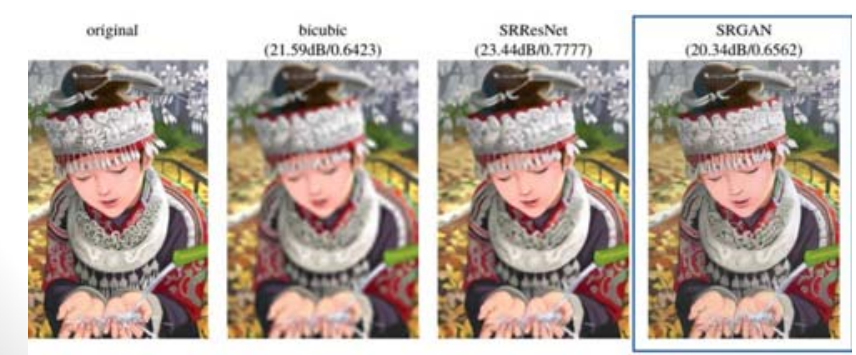

Application:

Resolution

Enhancement

https://www.cs.toronto.edu/ duvenaud/courses/csc2541/slides/gan-applications.pdf

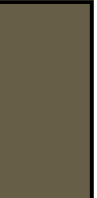




\section{Option 1 -resize data}

- problem of variable sized input propagates down to the first fully connected/inner product layer which requires a vector of fixed size.

- Resize data to expected input size for Network

- SPECIAL NOTE: if there are NO Fully connected layers, you do not need to do this. The output layer will be larger but, it does not represent classes but, feature vectors and can potentially be used in same way as before. One example Encoder-Decoder

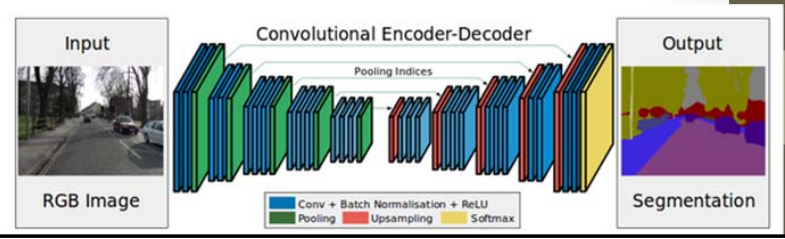

\section{Option 2 - "Spatial Pyramid Pooling"}

- "Spatial Pyramid Pooling in Deep Convolutional Networks for Visual Recognition" by He et al. proposes a Spatial Pyramid Pooling layer.

- propose to add the Spatial Pyramid Pooling Layer just before the first fully-connected layer (details in the paper).

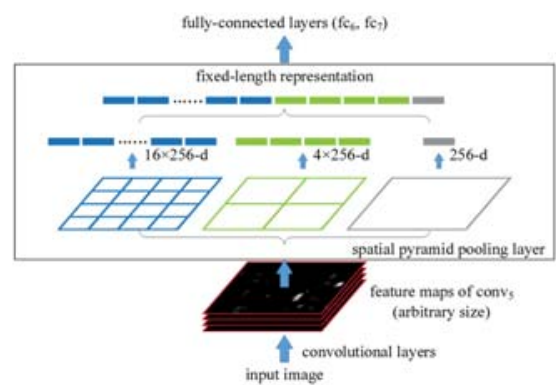

hierarchically partitions the feature maps of the last convolutional layer (or the subsequent pooling or response normalization layer) into a fixed number of bins.

Within these bins, responses are pooled as usually, creating a fixedsized output 


\section{GUIDELINES}
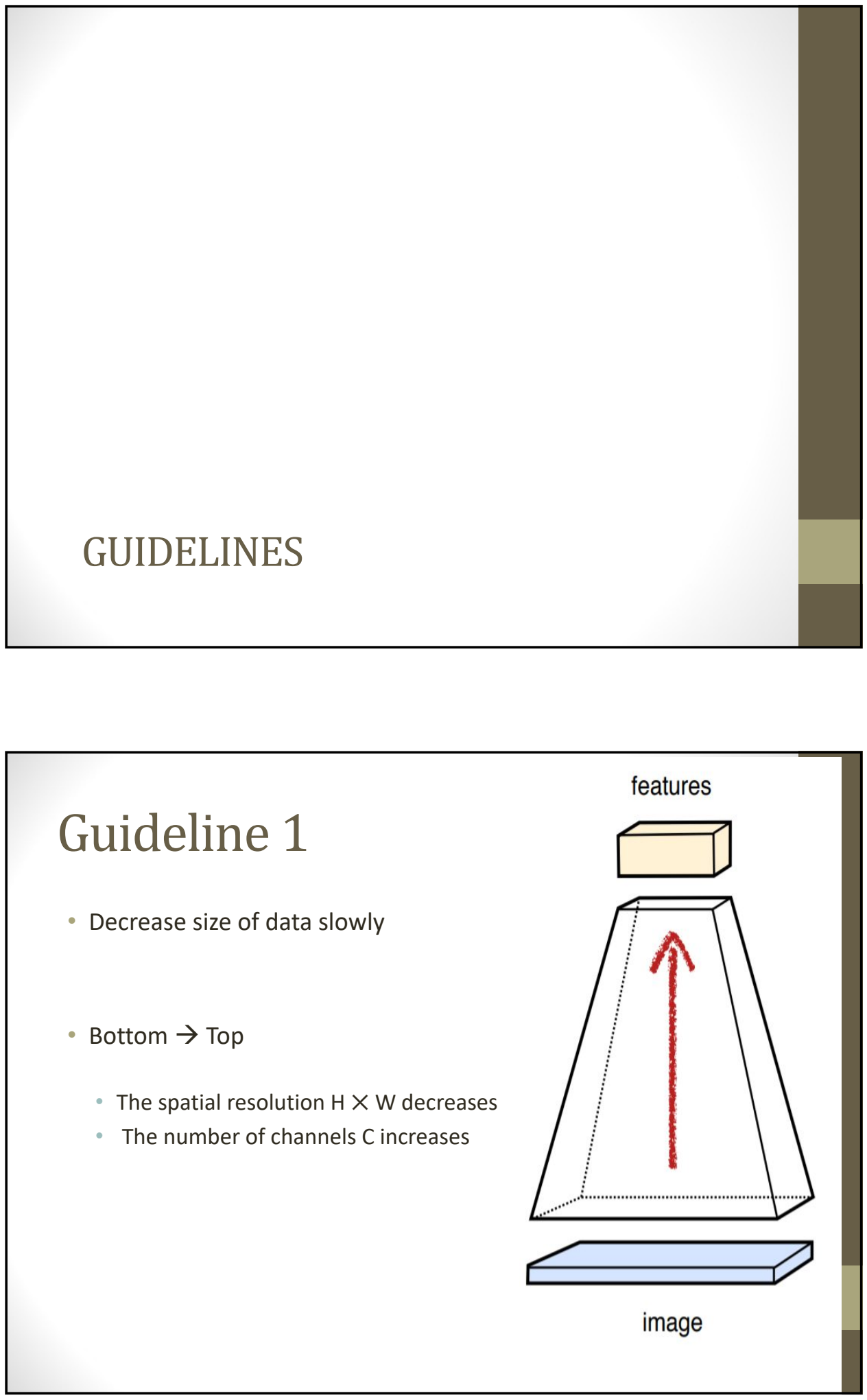

liv

Proc. of SPIE Vol. 10646 1064601-54

Downloaded From: https://www.spiedigitallibrary.org/conference-proceedings-of-spie on 26 Apr 2023 Terms of Use: https://www.spiedigitallibrary.org/terms-of-use 


\section{Guideline 2 - Filters}

"neuron"

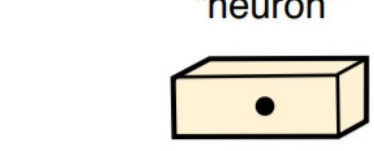

- Receptive field must be large enough to capture objects of interest

- The image region influencing a neuron

- Anything happening outside is invisible to the neuron

- HOW TO INCREASE RECEPTIVE FIELD

1. Large filters

2. Chains of small filters

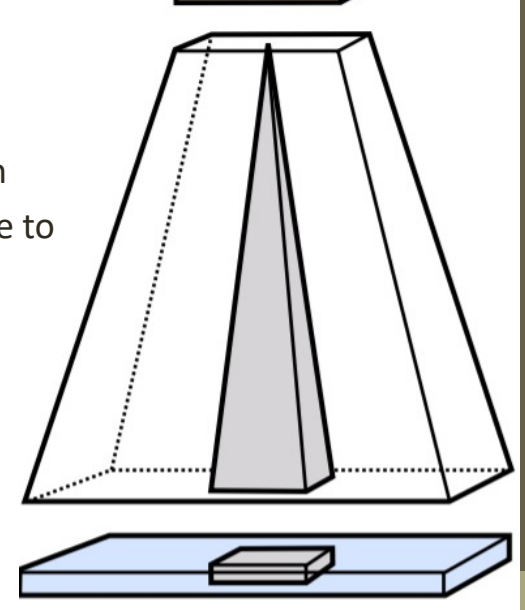

neuron's receptive field

\section{Guideline 3- Filters}

- User chain of smaller filters rather than large filter

- Reduces number of parameters $\rightarrow$ faster

- Get same receptive field as the larger filters

- Get more nonlinearities introduced (example 2 nonlinearities)

One big filter bank

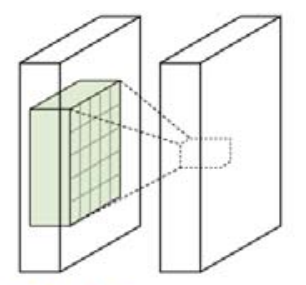

$5 \times 5$ filters

+ ReLU
Two smaller filter banks

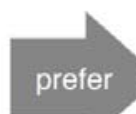

prefer

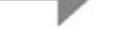

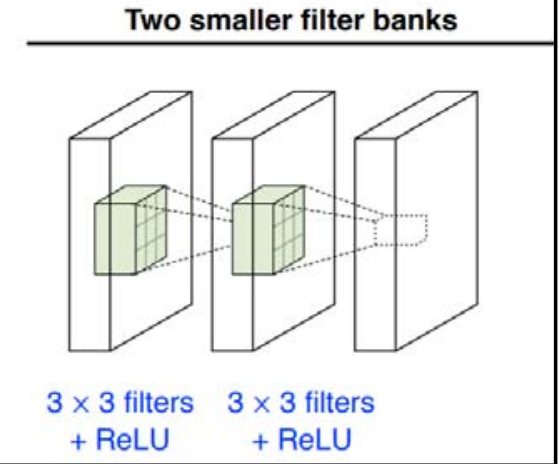




\section{Guideline 4- number of filters in layer (= \# output channels)}

- Be conservative, don't have too many filters (\# filters K below)

$H \times W \times C$

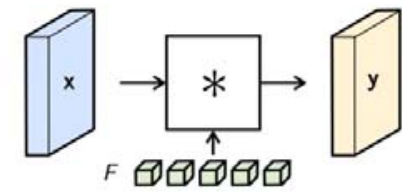

$H_{f} \times W_{f} \times C \times K$

$C=$ num. input channels $\longrightarrow \uparrow$
$K=$ num. output channels
Num. of operations

$\frac{H \times H_{f}}{\text { stride }} \times \frac{W \times W_{f}}{\text { stride }} \times \underline{C \times K}$

Num. of parameters

$H_{f} \times W_{f} \times C \times K$

\section{Guideline 5 - when computation issue consider separable filters}

- Like MobileNet consider separable filters to reduce number of parameters and hence computations. 

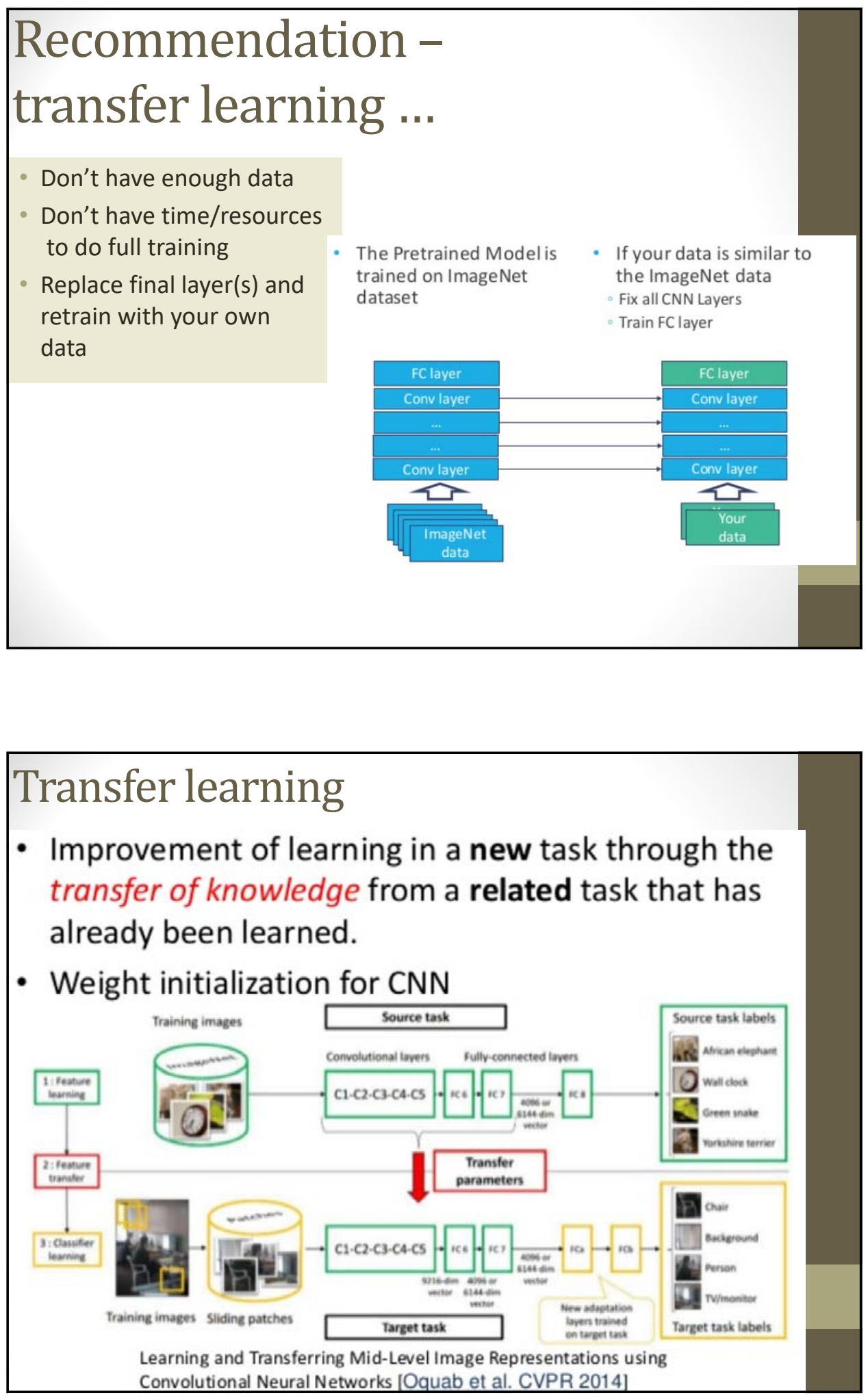


\section{Recommendation Summary}

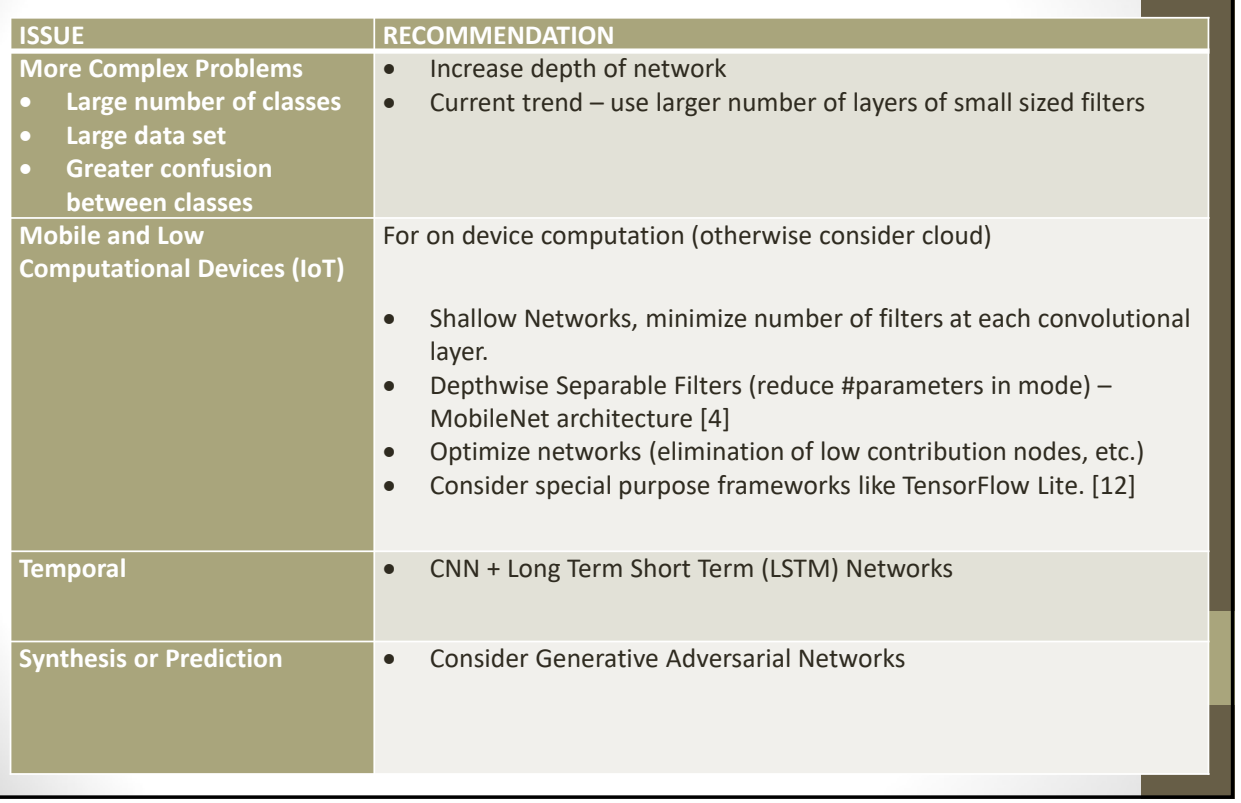

\section{Overall Recommendations}

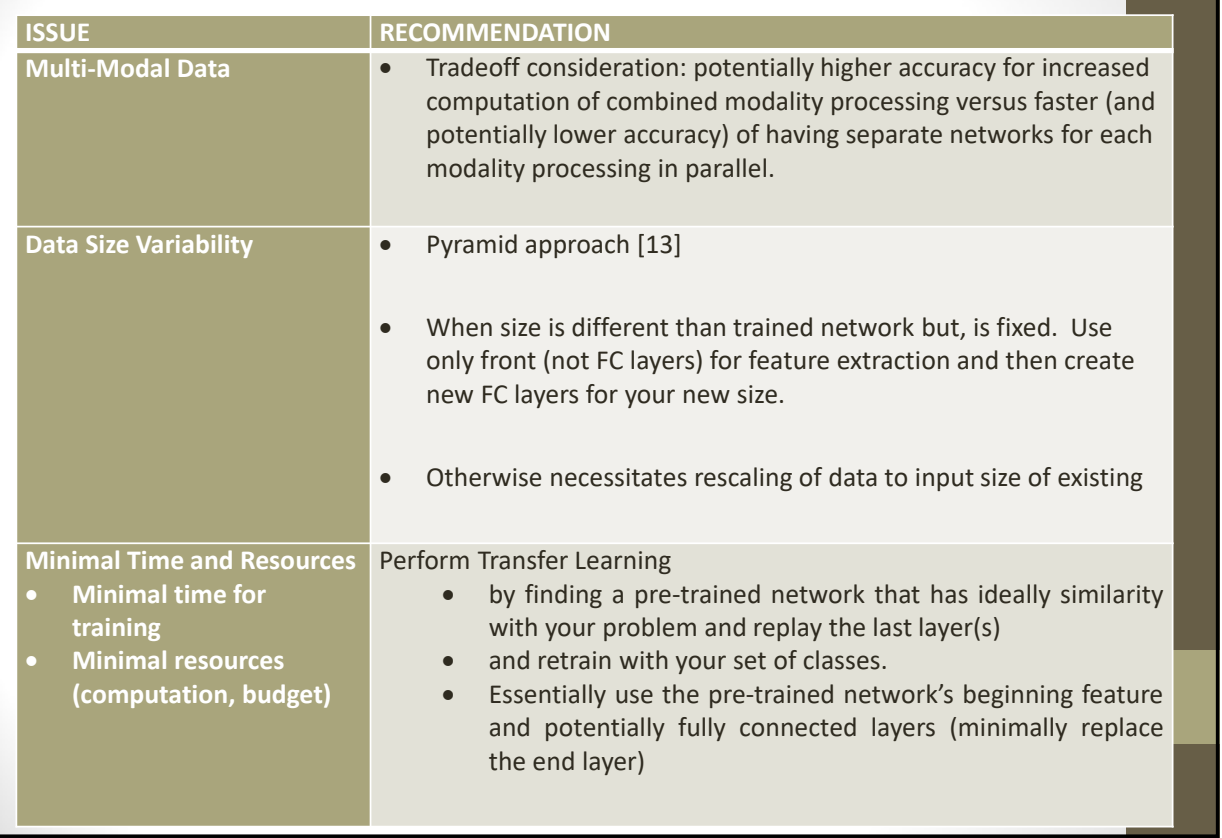




\section{Overall Recommendations}

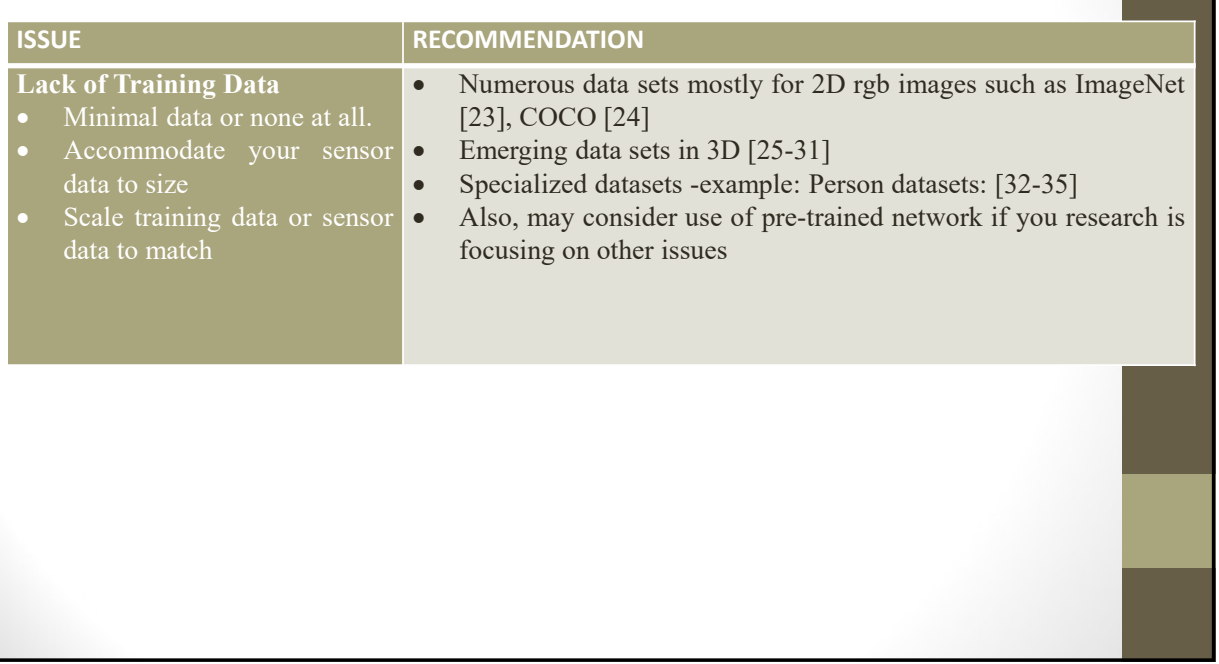

\section{Resources}

Beyond frameworks 


\section{CNN Frameworks}

- Personal preference : TensorFlow $\rightarrow$ new TensorFlow lite for mobile devices using hardware acceleration on Android (android neural network api)

This year ...

Caffe

(UC Berkeley)

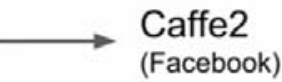

Torch

(NYU / Facebook)

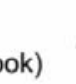

Theano

(U Montreal)

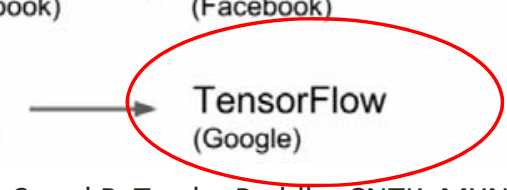

PyTorch

(Facebook)

ensorFlow

Newer: Caffe2 and PyTorch, Paddle, CNTK, MXNet

Suggestion: use TensorFlow or PyTorch
Paddle

(Baidu)

\section{CNTK}

(Microsoft)

MXNet

(Amazon)

\section{Computational Resources}

- Cloud general

- New Google AutoML - As a service (in alpha) https://cloud.google.com/automl/

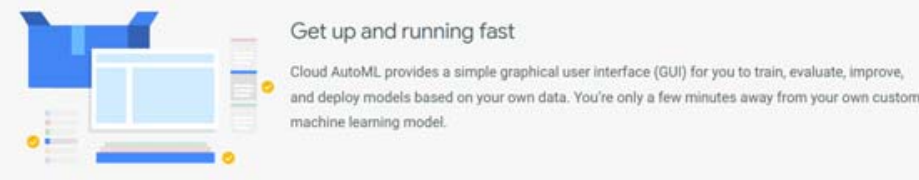

- Cloud Services: Google Machine Learning, Microsoft Cognitive Services

- https://cloud.google.com/ml-engine/

- https://azure.microsoft.com/en-us/services/cognitive-services/

- Intel Movidius Neural Computing Stick --has VPU, speed up Ubuntu laptop w/ USB3 -- supports Caffe Framework and uses Intel's SDK https://movidius.github.io/ncsdk/ 


\section{Datsets that have segmentation ground truth}

- http://host.robots.ox.ac.uk/pascal/VOC/voc2012/

- http://cocodataset.org/\#home

- Example from COCO

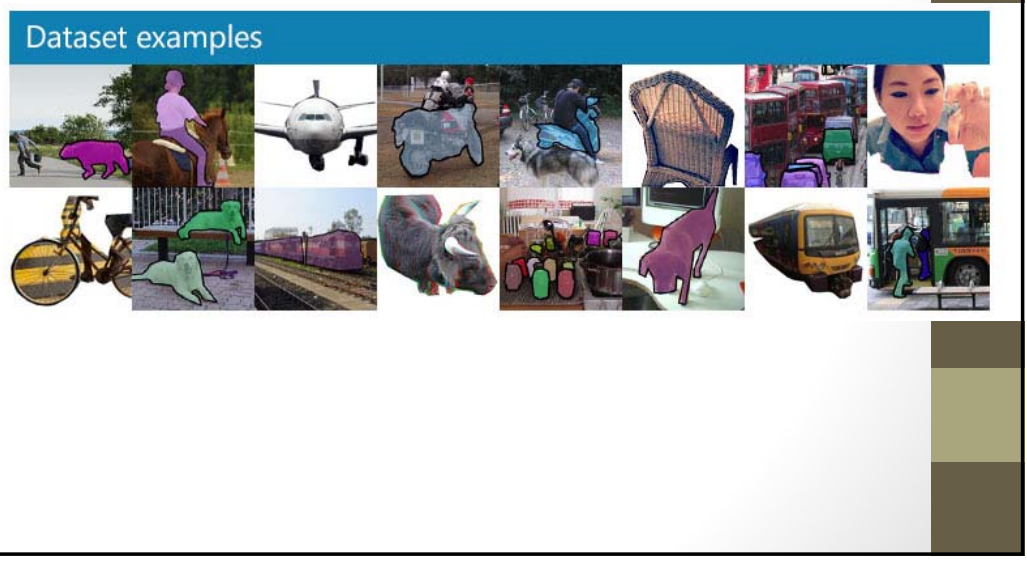

\section{RGB-D datasets}

- Image Net - 14 Million images, 21,841 sub-categories

http://image-net.org

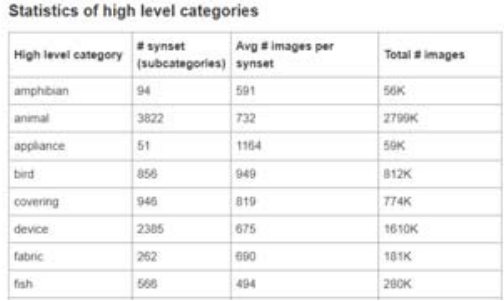

- COCO -100 objects, images $=200 \mathrm{~K}$ labeled, $330 \mathrm{~K}$ total

http://cocodataset.org

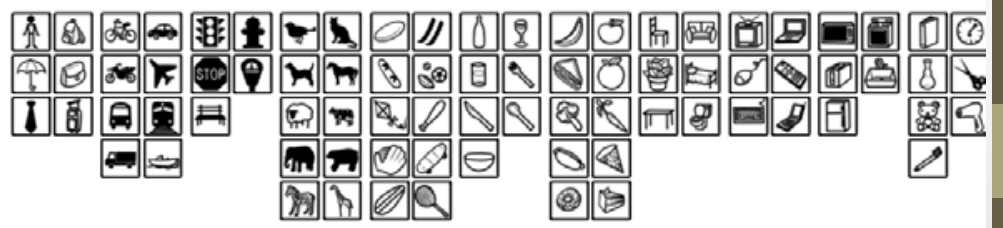




\section{SPECIAL PURPOSE:}

\section{RGB-D People Dataset}

- http://www2.informatik.uni-freiburg.de/ spinello/RGBD-dataset.html

- 3000 images from 3 Vertically mounted Kinect
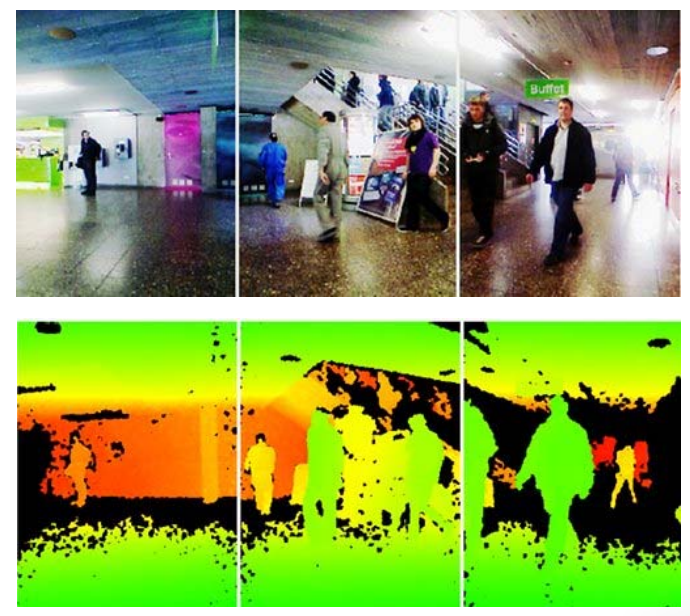

\section{SLR Human attributes dataset}

- Person -rgb +depth (kinect) - 100 people, 100,000 images http://srl.informatik.unifreiburg.de/human attributes dataset
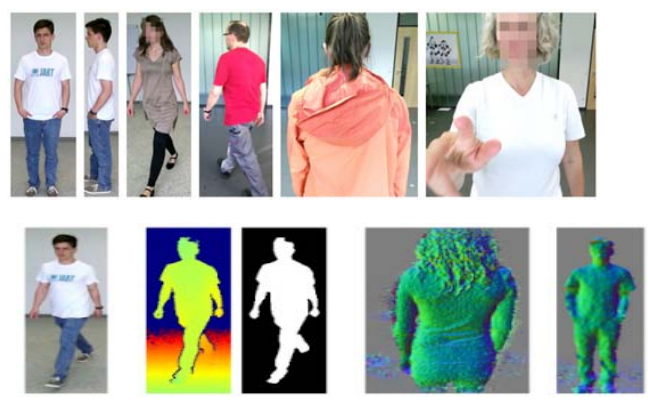

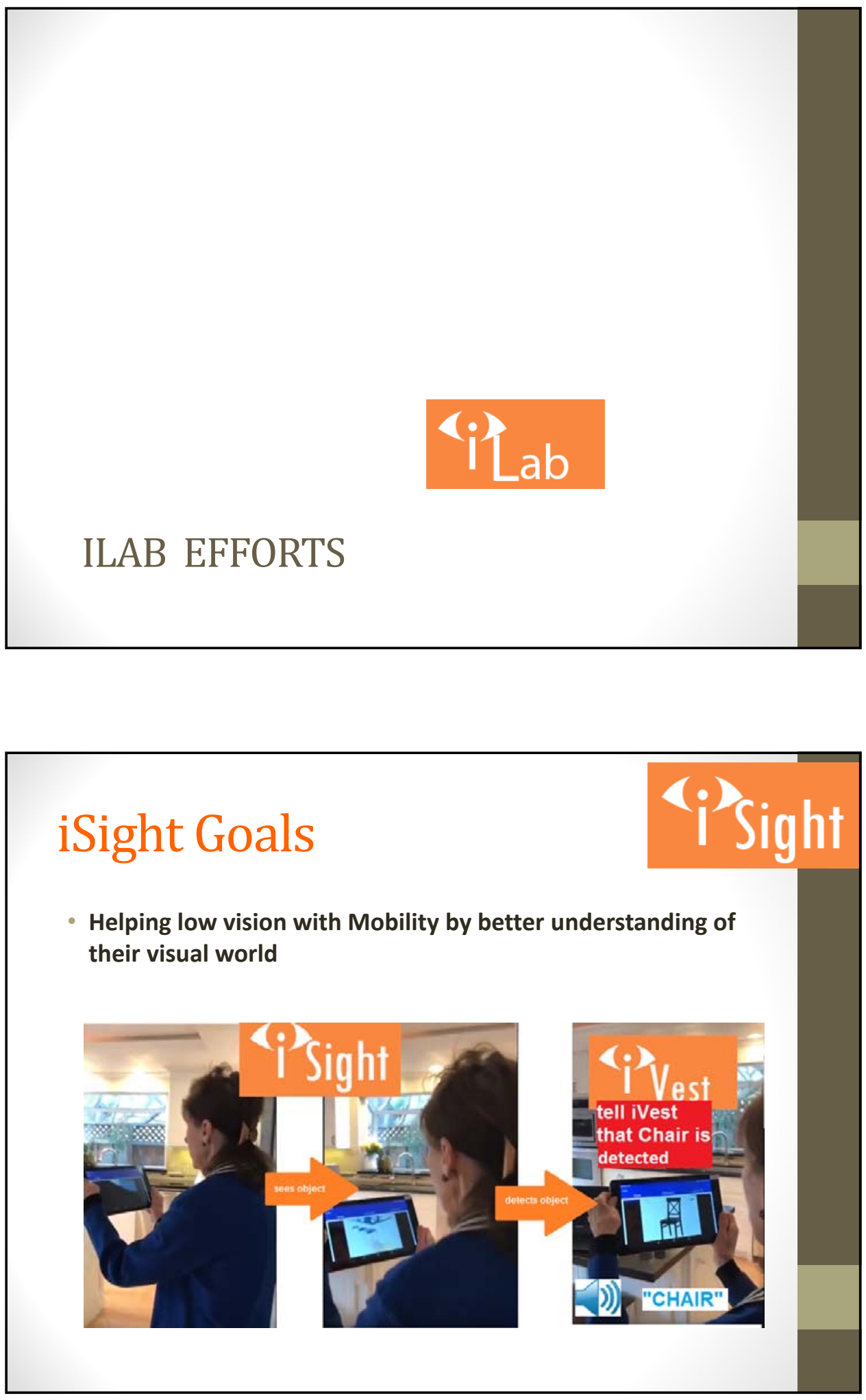


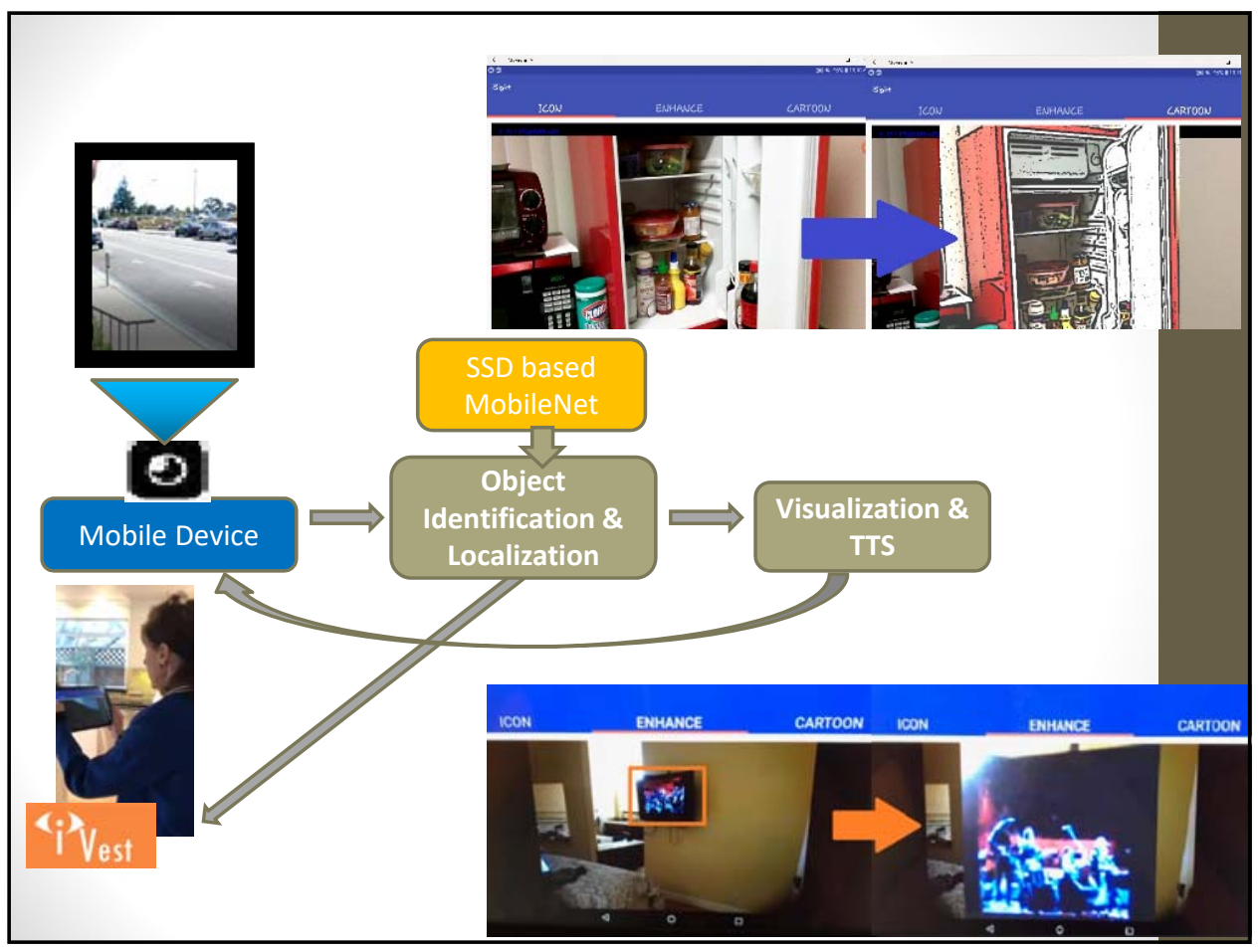

\section{Seeing Eye Drone}

- Replace seeing eye dog

- Computer vision -3D, deep learning

- Obstacle detection and report

- Using Multiple CNN for User Detection and Heading and Obstacle Detection and Selection for Avoidance

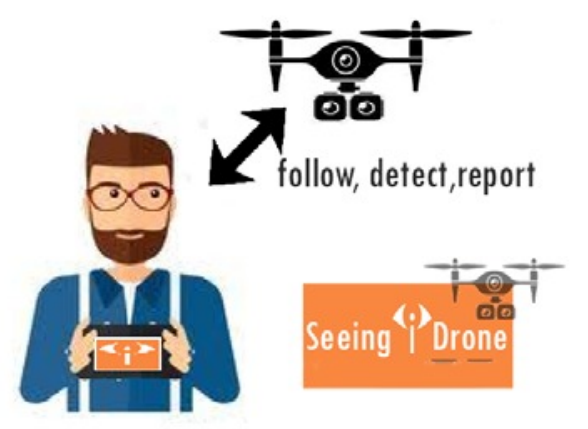




\section{Conclusions}

- Follow Guidelines

- "areas to explore"

- temporal learning

- Gamming the system - GANs

- Multi-modal considerations

- Varying data size

Proc. of SPIE Vol. 10646 1064601-65

Downloaded From: https://www.spiedigitallibrary.org/conference-proceedings-of-spie on 26 Apr 2023 Terms of Use: https://www.spiedigitallibrary.org/terms-of-use 


\title{
Deep Learning and Computer Vision: Guidelines and Special Issues
}

\author{
Lynne Grewe ${ }^{\mathrm{a}}$ and Garrett Stevenson ${ }^{\mathrm{a}}$ \\ ${ }^{a}$ Computer Science, California State University East Bay, 25800 Carlos Bee Boulevard, Hayward, \\ CA USA, 94542
}

\begin{abstract}
The catapult of Computer Vision into recent societal prominence is represented by advancements in self-driving cars, drone autonomy, and cities of the future. Central to these advancements are the developments of Deep Learning with Computer Vision to tackle the important tasks of object classification and localization. This paper surveys some of the current research and presents current guidelines for working in computer vision with deep learning. Additionally, special topics are highlighted including Multi-Modal Vision with Deep Learning and Temporal Networks.
\end{abstract}

Keywords: Deep Learning, Computer Vision, Multi-Modal Deep Learning, Temporal Networks

\section{RESARCH TRENDS}

One Deep Learning research trend is "going deeper", creating CNNs with a greater number of layers. In [1], VGG Net is a CNN with 19 layers (2014). In 2015, GoogLeNet [2], a 22 layer network, was able to achieve a top 5 error rate of $6.7 \%$ and was different than previous CNNs in that is was not a sequentially layered network and instead had parallelly processed layers. At the same time Microsoft ResNet [3] is a 152 layer network that produced an incredibly low error rate of 3.6\% for the ILSVRC 2015 challenge. Generally, more complex problems (larger number of classes) can require having deeper networks to yield higher accuracy.

At the same time that some researchers have gone deeper, there is a strong interest in mobile vision and using CNN/deep learning on the mobile devices which are comparatively low computational devices compared to the machines running ResNet. So, going deep (or as deep) will not work on these devices. MobileNet [4] is an example of recent work that creates a CNN architecture that optimizes the network to run more efficiently on mobile devices by using depth wise separable convolution. Following the MobileNet architecture a general guideline for low computational devices is to stick with shallower networks. In [5], use of MobileNet architecture is shown for the iSight system that uses Deep Learning and Visualization to assist people with Low Vision.

Another recent emerging trend is that of Generative Adversarial Networks [6,7]. With GANs there are two networks, the first network ("discriminator") takes the image as input and output is the determination of whether the input is a true representation or fake representation of some class. At the same time a second network ("generator") is trained that generates input to train the first network. The "adversarial" component of this concept is the second network ("generator") tries to progressively create hard input to "fool" the first network. By doing so, ultimately the first network will achieve better discriminating capabilities, meaning higher accuracy. This can be thought of as a zero-sum or minimax two player game This form of Deep Learning Networks may be more suited to future predictive problems such as future video frame predictions [8] over non-GAN CNNs. Additionally, a GAN discriminator network could be used as a "feature extractor" stage in a more complex CNN. GANs have been used to produce photorealistic images for modeling scenes, to reconstruct 3D models of objects from images and for texture synthesis [9-11]. They have also been used for various object detection techniques like the detection of open spaces for parking [12].

\section{MULTI-MODAL VISION AND DEEP LEARNING}

Multi-modal vision is the idea that more than one kind of data is being presented to the system. This data may come from multiple sensors and may even be different in nature. Multi-modal data processing with a Deep Learning 
framework has not been fully explored but some examples can be found at [13-17]. One technique is to create a CNN only with one sensor data and use the other sensors' data for additional information. For example, in [17] a system is discussed that performs object detection using a CNN with only 2D image data. Subsequently, two depth sensors (one stereo and the other based on IR technology) have collected depth information of the scene and using the detected location in the 2D image of the object, the 3D location of the object can be estimated. The advantages of such a technique is more simplicity and faster processing than using multi-modal data in the CNN.

In [15, 16], 2D image data and 3D depth information is used directly in the CNN. In [16], an exploration of different ways to incorporate it are compared for performance. Having separate CNNs that run in parallel for 2D and 3D is one case. The second case is presenting $2 \mathrm{D}$ and $3 \mathrm{D}$ as a $4 \mathrm{D}$ image input (r,g,b, depth) into a single CNN. These two multimodal systems are compared to a 2D only (single sensor data) system. The best results were achieved for the 4D image input. At the same time, this fused data network will be the largest and hence will potentially run slower than the separate CNN case which could potentially run the 2 separate CNNs in parallel. This indicates that presenting all the data at once to a single CNN may yield superior results. Certainly this approach will let the Network learn how to combine the data at different layers (and scales) to best achieve features to yield higher accuracies.

\section{TEMPORAL NETWORKS}

In addition to looking at different kinds of sensor data, gathering data over time and processing it in a temporal fashion can yield superior results and allow for different applications like activity recognition ("man eating”, "woman walking”) and video captioning ("2 persons walking a dog"). Architectures to incorporate temporal processing in include Recurrent Neural Networks (RNN) and Long Short Term (memory) Networks (LSTM). Recurrent Neural Networks (RNNs) have been successfully applied to processing both speech and video analysis [18] but, are primarily used for speech as they do not inherently capture spatial data as CNNs do. One work looks at integrating CNN and RNN directly in [19]. However, the use of CNNs for temporal processing is best represented in Long Short Term Memory Networks (LSTM) [20-22].

One problem with neural network models using state information is the ability to backpropogate anderro over a longrange temporal span becomes difficult. The LSTM networks have nodes which allow them to propogate without modification using a simple learned gating function and this is a kind of "long term effect" called "long range learning". Additionally, the nodes in a LSTM network allow the network to learn when to "forget" previous hidden states and when to update hidden states given new information. This is a kind of "short-term" memory, basically having an expiration to previous information. In [20], a CNN is used to perform object identification which is fed into an RNN (textual input) to come up with a image caption. More recently in [22], a CNN is used to process the image frames of a video sequence that is then fed into a LSTM to perform activity recognition, and both image and video captioning.

\section{GUIDELINES}

Every vision application has different demands. Some systems will have access to good computational resources and others may need to run on mobile and embedded systems. Some systems will have single sensor data and others multisensor/multi-modal data and so on. Table 1 shows some guidelines for different operating scenarios.

\begin{tabular}{|l|l|}
\hline ISSUE & RECOMMENDATION \\
\hline $\begin{array}{l}\text { More Complex Problems } \\
\bullet \quad \text { Large number of classes }\end{array}$ & $\bullet$ Increase depth of network \\
$\bullet \quad$ Large data set & filters \\
- Greater confusion between classes & $\bullet \quad$ Consider Generative Adversarial Networks \\
\hline $\begin{array}{l}\text { Future Looking } \\
\text { Beyond classification of current state, looking to } \\
\text { predict future }\end{array}$ & \\
\hline
\end{tabular}




\begin{tabular}{|c|c|}
\hline Mobile and Low Computational Devices (IoT) & $\begin{array}{cl}\text { For on device computation (otherwise consider cloud0 } \\
\text { - } & \text { Shallow Networks } \\
\text { - } & \text { Depthwise Separable Filters (reduce \#parameters } \\
\text { - } & \text { Optimize - MobileNet architecture [4] } \\
& \text { contribution norks (elimination of low } \\
\text { - } & \text { Consider special purpose frameworks like } \\
& \text { TensorFlow Lite. [13] } \\
\end{array}$ \\
\hline Multi-Modal Data & $\begin{array}{l}\text { - Tradeoff: potentially higher accuracy for } \\
\text { increased computation of combined modality } \\
\text { processing versus faster (and potentially lower } \\
\text { accuracy) of having separate networks for each } \\
\text { modality processing in parallel. }\end{array}$ \\
\hline Data Size Variability & $\begin{array}{l}\text { Pyramid approach [14] } \\
\text { - When size is different than trained network but, is } \\
\text { fixed. Use only front (not FC layers) for feature } \\
\text { extraction and then create new FC layers for your } \\
\text { new size. } \\
\text { - Otherwise necessitates rescaling of data to input } \\
\text { size of existing }\end{array}$ \\
\hline $\begin{array}{ll}\text { Minimal Time and Resources } \\
\text { - } \quad \text { Minimal time for training } \\
\text { - } \quad \text { Minimal resources (computation, budget) }\end{array}$ & $\begin{array}{l}\text { - Perform Transfer Learning -by finding a pre- } \\
\text { trained network that has ideally similarity with } \\
\text { your problem and replay the last layer(s) and } \\
\text { retrain with your set of classes. Essentially use } \\
\text { the pre-trained network's beginning feature and } \\
\text { potentially fully connected layers (minimally } \\
\text { replace the end layer) }\end{array}$ \\
\hline $\begin{array}{l}\text { Temporal Processing Needed } \\
\text { • Video applications, time sequence data }\end{array}$ & $\begin{array}{llll}\text { - Use Long Short Term (memory) } & \text { Network } \\
\text { (LSTM) (or CNN+RNN) } & & \\
\end{array}$ \\
\hline $\begin{array}{l}\text { Lack of Training Data } \\
\text { - } \quad \text { Minimal data or none at all. } \\
\text { - } \\
\text { - }\end{array}$ & $\begin{array}{ll}\text { - } & \text { Numerous data sets mostly for 2D rgb images } \\
\text { - } & \text { Emch as ImageNet [23], COCO [24] } \\
\text { - } & \text { Specialized datasets -example: Person datasets: } \\
& \text { [32-35] }\end{array}$ \\
\hline
\end{tabular}

Table 1: Current Deep Learning Recommended Guidelines based on Research Trends.

\section{REFERENCES}

[1] K. Simonyan, A. Zisserman, "Very Deep Convolutional Networks for Large-Scale Image Recognition”, ICLR, 2015

[2] C. Szegedy, W.Liu, Y. Jia, et al, “Going Deeper with Convolutions”, CVPR, pp 1-9, 2015.

[3] K. He, X. Zhang, S. Ren and J. Sun, "Deep residual learning for image recognition”, CVPR, 2016.

[4] A. Howard, M. Zhu, B. Chen, D. Kalenichenko, W. Wang, T. Weyand, M. Andreetto, H. Adam, "MobileNets: Efficient Convolutional Neural Networks for Mobile Vision Applications”, Computer Vision Pattern Recognition, 2017.

[5] L. Grewe, A. Kashyap, K. Chandran, A. Shahshahani, J. Shahshahani “ iSight: computer vision based system to assist low vision”, SPIE DSS, 2018.

[6] I. Goodfellow, J. Pouget-Abadie, M. Mirza, et. al., “Generative Adversarial Nets”, Machine Learning, 2014.

[7] T. Salimans, I. Goddfellow, et. al, "Improved Techniques for Training GANs", Computer Vision and Pattern Recognition, 2016. 
[8] M. Mathier, C. Couprie, Y. LeCun, “Deep Multi-scale Video Prediction Beyond Mean Square Error”, Computer Vision and Pattern Recognition, 2015.

[9] C. Vondrick, H. Pirsiavash, A. Toralba, "Generating Videos with Scene Dynamics”, Neural Information Processing Systems, 2016.

[10] J. Wu, Y. Wang, T. Xue, X. Sun, W. Freeman, J. Tenebaum, "MarrNet: 3D Shape Reconstruction via 2.5D Sketches”, Neural Information Processing Systems, 2017

[11] M. Sajjadi, B. Scholkopf, M. Hirsch, "EnhanceNet: Single Image Super-Resolution Through Automated Texture Synthesis", Computer Vision and Pattern Recognition, 2017

[12] X. Li, M. Chuah, S. Bhattachaya, "UAV Assisted Smart Parking Solution”, International Conference on Unmanned Aircraft Systems, 2017.

[13] Tensorflow Lite (for mobile and embedded systems), https://www.tensorflow.org/mobile/tflite/, 2018.

[14]K. He, X. Zhang, S. Ren, J. Sun, "Spatial Pyramid Pooling in Deep Convolutional Networks for Visual Recognition”, European Conference on Computer Vision, 2014.

[15]B. Chen, R. Sahdev, J. Tsotsos, "Integrating stereo vision with a CNN tracker for a person-following robot", International Conference Computer Vision Systems, 2017.

[16]L. Grewe, G. Steveson, "Seeing Eye Drone - Deep Learning with 2D and 3D data for Low Vision/Blind Assistance”, pre publication, http://www.mcs.csueastbay.edu/ grewe/ilab/SeeingEyeDrone.html

[17] J. Rizzo, Y. Phan, T. Hudson, E. Wong, Y. Fang "Sensor fusion for ecologically valid obstacle identification: building a comprehensive technology platform for the visually impaired”, International Conference on Modeling, Simulation and Applied Optimization, 2017.

[18] Y. Du, W. Wang, L. Wang, "Hierarchical recurrent neural network for skeleton based action recognition," in Proc. IEEE Conference on Computer Vision and Pattern Recognition, pp. 1110-1118, 2015.

[19] J. Wang, Y.Yang, Z. Huang, C. Huang, W. Xu, “CNN-RNN: A Unified Framework for Multi-Label Image Classification”, CVPR 2106.

[20] O. Vinyals, A. Toshev, S. Bengio, D. Erhan, “Show and Tell: A Neural Image Caption Generator”, CVPR, 2015.

[21] W. Zhu, C. Lan, J. Xing, W. Zheng, Y. Li, L. Shen, X. Xie, "Co-occurrence feature learning for skeleton based action recognition using regularized deep LSTM networks”, Conference on AI, pp. 3697-3704, 2016.

[22] J. Donahue, L. Hendricks, M. Rohrbach, S. Venugopalan, S> Guadarrama, K. Saenko, T. Darrell, "Long-Term Recurrent Convolutional Networks for Visual Recognition and Description”, CVPR, 2017.

[23] ImageNet, http://www.image-net.org/

[24] COCO - common object dataset, http://cocodataset.org,

[25] NYU-Depth V2 dataset, https://cs.nyu.edu/ silberman/datasets/nyu_depth_v2.html

[26] RGB-D (Kinect) Object Dataset, https://rgbd-dataset.cs.washington.edu/

[27] RGB-D SLAM dataset , https://vision.in.tum.de/data/datasets/rgbd-dataset

[28] SUN RGB-D , http://rgbd.cs.princeton.edu/

[29]Big Bird Dataset, http://rll.berkeley.edu/bigbird/

[30]B3DO Dataset, http://kinectdata.com/

[31] A Large Dataset of Object Scans, http://redwood-data.org/3dscan/

[32] RGB-D Person Re-identification Dataset, https://old.iit.it/en/datasets-and-code/datasets/rgbdid.html

[33] UPCV action dataset - http://www.upcv.upatras.gr/personal/kastaniotis/datasets.html

[34] SRL Human Attributes Dataset- http://srl.informatik.uni-freiburg.de/human_attributes dataset

[35] RGB-D People dataset - http://www2.informatik.uni-freiburg.de/ spinello/RGBD-dataset.html 

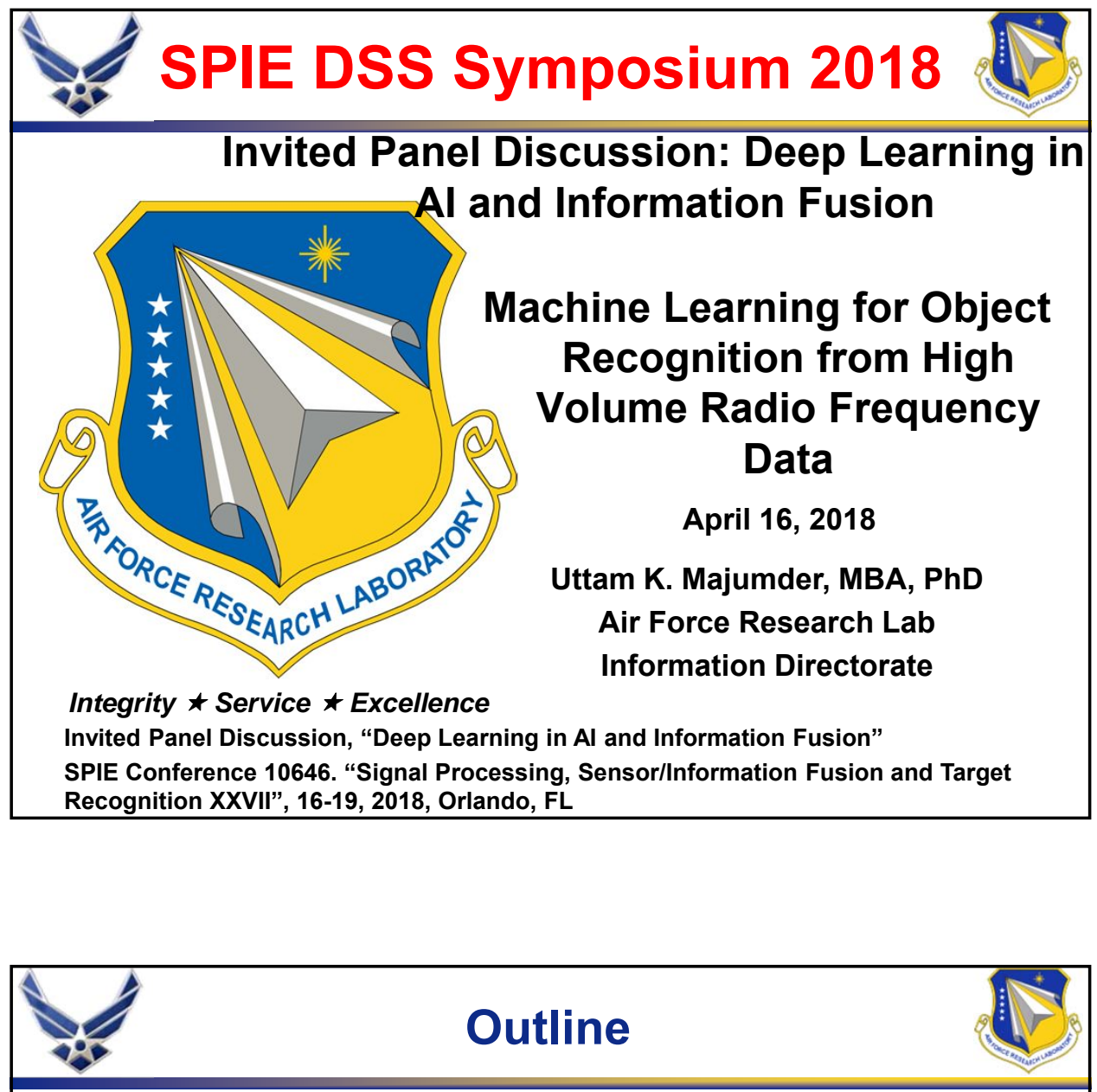

- Artificial Intelligence

- Radio Frequency Data

- Big Data

- Research on Big Data

- High Performance Computing (HPC)

- GPU Enabled Target Classification from SAR Imagery

- Summary 


\section{Introduction to AI}

- $\mathbf{A I}$ - Machines to think/behave/react - ANN

- ML - Data for (Machines) to learn - RL, BN, ILP

- DL - Brain-Inspired NN for robust methods - CNN, RNN

- (mostly supervised from labeled data)

Al: Artificial Intelligence ANN: Artificial Neural Networks

$R L$ : Reinforcement Learning

BN: Bayesian Networks

ILP: Inductive Logic Programming

CNN: Convolutional Neural

Networks

RNN: Recurrent Neural Networks

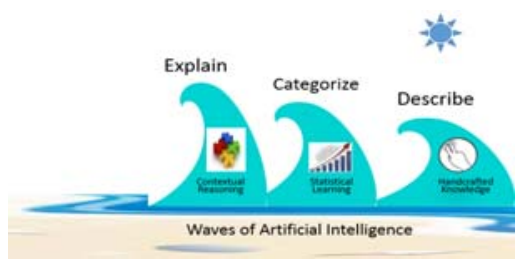

Reference: Andrew Fogg, A history of Deep

Learning, (import.io)

\section{Three Waves of Artificial Intelligence}

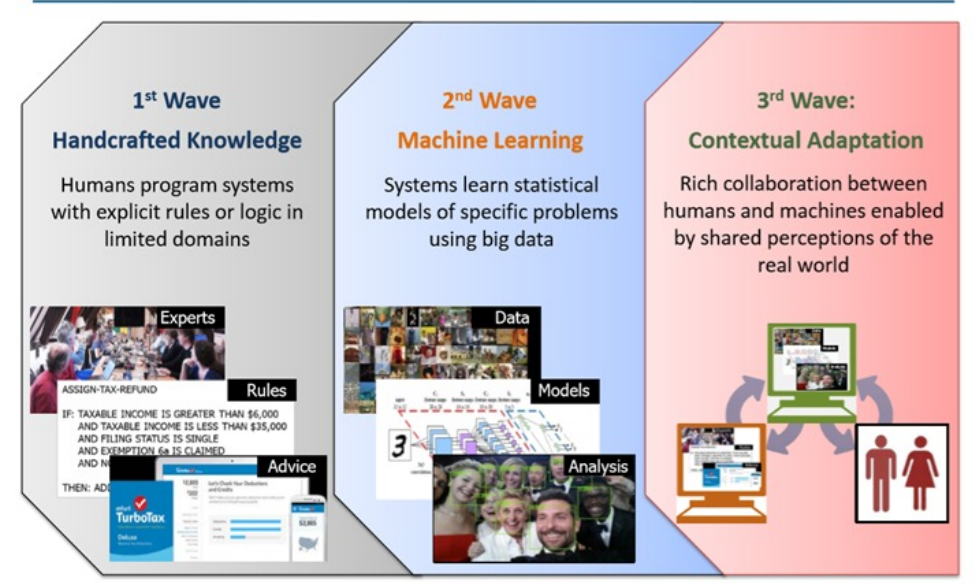

Reference:

DARPA Public Release: RF Machine Learning Systems (RFMLS) Industry Day https://www.darpa.mil/attachments/RFMLSIndustryDaypublicreleaseapproved. pdf 

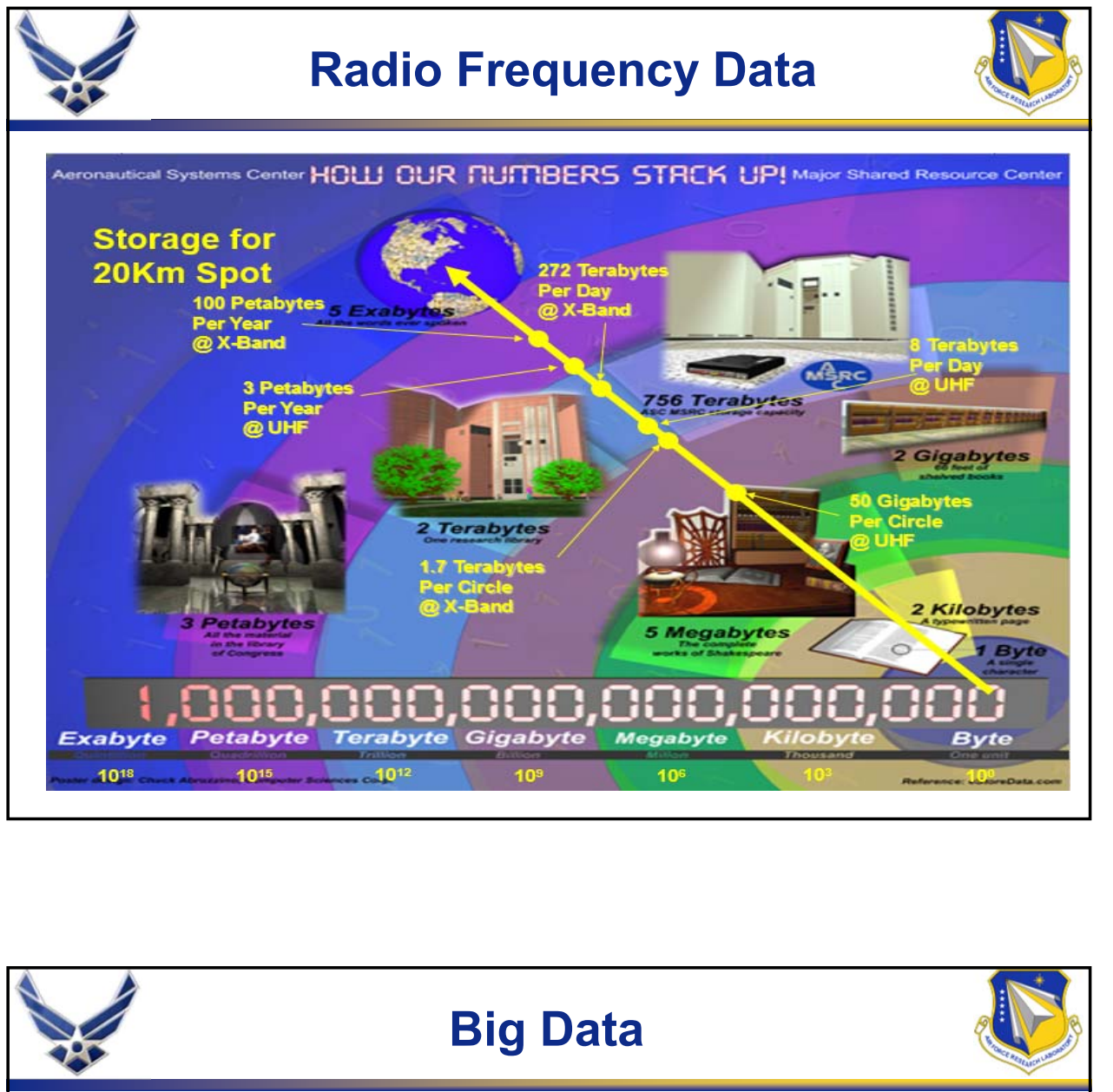

\section{IBM's Definition of Big Data}

https://www.slideshare.net/EdurekalN/introduction-to-big-data-hadoop-i

Ixxii 


\section{Research On Big Data}

- Operational deployment considerations, computation efficiency (SWaP-C)

- The need for HPC for real-time computing

- Model fidelity complimented with data collections for syntheticmeasured data analysis

- Transfer Learning over operating spaces (range, resolution, target settings)

- Big data (volume, velocity, veracity, variety) collaboration policies - what data are accessible for analytics

- Robust evaluation: Validation, Verification, for reproducible results

\section{The Need for Real-time Computing}

$\rightarrow$ In 90's, Machine Learning such as Neural Networks was less popular due to various Tech Barriers and Needs

- Computational Resources were Scarce and Expensive

Limited Sensors or Digitized Business Data to be Analyzed

$\checkmark$ Today, computational resources are not as expensive as in the past; however, abundant of Sensors and Business data needs to be analyzed in Real-time

$\checkmark$ HPC Enables $M L$ algorithm based decision making in real-time or near real-time 


\section{The Advent of HPC}

- Since Late 90's, Computing Technology Has Advanced in an Astounding Pace (The Moore's Law)

- We are Living in the Age of HPC

$>$ Faster memory, CPU, I/O communication, and storage as well as compact/smaller size

$>$ Multi-core Computers

$>$ Graphics Processing Units

Energy-efficient/low-power computing devices (IBM's TrueNorth)

- More to come

Memristor Devices

Specialized Chip/cores for Sparse Graph Processing

\section{$\$$ Recent HPC Hardware Used for} ML Algorithms

\section{CPU}

Few, fast cores $(1-16)$

Good at sequential processing

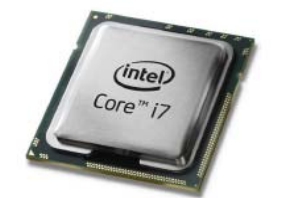

\section{GPU}

Many, slower cores (thousands)

Originally for graphics

Good at parallel computation

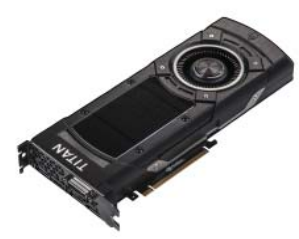

IBM's TrueNorth

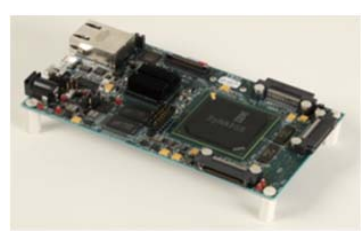

FPGA

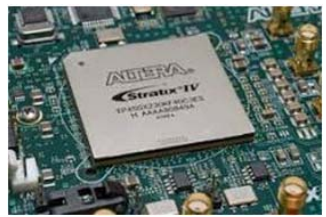




\section{GPU Enabled Target Classification Measured SAR Data}

- Training, validation, and testing data come from the MSTAR* program sponsored by DARPA and the AFRL in the 1990s

- 10 target classes with images taken at various angles

- 15 Degree Elevation Angle dataset for training, 17 Degree dataset for testing

- Roughly 250 images per target class, per angle

- Generally considered an incredibly small dataset for a deep learning application

- Using a single GPU at AFRL/RI HPC

* MSTAR: Moving and Stationary Target Acquisition and Recognition

\section{Target types}

Table 1. The number of images of each object at different depression angles.

\begin{tabular}{ccccccccccc}
\hline Targets & BMP2 & BTR70 & T72 & BTR60 & 2S1 & BRDM2 & D7 & T62 & ZIL131 & ZSU234 \\
\hline $17^{\circ}$ & 233 & 233 & 232 & 256 & 299 & 298 & 299 & 299 & 299 & 299 \\
$15^{\circ}$ & 587 & 196 & 582 & 195 & 274 & 274 & 274 & 273 & 274 & 274 \\
\hline
\end{tabular}
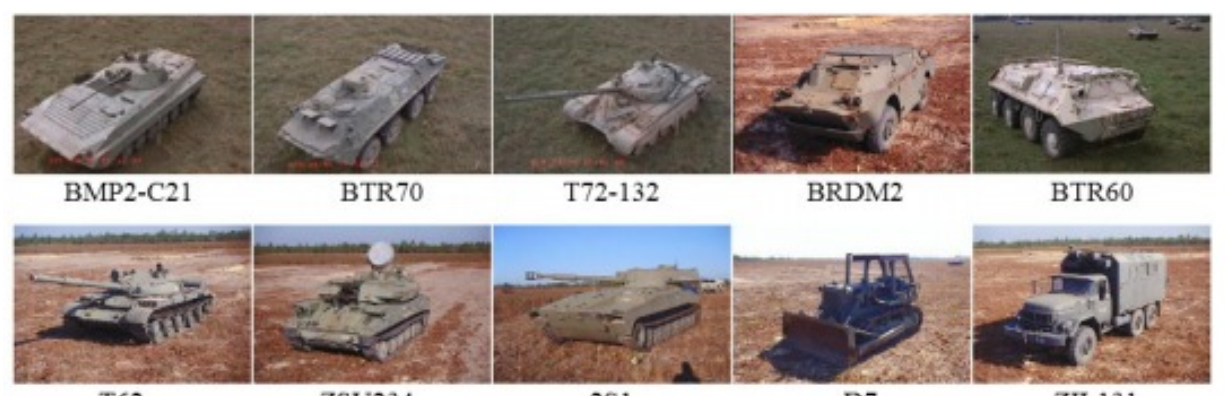


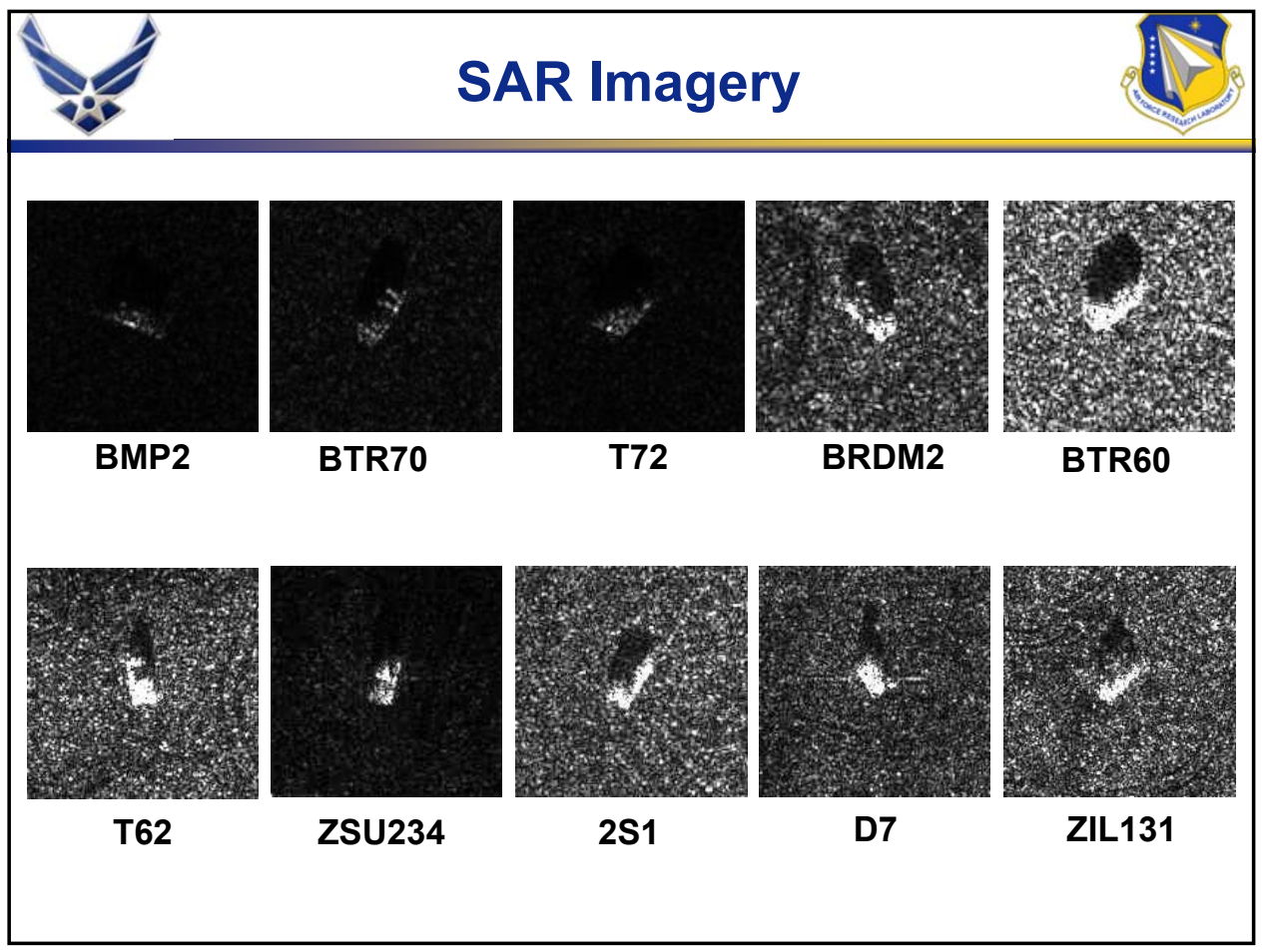

\section{Software Tools}

- Python - Data augmentation methods

- Caffe - Deep learning framework employed via DIGITS and command line

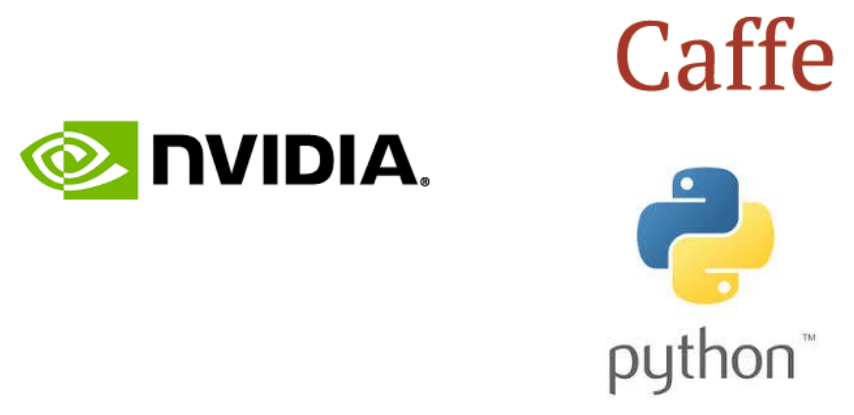




\section{Caffe}

- Deep Learning framework developed by the Berkeley Vision and Learning Center (BVLC)

- Written in highly optimized C++/CUDA code

- Easily define network architectures

- Modify DL models as needed for an application

Convert data and labels to LMDB* format

Train model in Caffe using training dataset

Save learned weights

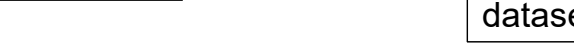

Test model in Caffe using test dataset

* LMDB: Lightning Memory-Mapped Database Manager 


\section{Clean training run}

Neural Net reaches over $\mathbf{9 9 \%}$ accuracy on validation set

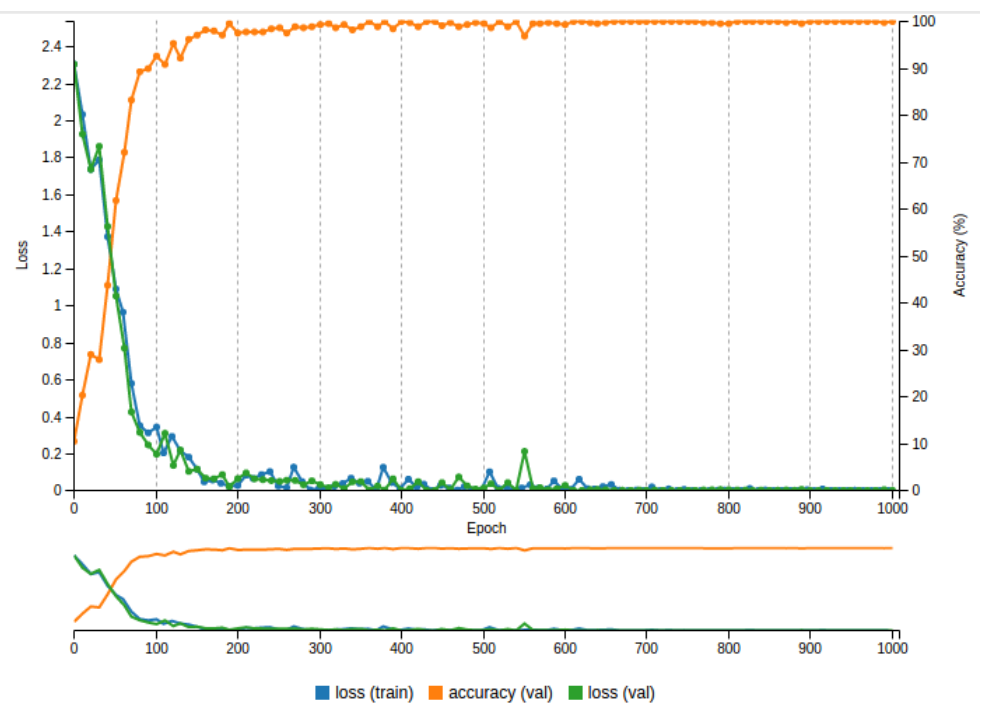

\section{Classification results on Measured Data}

\section{$\sim 99 \%$ accuracy on 10-target classification using Caffe \\ State-of-the-art results}

\begin{tabular}{|c|c|c|}
\hline $\begin{array}{l}\text { tam@uttam-Lenovo-Edge } \\
\text { eights snapshot_iter_ }\end{array}$ & $\begin{array}{l}\text { 80: } \sim \text { caffes } \cdot \text { /bui } \\
\text {. caffemodel }- \text { gpu= }\end{array}$ & $\begin{array}{l}\text { ols/caffe test -model tra } \\
\text { erations }=500\end{array}$ \\
\hline I0815 12:03:19.780280 & 3181 caffe.cpp:300] & Batch 996, accuracy $=1$ \\
\hline I0815 12:03:19.780316 & 3181 caffe.cpp:300] & Batch 996, loss $=0.0115048$ \\
\hline I0815 $12: 03: 19.810564$ & 3181 caffe.cpp:300] & Batch 997, accuracy $=1$ \\
\hline I0815 $12: 03: 19.810609$ & 3181 caffe.cpp:300] & Batch 997, loss $=0.0182748$ \\
\hline [0815 12:03:19.840967 & 3181 caffe.cpp:300] & Batch 998 , accuracy $=0.953125$ \\
\hline [0815 12:03:19.841004 & 3181 caffe.cpp:300] & Batch 998, loss $=0.154708$ \\
\hline I0815 12:03:19.871201 & 3181 caffe.cpp:300] & ccuracy = 1 \\
\hline I0815 12:03:19.871237 & 3181 caffe.cpp:300] & Batch 999, loss $=0.000220068$ \\
\hline I0815 $12: 03: 19.871246$ & 3181 caffe.cpp:305] & Loss: 0.115863 \\
\hline I0815 $12: 03: 19.871261$ & 3181 caffe.cpp:317] & accuracy $=0.989016$ \\
\hline I0815 $12: 03: 19.871305$ & 3181 caffe.cpp:317] & loss $=0.115863(* 1=0.115863$ loss $)$ \\
\hline
\end{tabular}

\section{Key network parameters}

Learning rate 0.001

Batch size 64

1000 training epochs
5 Convolution layers

3 InnerProduct (FC) layers

$2 \times 2$ stride 1 max pool filters
Dropout regularization

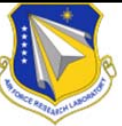




\section{Target Classification Using DNN on}

Synthetic SAR Data

- Training, validation, and testing data used from Synthetic Radar Data

- 30 target classes with images taken at various elevation angles and a single azimuth angle

- Instead of Backprojection Image formation, we used Range-Doppler Map of the Targets

- We found about $99 \%$ accuracy on Target classification

\section{Target Classification Using DNN on Synthetic and Measured SAR Data}

- The objective of this research is to evaluate performance of target classification using Synthetic vs. Measured SAR data ( or vice versa) and identifying the "Gap/Tech Challenges" to generate High Fidelity Synthetic SAR data

- We implemented Training on measured SAR data for three targets and Tested on Synthetic SAR data (of the same targets)

- We found very low accuracy on Target classification

- This is due to the fact that quality (i.e. NIIRS) of synthetic data must be very close to measured data

- This will require huge HPC resources and expertise in Computational Electro-magnetic

TRANSFER LEARNING 


\section{Summary}

- Research on applying DL techniques to multi-sensor information fusion is evolving

- Followings are key research that needs to be addressed:

- Filling the Gap/mismatch between measured and synthetic data

- Transfer Learning over operating spaces (range, resolution, target settings)

- Robust evaluation of the algorithms

- Operational deployment considerations, computation efficiency (SWaP-C)

[1] Big Data and Analytics. http://www.ibmbigdatahub.com/infographic/four-vs-big-data

[2] "MSTAR Public Dataset." The Sensor Data Management System. U.S. Air Force, 20 Sept. 2015. Web. 12 June 2016.

[3] W. Chapman, S. Ranka, S. Sahni, M. Schmalz, U. Majumder, "Parallel processing techniques for the processing of SAR data on GPUs", IEEE International Symposium on Signal Processing and Information Technology (ISSPIT), 2011.

[4] Convolution Neural Networks. www.wildml.com/2015/11/understanding-convolutionneural-networks-for-nlp

[5] Jia, Yangqing, et al. "Caffe: Convolutional architecture for fast feature embedding." Proceedings of the 22nd ACM international conference on Multimedia. ACM, 2014.

[6] S. Chen, H. Wang, F. Xu and Y. Q. Jin, "Target Classification Using the Deep Convolutional Networks for SAR Images," in IEEE Transactions on Geoscience and Remote Sensing, vol. 54, no. 8, pp. 4806-4817, Aug. 2016. 


\title{
Deep Learning for Object Recognition from High Volume Radio Frequency Data
}

\author{
Uttam K. Majumder ${ }^{\mathrm{a}, 1}$, Erik P. Blasch ${ }^{\mathrm{b}}$ \\ ${ }^{a}$ Air Force Research Laboratory, Information Directorate, 525 Brooks Road, Rome, NY13441. \\ ${ }^{\mathrm{b}}$ Air Force Office of Scientific Research, Wright-Patterson AFB, OH 45433
}

\begin{abstract}
Much research efforts have been devoted to applying deep learning (DL) algorithms in video imagery for object recognition. However, very limited publications can be found on technical challenges and approaches to execute DL algorithms in radio frequency (RF) data. This talks highlights recent advancements of DL on synthetic aperture radar (SAR) imagery for object recognition. Radar enables imaging ground objects at far greater standoff distances than an electrooptic sensor. Further, radar enables imaging a scene and obtaining salient features of objects in all weather/day-night conditions. One example is that future self-driving/autonomous vehicles/cars could integrate radar among other sensors for decision making while sharing the roads and avoiding collisions/accidents. Existing non-DL based object recognition algorithms are less accurate and require impractically large computing resources. DL enables more accurate, realtime/non-realtime, and low-power object recognition system development. An examples is presented on Convolution Neural Network (CNN) based SAR object recognition for GPU and energy efficient computing systems. Results demonstrate acceptable classification accuracy on relevant SAR data.
\end{abstract}

Keywords: Deep Neural Network (DNN), Artificial Intelligence (AI), Synthetic Aperture Radar (SAR), Radio Frequency (RF), Big Data

\section{RF BIG DATA}

According to IBM[1, 2], big data has several characteristics. Among these are volume, velocity, variety, and veracity. A big volume of data could be processing terabytes to exabytes or more data in a milliseconds or seconds. In some applications, RF data could be collected from petabytes to exabytes and these data need to be processed (e.g., object recognition) in real-time or near real-time. Radar images are difficult for humans to analyze. It requires significant efforts for accurate interpretation; however a DL system is very capable to interpret these images. The benefits of utilizing radar technology are evident given its advantages over electro-optical imaging. Specifically, radar is able to operate in a variety of operating conditions-including poor visibility, inclement weather, and night-time settings. Given the disparity between the technical capabilities of radar and its difficulty of interpretation, it is a goal to develop accurate methods for automatically recognizing objects in radar images without the need for expensive expert analysis. The benefits of object recognition for radar imagery include developing self-driving cars to autonomous systems.

\footnotetext{
${ }^{1}$ Correspondence: ukmccny@gmail.com
} 
Though most recent work in applying artificial neural networks (ANN) to computer vision has focused on electro-optical images, the application to radar imagery is of particular interest for our research. Previously, achieving RF object classification using DL methods was the absence of necessary computational power. A radar imagery data collection project-called Moving and Stationary Target Acquisition and Recognition (MSTAR) program-was funded by DARPA and the AFRL in the 1990s to fill the void in available radar data for object classification research[3, 4]. Additionally, the recent introduction of affordable GPU computing resources[5] has made the efficient processing of datasets for deep learning (DL) applications a reality for RF object classification research efforts.

\section{CONVOLUTIONAL NEURAL NETWORKS}

Convolutional neural networks (CNN) are a special case of neural networks, as they make the explicit assumption that the input data are of constant size. This enables certain features to be encoded in them. In particular, the computational units, or "neurons," share parameters with their neighbors. The connections conceptually result in an intuitive visualization of the network as a series of filters that "slide" or "pool" over regions they are connected to-producing mathematical output based on the parameters, or "weights," of each filter. The output produced by these filters is then fed to other filters connected to them, and so on, concluding with a fully connected dens network shown in Figure 1.

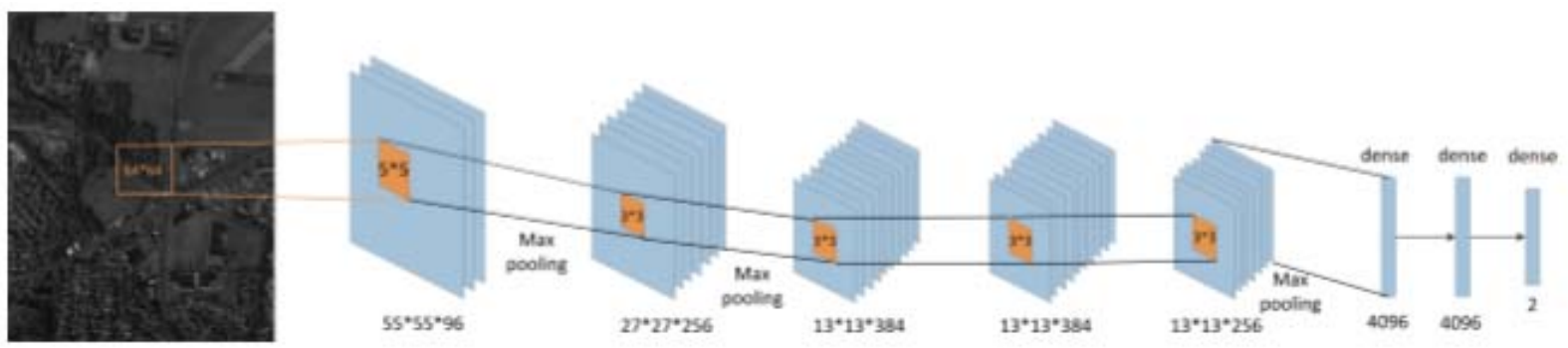

Figure 1: Illustration of Convolution Neural Networks [6]

Through training, these filters learn to respond to complex features in image data. Filters closer to the data input layer learn to recognize simple features, such as edges. Mid-level filters might learn compositions of edges, like simple shapes. High-level filters then learn complex abstractions of the data, such as compositions of shapes forming full objects of interest. Thus, CNNs are a natural fit for working with the conceptual hierarchy of features present within image data.

\section{EXPERIMENTS ON RF OBJECT CLASSIFICATIONS}

Software tools used for this research are: Caffe, DIGITS, Python, and LMDB. Caffe is a deeplearning framework maintained by the Berkeley Vision and Learning Center (BVLC)[7]. Caffe provides full end-to-end capability for deep learning tasks, and is the deep learning framework of choice for many in the machine learning community.

Experimental data were extracted from the MSTAR public dataset [3]. The MSTAR public dataset contains several hundred SAR images of a variety of targets collected in varying conditions. Sandia 
National Laboratory used an X-band radar sensor at 1-foot resolution in spotlight mode to collect target data at 15, 17, 30, and 45-degree depression angles. Images taken at varying azimuth angles provide a 360-degree view of each given target. The images are $128 \times 128$ pixel chips containing a target roughly centered within its background. Bulldozers, trucks tanks, and anti-aircraft vehicles are among the targets included in the dataset as shown in Figure 2 with the SAR images in Figure 3.

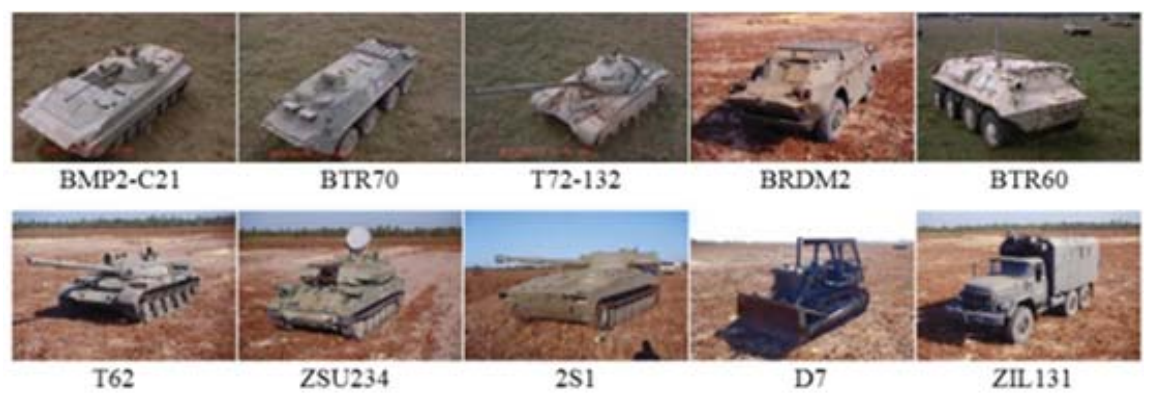

Figure 2: Electro-optic Images of Objects

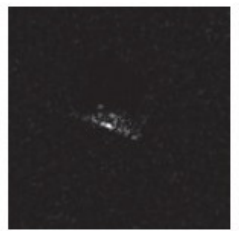

BMP2

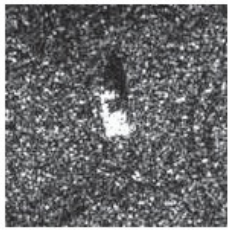

T62

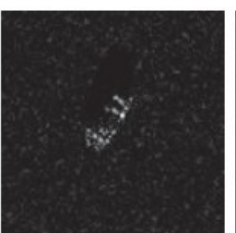

BTR70

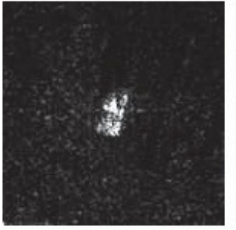

ZSU234

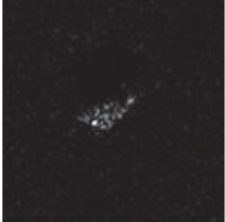

T72

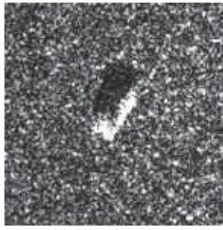

$2 S 1$

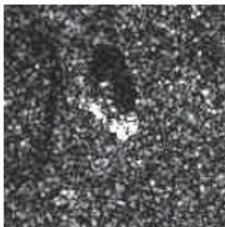

BRDM2

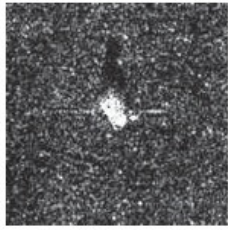

D7

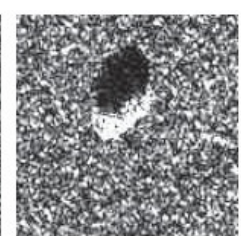

BTR60

Figure 3: SAR Images of Objects

\section{RESULTS}

On MSTAR public release data [3], 99\% classification accuracy was achieved using the model specifications described in Chen, et al. [8]. Most DL architectures tested reached an accuracy limit of $98.3 \%-98.7 \%$, depending on testing batch size and number of testing iterations ran. This held true for models employing small convolution filters, models with additional convolution layers before pooling, and models with fully connected layers at the end of the network architecture. Dropout regularization provided an accuracy improvement of roughly $0.5 \%$ over models not employing a DL technique. Though a relatively modest improvement, it is nonetheless indicative of the benefits of using dropout to ensure a model's ability to generalize to new data. 


\section{CONCLUSION AND FUTURE RESEARCH}

Research on applying DL techniques to multi-sensor information fusion is evolving, such as for image fusion [9]. Integrating RF sesnsor for Artifical Intelligence (AI) and information fusion has lots of technical challenges. Followings are key research that needs to be addressed:

- RF Synthetic Data Research: DL algorithms require lots of training data. Hence, research needs to be conducted to develop high fidelity synthetic RF data to augment measured RF data. This is important as measured RF data are expensive to collect.

- Transfer Learning Algorithms Development: Develop transfer learning techniques over operating spaces (e.g., range, resolution, target settings)

- Robust Evaluation of DL Algorithms: Results (classification accuracy) of DL algorithms are meaningless unless they are validated with representative operating environments (e.g., environment) [10]. Hence, validation and verification for reproducible object classification results in extensive operating conditions are very important.

- Computational Efficiency: Developing a real-time training algorithms and size, weight, and power-constrained (SWaP) computing systems will be required for future DL-based AI systems.

\section{REFERENCES}

[1] Big Data and Analytics. http://www.ibmbigdatahub.com/infographic/four-vs-big-data

[2] E. Blasch, M. L. Pugh, C. Sheaff, J. Raquepas, P. Rocci, "Big data for space situation awareness," Proc. SPIE, Vol. 10196, 2017.

[3] MSTAR Public Dataset." The Sensor Data Management System. U.S. Air Force, 20 Sept. 2015.

[4] E. Blasch, Derivation of a Belief Filter for Simultaneous High Range Resolution Radar Tracking and Identification, Ph.D. Thesis, Wright State University, 1999.

[5] W. Chapman, S. Ranka, S. Sahni, M. Schmalz, U. Majumder, "Parallel processing techniques for the processing of SAR data on GPUs," IEEE International Symposium on Signal Processing and Information Technology (ISSPIT), 2011.

[6] M. Yi, F. Yang, E. Blasch, C. Sheaff, K. Liu, G. Chen, H. Ling, "Vehicle classification in WAMI imagery using deep network," Proc. SPIE, Vol. 9838, 2016.

[7] Y. Jia, et al. "Caffe: Convolutional architecture for fast feature embedding." Proceedings of the 22nd ACM international conference on Multimedia. ACM, 2014.

[8] S. Chen, H. Wang, F. Xu and Y. Q. Jin, "Target Classification Using the Deep Convolutional Networks for SAR Images," in IEEE Transactions on Geoscience and Remote Sensing, vol. 54, no. 8, pp. 4806-4817, Aug. 2016.

[9] Y. Zheng. E. Blasch, Z. Liu, Multispectral Image Fusion and Colorization, SPIE, 2018.

[10] V. Kaufmann, T. Ross, E. Lavely, and E. Blasch, "Score-based SAR ATR Performance Model With Operating Condition Dependencies," Proc. of SPIE, Vol. 6568, 2007.

\section{DISCLAIMER}

The views expressed in this article are those of the author and do not reflect official policy of the United States Air Force, Department of Defense or the U.S. Government. 


\title{
Retrospectives on the Applications AI and Deep Learning in Information Fusion
}

\author{
Ivan Kadar \\ Interlink Systems Sciences, Inc. \\ 1979 Marcus Avenue, Lake Success, NY 11042
}

BACKGROUND-PERSPECTIVES

In this position paper concrete examples of the use of Neural Networks (NNs) and Artificial Intelligence (AI) components in Information Fusion (IF), are presented, based on work performed in the 1980s and 1990s-on at Grumman Aerospace Corporation, Advanced Technology Development Department, in Bethpage NY.

While the application of AI was prominent before NNs became popular, this paper starts with a description of my short verbal introduction to the subject at the panel, viz., NNs; and subsequently expands on the subject to depict the application of AI and NNs for IF applications.

As further detailed below, as part of the work at the Advanced Technology Development Department, of the Knowledge-Based Processing Systems Group and associated Knowledge-Based AI Technology development laboratory (1984-1990), founded and managed by the author starting in 1983, we used: tracking, association and fusion algorithms, and developed: e.g., Common-LISP-based algorithms/programs/systems, including an interactive digital LISP-based image processing facility for both low-level vision processing and for an initial capability of high-level image understanding towards target recognition; used Expert Systems Tools [1], NNs [2], Dempster-Shafer theory (DST)based algorithms for evidence representation and combination/fusion [3] and Fuzzy-Sets [4]; the latter three were also considered part of AI and not IF during that time frame. Given the available tool sets, we also developed the architecture, designed and implemented the simulation of a Tactical Assessment Expert System (TAES) [5]; all delineated in the sections below.

\section{NEURAL NETWORKS}

During my short introduction at the panel discussion the subject being addressed and the participants, only highlighted one aspect of the subject: "Deep Learning" [6,7], viz., the use of Neural Networks (NNs) for significant feature(s) recognition back in the 1987-1990's-on.

During that time frame we used a three hidden layer abductive polynomial NN [8] as the feature selection component of a ground-based-emitter discrimination algorithm in mid 1990s, resulting in a US Patent in 1999 [9]. Other components of the algorithm included Fuzzy Sets [4], and a related classifier.

An example abductive polynomial $\mathrm{NN}$ is shown below [8]:

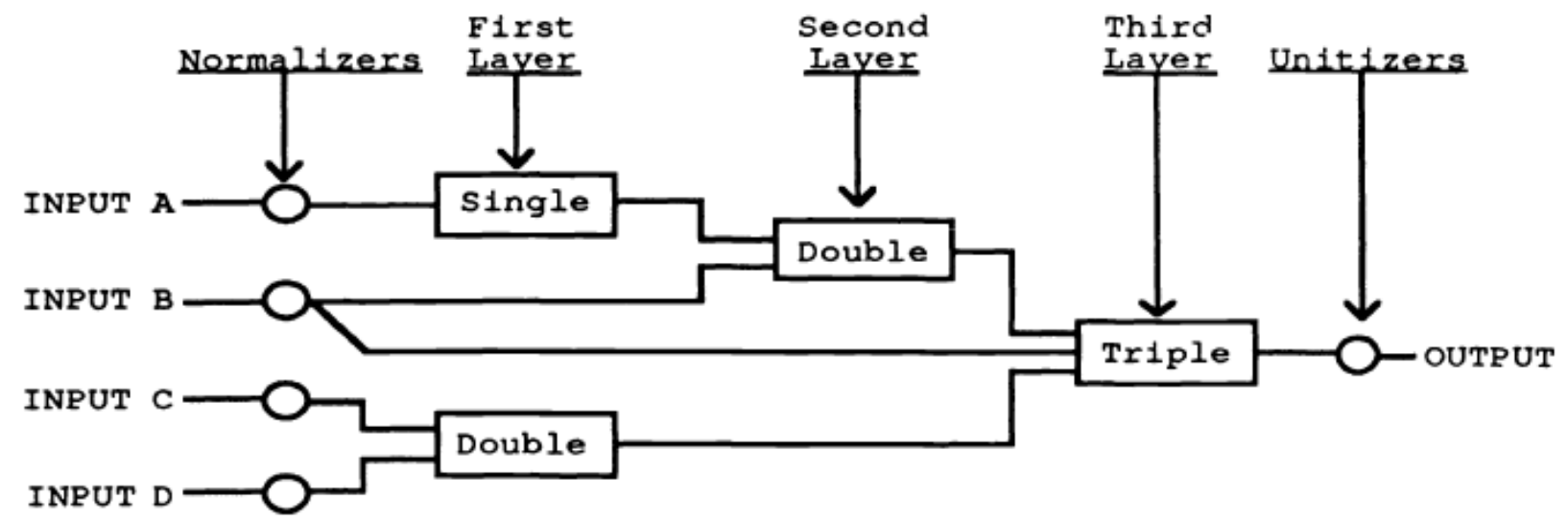

where, in the equations shown in the algebraic forms below, the w's are the weights learned by regression, and the x's are the input variables. 
Singles, doubles, and triples are elements whose names are based on the number of input variables. The algebraic form of each of these elements is shown in the following equations:

$$
\begin{aligned}
& \text { Single }=W 0+\left(W 1^{*} X_{1}\right)+\left(W 2^{*} X_{1}{ }^{2}\right)+\left(W 3^{*} X_{1}{ }^{3}\right) \\
& \text { Double }=W 0+\left(W 1^{*} X_{1}\right)+\left(W 2^{*} X_{2}\right)+\left(W 3^{*} X_{1}{ }^{2}\right)+\left(W 4^{*} X_{2}{ }^{2}\right)+\left(W 5^{*} X_{1}{ }^{*} X_{2}\right)+\left(W 6^{*} X_{1}{ }^{3}\right) \\
& +\left(W 7^{*} X_{2}{ }^{3}\right) \\
& \text { Triple }=W 0+\left(W 1^{*} X_{1}\right)+\left(W 2^{*} X_{2}\right)+\left(W 3^{*} X_{3}\right)+\left(W 4^{*} X_{1}{ }^{2}\right)+\left(W 5^{*} X_{2}{ }^{2}\right)+\left(W 6^{*} X_{3}{ }^{2}\right)+\left(W 7^{*} X_{1}^{*} X_{2}\right) \\
& +\left(W 8^{*} X_{1}^{*} X_{3}\right)+\left(W 9^{*} X_{2}^{*} X_{3}\right)+\left(W 10^{*} X_{1}^{*} X_{2}^{*} X_{3}\right)+\left(W 11^{*} X_{1}{ }^{3}\right)+\left(W 12^{*} X_{2}{ }^{3}\right)+\left(W 13^{*} X_{3}{ }^{3}\right)
\end{aligned}
$$

Figure 1. An abductive polynomial NN example [8]

Given the CPU clock speeds of the mid 1990s, the training alone took two days. That is, computers were very slow (100-300 MHz clock speeds), memory was scarce and very expensive, and even the three hidden layers network took a day to converge to the solution.

The term "Deep Learning" $[6,7]$ (referring to hidden layers in NNs) was not introduced at that time although the network had three hidden layers, and indeed it "deep" learned.

Of course, today's Deep Learning NNs [7, 10] have multitudes of hidden layers for features recognition, but even the fastest special-chip-based networks do not always converge in real-time use quickly enough, (as mentioned in articles published in the Technology Related Business Sections in the New York Times during the Spring 2018).

\section{ARTIFICIAL INTELIGENCE (INCLUDES NNs)}

Sections below, illustrate the complementary interactions and harmonious use of AI and NNs components in IF applications. Part of the material is adapted in part from the author's position paper within: "Results from Levels $2 / 3$ Fusion Implementations: Issues, Challenges, Retrospectives and Perspectives for the Future - An Annotated Perspective" presented at the SPIE Signal Processing, Sensor Fusion and Target Recognition XVII conference, Proc. SPIE Vol. 6968, Orlando Fl., April 2008.

It is well known that the concept of Situation Awareness (SA), (Level 2), and Threat Assessment (TA), (Level 3); SA/TA existed before the Joint Director of Laboratories Fusion Model (JDL) [11, 12, 13], viz., JDL established the numerical representation and "at-that-time" definition of Fusion "Levels" [9]. This is illustrated in a "circa 1984-1986 vintage" simulated "Tactical Assessment Expert System architecture," [14] depicted in Figure 2.

The TAES system utilized knowledge-based Expert Systems tools combined with numerical algorithms with the primary objective to reduce pilot workload so the pilot becomes the systems manager not just the operator.

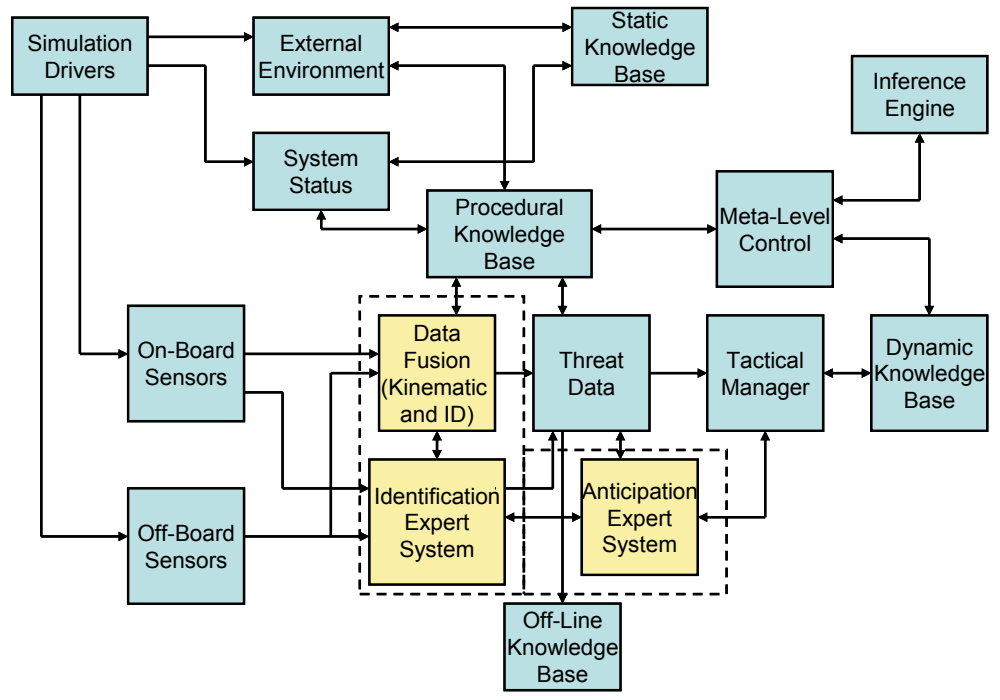

Figure 2. Tactical Assessment Expert System Functional Architecture

Ixxxvi 
That is, the TEAS system could inform the pilot: targets detections/IDs, environmental data and system status reports, and suggested tactical decisions for pilot's override. This was accomplished by modeling and encoding pilot's thought processes (via interviews) to form of an "experienced-software-copilot" during the SA/TA knowledge acquisition stage. The fundamental construct/design guidance for this system was based on an early fusion model (before JDL), called the "Perceptual Reasoning Machine (PRM) paradigm" [15], shown as an internal governing component of the Generic Information Fusion Process Model System (PMS) [14,15,16,17] shown in Figure 3. The dotted and yellow-highlighted blocks shown in Figure 2, correspond to the PRM functions shown in Figure 3. Figure 4 depicts the information flow among the PRM elements.

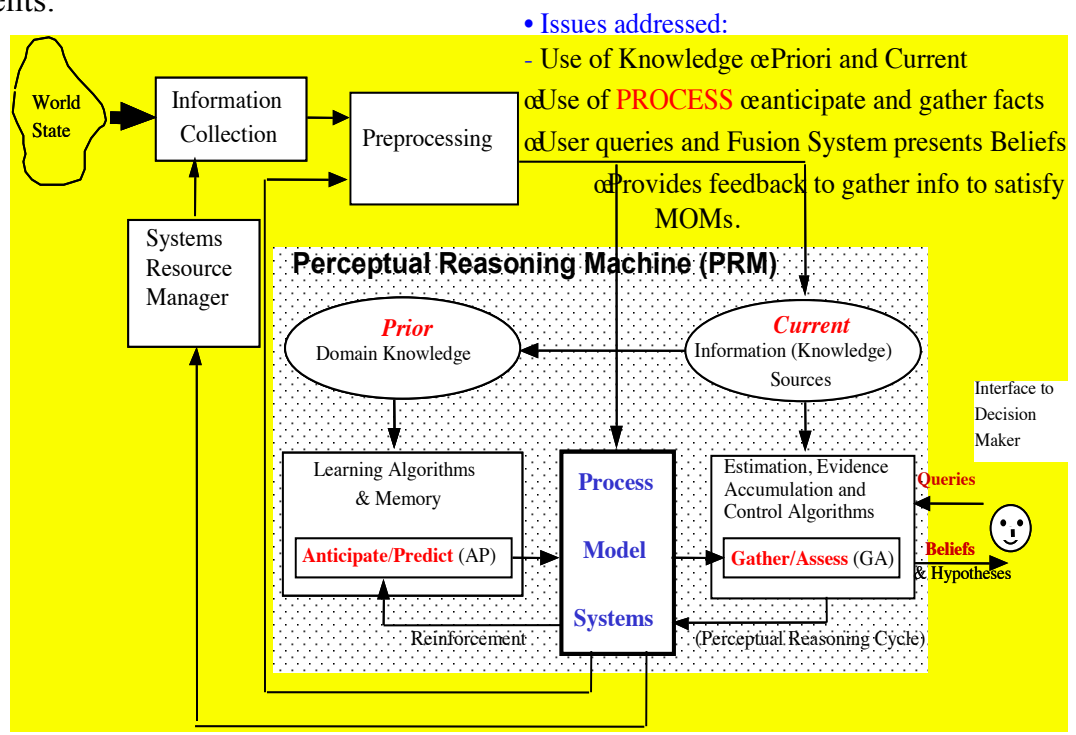

Figure 3. Genetic Information Fusion Process Model System (PMS)

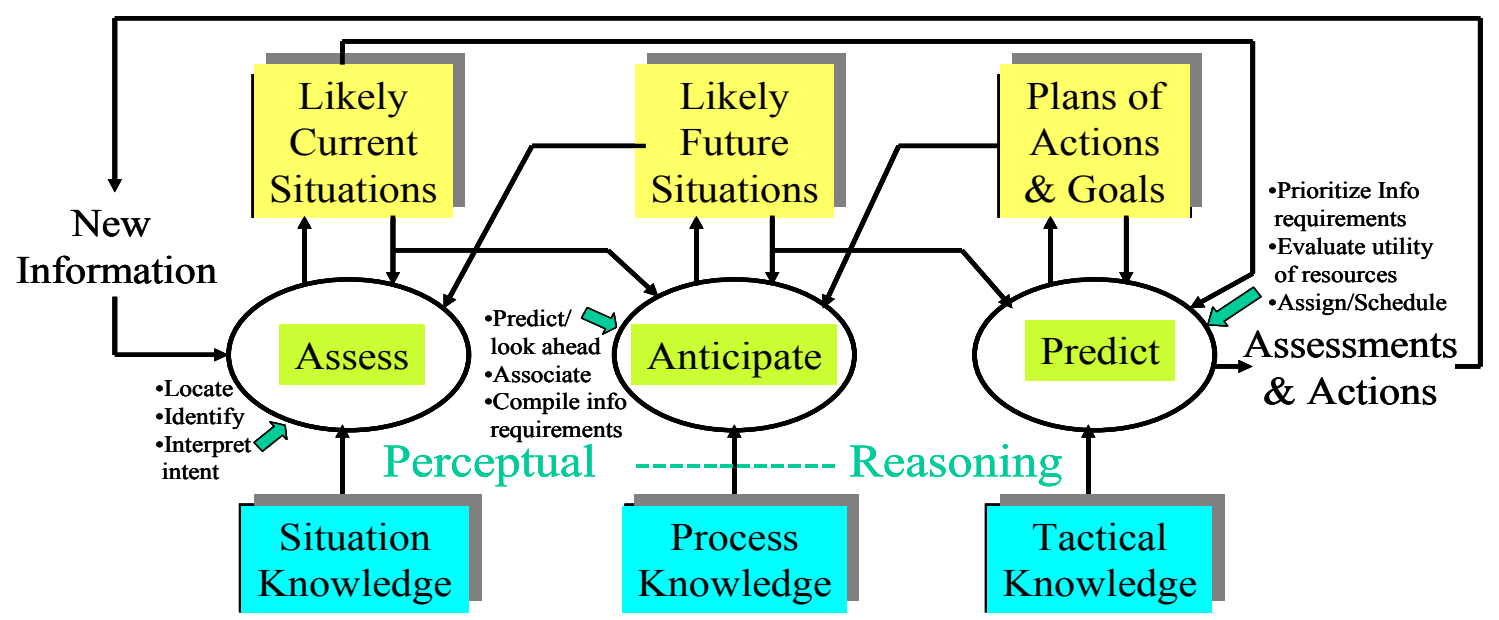

The "assess/(Gather)" module responding The "assess" module provides likely current situations to "dynamically managed" received multisource information to the "predict"/(preplan/act) module, which information uses additional information from its KB and from the "anticipate" module to form a database of likely current situations which include potential detected threats. along with its KB and likely future situations information from the "anticipate" module, issues assessments, identifies potential threats, provides plans of actions and goals, and as needed, request actions for additional information to confirm or The "anticipate" module provides information on expected likely future situations" for short and long-duration planning based on likely
current situations from the "assess" module; prior, learned, process and tactical knowledge, and associated likely hypotheses.

Figure 4. Information Flow among PRM Elements [Note: $\mathbf{K B}=$ Knowledge Base] 
The PRM construct, depicted in Figures 3 and 4, can be viewed as a "meta-level information management system", in general, and specifically when used within PMS, which is, a set of procedures and algorithms that capture the functional (temporal and/or spatial) dependency relationships of the task or processes being modeled [14-17]. PRM consists of a feedback planning/resource control system whose interacting elements are: "gather/assess", "anticipate" and "predict" [15-17], which are detailed, along with the required knowledge-bases, in Figure 4. Note: the "gather" part of the "gather/assess" module (shown in Figures 3 and 4) fuses optimally weighted information from multiple sensors/sources, and "assess" part functions are depicted in Figure 4.

\subsection{Evolving tools for Levels $2 / 3$}

The TEAS software architecture, shown in Figure 2, was built on Common LISP and using the Automated Reasoning Tool (ART) [18] an expert system building tool. There were several knowledge bases (KBs) as shown in Figure 2, (1) Declarative KB (functional - not shown) consisting of: Static KB- relations via semantic network using inheritance wherein the system exploits the inheritance structure of the KB to interpret incomplete data and a Pop-up menu driven on-Line KB to keep track of targets encountered, and to support interpretation of incomplete data; and, (2) Procedural/Operational KB consisting of: Production rules, interacting with a Dynamic KB using mathematical constructs - associated with the Control KB which are coupled with the interacting feedback structure of the PRM components of: Dempster-Shafer evidential reasoning (part of ID fusion expert system), Data Fusion/Tracking and Anticipation Expert Systems providing the reasoning mechanism for TEAS to arrive at a comprehensive interpretation of uncertain situations. The TEAS system was totally data Driven, i.e., LIFO - rules groups fired independently based on available data, allowing all modules to access to information during any stage of the program. Simulation results illustrated the interaction between a hypothetical scenario pilot thought process model (database derived from Jane's Book in all the Worlds Aircrafts) and the system, using simulated sensor reports to handle uncertainty. The TEAS system ran on the Symbolics 3675 LISP machine. The question arises how would one implement the software architecture of TAES today and what has changed since it was built.

As evident from the TAES construct, early approaches to higher-level fusion evolved from the mainstream use of early expert system tools (e.g., Knowledge Engineering Environment, "KEE” [19], Automatic Reasoning Tool, "ART” [18] built in Common-Lisp, both rule-based providing forward and backwards chaining, while ART provided hypotheses generation capability and prediction), other tools were based on: strings oriented symbolic (objects-oriented) language (SNOBOL-4) for pattern matching, common-LISP, logic (PROLOG), logical templates, procedural-LISP-based, such as Procedural Reasoning System "PRS", case-based languages, Blackboard (BB) system [20] representations, associative memory [21], schema-based languages and neural networks (NN) for knowledge elicitation/learning/acquisition (viz., background NNs learning the pilot's functions), evidential reasoning and ID declarations fusion using Dempster-Shafer calculi, tracking and related association algorithms, along with some of the basic methodologies remaining a part of current approaches. It should be noted: the "anticipate/predict" module of PRM (see Figure 4) was initially implemented using a KB of prior domain knowledge (which is automatically updated with current/latest knowledge), an inference engine and ART. Subsequently it was modified and used an associative memory [21] NN provided at that time by DEC corporation. The associative memory provided the "perceptual reasoning associative recall" function [22] in the PRM.

Current, and potential future trends, are primarily based on agent-based models [23] of interactions, including Blackboard (BB) systems [20], NN behavioral learning systems for knowledge acquisition, ontology representations (extending schemas), probability (Bayes-nets and Dempster-Shafer calculi and its extension [24, 25. 26] and possibility (fuzzy-sets)-based methods [27], graph theory oriented relational representations, game theoretic methods of optimization, some coupled with influence diagram formulations [28], but not excluding rule-based expert system tools, such as CLIPS built using $\mathrm{C}$ and JAVA [29], with the above representing a non-exhaustive representative list. The author is not aware of any comprehensive studies to compare the efficacy of the "historical main stream" and "currentmain stream" trends in order to learn from experience.

\subsection{Knowledge representation and reasoning (KKR) approaches/issues, when AI and NNs are available}

Related to section 2.1, methods of knowledge elicitation/acquisition, learning, representation and reasoning (KRR) have not appeared to have made significant strides over the past several years in spite of several conferences devoted to KRR [30], illustrating the difficulty associated with this topical area. The following list highlights potential KRR issues and challenges $[30,31]$ : 
- $\quad$ Adequacy of KRR

- Using logic, semantics, ontology, probabilistic methods, neural networks, associative memory, blackboard, simulations, rules and computation - how to quantify and measure?

- Expressiveness of models vs. tractability of inference

- Measures of richness of model vs. knowledge that inference is decidable and will produce and answer efficiently; and why correct and how arrived at that answer? [31]

- Managing Complexity

- Limits about tractability - how to bound the problem with incomplete knowledge

- Data Information

- How to manage heterogeneous and uncertain Knowledge Sources, and detect duplicate or incomplete concepts

- Knowledge Acquisition/Elicitation Issues

- Expert's difficulty in verbalizing knowledge

- Reliability and uncertainty of knowledge, and how to calibrate (ground truth) - Methods for reasoning and discovery under uncertainty

- Indirect learning of knowledge - "on-line background" learning of "selected" features

- Presentation of knowledge to different users/experts with different levels of expertise. i.e., what is pragmatic?

\section{CONCLUSIONS}

The purpose of this position paper is to illustrate the 1987-1990's use of NNs "Deep Learning" and AI algorithms, and subsequently highlight the 1984-1987-on retrospectives and perspectives on issues and challenges of Levels 2/3 information fusion using $\mathrm{AI}$ and NNs methods as components of the implementations, by presenting an independent point of view. There are many other possible additional implementation issues and challenges remaining, for example, in: model refinement, computational and processing methods, optimization, automation and decision making under uncertainty, human-machine interface and integration, distributed systems, knowledge elicitation, deep learning and representation, and potentially many more issues and challenges that hopefully will be addressed as part of future research in this area using new approaches.

\section{REFERENCES}

[1] F. Hayes-Roth et al. (Eds.) Building Expert Systems, Addison Wesley Pub. Co., Reading, MA 1983.

[2] R. L. Barron, et al, "Adaptive Learning Networks: Development and Application in the U. S. of

Algorithms Related to GMDH," Self-Organizing Methods in Modeling: GMDH Type Algorithms,

ed. by S. J. Farlow, Marcel-Dekker, Inc., New York, 1984.

[3] P. Shenoy and G. Shafer, "Propagating Belief Functions with Local Computations", IEEE Expert, pp. 43-52,Ik1986.

[4] L.A. Zadeh, "Fuzzy Sets", Information and Control, Vol. 8, pp.338-353, 1965

[5] I. Kadar and E. Baron-Vartian, "Process Modeling: A Situation Assessment Expert System", Proceedings AIAA

Computers in Aerospace Conference, Wakefield, MA, October 1987.

[6] https://en.wikipedia.org/wiki/Deep learning

[7] Y. LeCun, Y.Bengio and G. Hinton “ Deep learning”, Nature, 521(7553), 436-444, 2015

[8] G. J. Montgomery, K. C. Drake, "Abductive networks," Proc. SPIE1294, Applications of Artificial Neural Networks, 1 August 1990.

[9] I. Kadar et al. "System and Method for Functional Recognition of Emitters" US Patent March 16, 1999

[10] S. Haykin, Neural Networks and Learning Methods, $3^{\text {rd }}$ Ed. Prentice Hall, 2008

[11] F. White, "Data Fusion Lexicon", Joint Directors of Laboratories, Technical Panel for C3, Data Fusion SubPanel, Naval Ocean Systems Center, San Diego 1987

[12] A. Steinberg, C. Bowman and F. White, "Revisions of the JDL Fusion Levels", Proc. of the SPIE Sensor Fusion

Architectures, Algorithms and Applications III Conference, pp.430-441, April 1999.

[13] J. Llinas, C. Bowman, G. Rogova., A. N. Steinberg, E. Waltz, F. White, "Revisiting the JDL Data Fusion Model II,

Proc. of the 7th International Conference on Information Fusion, June 2004, Stockholm, Sweden 
[14] I. Kadar and E. Baron-Vartian, "Process Modeling: A Situation Assessment Expert System”, Proc. AIAA Computers in Aerospace Conf. Wakefield, MA, October 1987.

[15] I. Kadar, "Data Fusion by Perceptual Reasoning and Prediction", Proceedings 1987 Tn-Service Data Fusion Symposium, Johns Hopkins University Applied Physics Laboratory, Laurel, MD, June 1987

[16] I. Kadar, "Perceptual Reasoning Managed Situation Assessment and Adaptive Fusion Processing", Proc. Signal

Processing, Sensor Fusion \& Target Recognition X, Ivan Kadar Editor, Proc. SPIE Vol. 4380, April 2001.

[17] I. Kadar, "Perceptual Reasoning in Adaptive Fusion Processing", Proceedings Signal; Processing, Sensor Fusion and Target Recognition XI, Ivan Kadar Editor, Proc. SPIE Vol. 4729, April 2002.

[18] https://pvhaley.wordpress.com/2008/02/20/haley-art-syntax-lives-on-in-open-source-java-rules/

[19] https://en.wikipedia.org/wiki/Knowledge_Engineering_Environment

[20] https://en.wikipedia.org/wiki/Blackboard_system

[21] T. Kohonen, Self Organization and Associative Memory, Springer Verlag, New York 1984.

[22] I. Kadar, "Knowledge Representation Issues in Perceptual Reasoning Managed Situation Assessment", Invited Panel Session on "Issues and Challenges of Knowledge Representation and Reasoning in Situation Assessment (Level 2 Fusion), Organizer: Ivan Kadar; Moderators: Ivan Kadar and James Llinas; Proceedings of the $8^{\text {th }}$ International Conference on Information Fusion, July 25-29, 2005, Philadelphia, PA

[23] J. M. Bradshaw, Software Agents, MIT Press 1997.

[24] J. Pearl, Probabilistic Reasoning in Intelligent Systems: Networks of Plausible inference, Morgan Kauffman Publishers, Inc., San Mateo, CA 1988.

[25] G. Shafer, The Mathematical Theory of Evidence, Princeton University Press, Princeton New Jersey 1976

[26] Dezert-Smarandache Theory of Plausible, Paradoxist, and Neutrosophic Reasoning.mht

[27] G. Klir, Uncertainty and Information: Foundations of Generalized Information Theory, John Wiley, 2006

[28] D. Koller and B. Milch, "Multi-Agent Influence Diagrams for Representing and Solving Games", Games and Economic Behavior, Vol. 45, No.1, 2003.

[29] www.ghg.net/clips/CLIPS.html A Tool for Building Expert Systems

[30] www.kr.org - The Principles of Knowledge Representation and Reasoning (KRR)

[31] D. Gunning, "Explainable Artificial Intelligence (XAI)" DARPA-BAA-16-53, August 10, 2016 